\title{
On BGG resolutions of unitary modules for quiver Hecke and Cherednik algebras
}

\author{
C. Bowman ${ }^{1} \cdot$ E. Norton ${ }^{2}$. J. Simental ${ }^{3}$
}

Accepted: 12 November 2021 / Published online: 4 January 2022

(c) The Author(s) 2022

\begin{abstract}
We provide a homological construction of unitary simple modules of Cherednik and Hecke algebras of type $A$ via BGG resolutions, solving a conjecture of BerkeschGriffeth-Sam. We vastly generalize the conjecture and its solution to cyclotomic Cherednik and Hecke algebras over arbitrary ground fields, and calculate the Betti numbers and Castelnuovo-Mumford regularity of certain symmetric linear subspace arrangements.
\end{abstract}

Mathematics Subject Classification 16 S99 $\cdot 20 \mathrm{C} 20 \cdot 20 \mathrm{C} 08 \cdot 14 \mathrm{~N} 20$

\section{Introduction}

In [1], Bernstein-Gelfand-Gelfand utilise resolutions of simple modules by Verma modules to prove certain beautiful properties of finite-dimensional Lie algebras. Such resolutions (now known as BGG resolutions) have had spectacular applications in the study of the Laplacian on Euclidean space [19], complex representation theory and homology of Kac-Moody algebras [28], statistical mechanics and conformal field theories $[27,46,47]$, and they provide graded free resolutions (in the sense of commutative algebra) for determinantal varieties [20,40]. Remarkably, such resolutions have never

$凶 \quad$ J. Simental

jose@mpim-bonn.mpg.de

C. Bowman

Chris.Bowman-Scargill@york.ac.uk

E. Norton

E.Norton@kent.ac.uk

1 Department of Mathematics, University of York, Heslington, York YO10 5DD, UK

2 School of Mathematics, Statistics and Actuarial Science, University of Kent, Canterbury CT2 7FS, UK

3 Max-Planck-Institut für Mathematik, Vivatsgasse 7, 53111 Bonn, Germany

Birkhäuser 
been used in the study of symmetric and general linear groups in positive characteristic — or indeed anywhere in modular representation theory!

One of the most important problems in Lie theory is to classify and construct unitary simple representations. For Lie groups, this ongoing project draws on techniques from Dirac cohomology [35], Kazhdan-Lusztig theory [64], and the Langlands Program [58], and has provided profound insights into relativistic quantum mechanics [61]. The Cherednik algebra of a complex reflection group, $W$, is an important Lie theoretic object which possesses hallmarks from the classical theory: a triangular decomposition and a category $\mathcal{O}$ with a highest weight theory [29], analogues of translation functors [42], induction and restriction functors [3] with associated Harish-Chandra series [43], and Kazhdan-Lusztig theory [52] (for $W=G(\ell, 1, n)$ ). Both the unitary representations of real reductive groups [31-33] and those of Cherednik algebras [13] are of great importance in algebraic harmonic analysis.

Resolutions and cohomology of Cherednik algebras. For Cherednik algebras of symmetric groups, $H_{1 / e}\left(\mathfrak{S}_{n}\right)$, the simple unitary representations $L(\lambda)$ of $H_{1 / e}\left(\mathfrak{S}_{n}\right)$ were classified by Etingof, Griffeth and Stoica [23] by a combinatorial condition on the partition $\lambda$ of $n$ labeling the "highest weight" of $L(\lambda)$. In the spirit of classical results in Lie theory, Berkesch, Griffeth, and Sam subsequently conjectured that any unitary simple $L(\lambda)$ admits a BGG resolution [65, Conjecture 4.5]. The primary purpose of this paper is to prove Berkesch-Griffeth-Sam's conjecture and thus homologically construct the unitary simple $H_{1 / e}\left(\mathfrak{S}_{n}\right)$-modules. To state our main result, let us recall that the category $\mathcal{O}$ of the algebra $H_{1 / e}\left(\mathfrak{S}_{n}\right)$ is a highest weight category, with simple modules $L(\lambda)$ and standard modules $\Delta(\lambda)$, see Sect. 5.8 for precise definitions. We define a length function, $\ell: W \rightarrow \mathbb{N}$, on partitions in terms of the action of an affine Weyl group in Subsection 2.1.

Theorem A Associated to any simple unitary $H_{1 / e}\left(\mathfrak{S}_{n}\right)$-module, $L(\lambda)$, we have a complex $\mathbf{C}_{\bullet}(\lambda)=\bigoplus_{\lambda \triangleq_{v}} \Delta(\nu)[\ell(\nu)]$ with differential given by an alternating sum over all "one-column homomorphisms". This complex is exact except in degree zero, where $H_{0}\left(\mathbf{C}_{\bullet}(\lambda)\right)=L(\lambda)$.

Geometric resolutions. In contrast to classical papers on BGG resolutions and unitary representations, which usually (but not always! [12]) employ ideas from algebraic geometry, our methods are completely algebraic and moreover yield several geometric results. Namely, each standard module $\Delta(v)$ is a free $\mathbb{C}\left[x_{1}, \ldots, x_{n}\right]$-module, and as a consequence we obtain $\mathfrak{S}_{n}$-equivariant, graded free resolutions (in the sense of commutative algebra) for the $e$-equals variety

$$
X_{e, 1, n}:=\mathfrak{S}_{n}\left\{\left(z_{1}, \ldots, z_{n}\right) \in \mathbb{C}^{n}: z_{1}=\cdots=z_{e}\right\},
$$

and for the following algebraic varieties when $n=k e$ :

$$
X_{e, k, n}:=\mathfrak{S}_{n}\left\{\left(z_{1}, \ldots, z_{n}\right) \in \mathbb{C}^{n}: z_{i e+1}=\cdots=z_{(i+1) e} \text { for } 0 \leq i<k\right\} .
$$

We hence provide, see Propositions 8.2 and 8.6, formulae for the graded Betti numbers and calculate the Castelnuovo-Mumford regularity of these varieties - a notoriously 
difficult problem in general [16,57]. Moreover, we also provide formulae for these invariants in the cyclotomic case, where the equalities in the equations defining the above varieties become equalities up to multiplication by an $\ell$ th root of unity. Finally, we remark that the Cherednik algebra approach to geometric resolutions was inspired by the Lie theoretic construction of Lascoux's resolutions of determinantal varieties (via parabolic BGG resolutions of unitary modules) [20,65]; it would be interesting to find a purely geometric proof of the resolutions of our varieties by analogy with [40].

Motivation for the existence of BGG resolutions of unitary modules. Our proof of Theorem A only makes use of the combinatorial condition on the partition $\lambda$ labeling unitary representations [23], and does not make use of the concept of unitarity itself. Thus, although this section is not strictly necessary for the rest of the paper, we take a moment to explain, beyond the analogy with classical Lie theory, why it is reasonable to expect that a BGG resolution of unitary modules should exist. We remark, first, that the concept of a BGG resolution is a highest-weight-Morita-invariant homological construction, while the unitarity concept arises in harmonic analysis and it is not preserved by Morita equivalence. Up to highest-weight equivalence, the category $\mathcal{O}$ for the Cherednik algebra $H_{a / e}\left(\mathfrak{S}_{n}\right)$ only depends on the denominator, $e$, of the parameter whereas the module $L_{a / e}(\lambda)$ being unitary really depends on the numerator $a$, with the most general case being $a= \pm 1$. Thus, in order to connect BGG resolutions and unitary modules in a meaningful way, we first find a representation-theoretic concept at the level of categories and combinatorics that is equivalent to being unitary for the parameter $\pm 1 / e$.

Assuming $L(\lambda)$ has full support, we can achieve this using the (quiver) Hecke algebra. The representation theory of the algebra $H_{1 / e}\left(\mathfrak{S}_{n}\right)$ is closely connected with that of the Hecke algebra $\mathcal{H}_{q}\left(\mathfrak{S}_{n}\right)$ of the symmetric group at the root of unity $q=$ $\exp (2 \pi \sqrt{-1} / e)$; namely, there exists an exact functor $K Z: \mathcal{O}_{1 / e}\left(\mathfrak{S}_{n}\right) \rightarrow \mathcal{H}_{q}\left(\mathfrak{S}_{n}\right)$ which preserves (simple) unitary representations. Comparing results of Stoica [54] and Ruff [53] we can see that, for $\mathcal{H}_{q}\left(\mathfrak{S}_{n}\right)$ the class of unitary modules coincides with the much-studied calibrated/completely splittable modules (those on which the Jucys-Murphy subalgebra acts semisimply [37,49,53]) or equivalently the class of modules which are homogenous in the KLR grading on $\mathcal{H}_{q}\left(\mathfrak{S}_{n}\right)$. Thus, for fully supported $L(\lambda)$ we replace the condition " $L(\lambda)$ is unitary" with the equivalent condition that " $\mathrm{KZ}(L(\lambda))$ is calibrated/homogenous" and we construct a resolution of $\mathrm{KZ}(L(\lambda))$ by cell modules of $\mathcal{H}_{q}\left(\mathfrak{S}_{n}\right)$, which we also call a BGG resolution. This resolution upgrades a character formula from [53] to the categorical level. After this, we will lift the resolution back to a BGG resolution of $L(\lambda)$.

Modular BGG resolutions for symmetric groups and KLR algebras. A key ingredient to our proofs is to work in the 2-categorical setting of diagrammatic Cherednik algebras (or weighted KLR algebras) of [59]. The diagrammatic calculus is easier for calculation and benefits from a graded structure. The diagrammatic approach allows us to generalize the original conjecture to higher levels and arbitrary ground fields; we prove this more general version. We recast the combinatorial condition in type $A$ for $L(\lambda)$ to be unitary [23] as, the partition $\lambda$ lies in the fundamental alcove of the dominant chamber in an affine type $A$ alcove geometry. In our BGG resolution, $\Delta(v)$ appears in homological degree $d$ if and only if $v$ is obtained from $\lambda$ by reflecting across $d$ walls 
(increasing the distance from the fundamental alcove by 1 at each step). This alcove model vastly generalizes to the set of all $\ell$-partitions whose components each have at most $h$ columns, $\mathscr{P}_{n}^{\ell}(h)$. For any multipartition lying in the fundamental alcove we then construct a BGG resolution of the corresponding simple $H_{c}(G(\ell, 1, n))$-module. We remark that Griffeth has obtained a combinatorial description of the $\ell$-partitions that label unitary irreducible modules for $H_{c}(G(\ell, 1, n))$, [30], and it would be interesting to compare this condition to the one arising from the alcove model.

Working with quiver Hecke algebras furthermore allows us to obtain our results over an arbitrary field, $\mathbb{k}$. The search for an effective description of the dimensions of simple representations of symmetric groups over arbitrary fields is a centre of gravity for much research in modular Lie theory $[44,45,50]$. We show that our resolutions for unitary simples remain stable under reduction modulo $p$ - in other words the beautiful properties of unitary modules extend beyond the confines of characteristic zero (a necessary condition for the definition of unitary modules via bilinear forms to make sense) to for arbitrary fields. Finally, in Theorem 7.4 and Proposition 7.2 we obtain a simple closed form for the Mullineux involution, M, on unitary/homogenous simples and explicitly construct this isomorphism - to our knowledge, this is the first time such an isomorphism has been explicitly constructed (outside of the semisimple case).

This pivots the impact of our result from Cherednik algebras and geometry of subspace arrangements, to modular representations of the symmetric group. As our main result is the first of its kind for symmetric groups (providing a resolution of a given homogenous simple $D_{n}(\lambda)$ in terms of the grading-shifted Specht modules $S_{n}(v)$ for $\lambda \unrhd v$, see Subsection 1.2 for precise definitions) we state it now in this simplified form. For the far more general statement concerning cyclotomic quiver Hecke algebras, see Theorems 4.2 and 4.3.

Theorem B Let $\mathbb{k}$ be a field of characteristic $p>0$. For $D_{n}(\lambda)$ a homogenous simple (in the KLR grading) we have an associated $\mathbb{k} \mathfrak{S}_{n}$-complex $C_{\bullet}(\lambda)=$ $\bigoplus_{\lambda \unrhd_{v}} S_{n}(v)\langle\ell(\nu)\rangle$ with differential given by an alternating sum over all "one-column homomorphisms". This complex is exact except in degree zero, where

$$
H_{0}\left(C_{\bullet}(\lambda)\right)=D_{n}(\lambda)
$$

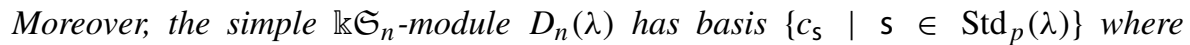
$\operatorname{Std}_{p}(\lambda) \subseteq \operatorname{Std}(\lambda)$ is the set of $p$-restricted tableaux. The action on this basis is given in Theorem 4.3. We have that $D_{n}(\lambda) \otimes \mathrm{sgn} \cong D_{n}\left(\lambda_{\mathrm{M}}\right)$ under the map $: c_{\mathrm{S}} \mapsto c_{\mathrm{S}_{\mathrm{M}}}$.

We thus provide the first instances of BGG resolutions anywhere in modular representation theory of finite and algebraic groups; in particular the first homological construction of a family of simple modules for symmetric groups. For Hecke algebras of type $B$, the simplest examples of our resolutions have appeared in work of mathematical physicists concerning Virasoro and blob algebras [27,46,47] and in Brundan-Stroppel's founding work on categorical representation theory $[11,12]$.

The bases of Theorem B first appeared in work of Kleshchev and Ram [37]. We remark that our results for the Hecke algebras depend only on the quantum parameter $e \in \mathbb{N}$ and are entirely independent of the characteristic of the underlying field (for $\ell=1$ we set $e=p$ in Theorems 4.2 and 4.3 to obtain Theorem B for symmetric groups). 
The partitions and multipartitions we consider (namely those lying in the fundamental alcove) have no restriction on their $e$-weight; calculating the composition series of the corresponding Specht modules for symmetric groups and Hecke algebras in positive characteristic is far beyond the current realms of conjecture (which at present have been stretched as far as $w(\lambda)<p^{3}$ for $h=3$ by Lusztig-Williamson [44]). Over $\mathbb{C}$, calculating the composition series of these Specht modules is theoretically possible using Kazhdan-Lusztig theory - however it quickly becomes computationally impossible - we provide examples of series of Specht modules (of rank $n$ as $n \rightarrow \infty$ ) for which the length of the composition series tends to infinity. Thus Theorem B provides the only contexts in which we can hope to understand unitary simple modules (see Sect. 4).

Structure of the paper. To finish the introduction, let us discuss the structure of the paper and of the proofs of Theorems A and B. We in fact deduce Theorem A from Theorem B. To that end, in Sect. 1 we introduce the quiver Hecke and diagrammatic Cherednik algebras. In Sect. 2 we recall the alcove geometric approach to these diagrammatic algebras, this will provide the combinatorics for proving Theorem B. In Sect. 3 we lift this combinatorics to the level of homomorphisms between standard modules, thus providing the skeleton of the resolutions in Theorem B; we also study the compositions and restrictions of these homomorphisms. In Sect. 4 we prove Theorem B, see Theorems 4.2 and 4.3 .

In Sect. 5, we pivot to rational Cherednik algebras. We first translate the path model of Sect. 2 into the language of abacus combinatorics used in formulating [65, Conjecture 4.5]. We then recall the connection between rational Cherednik algebras and the diagrammatic algebras of Sect. 1; this allows us to use Theorem B to prove almost all cases of Theorem A (see Theorem 5.24). In Sect. 6, we complete the proof of Theorem A and we also construct BGG resolutions of certain non-unitary representations. In Sect. 7 we give a simple formula, in terms of abacus combinatorics, to compute the Mullineux map on unitary representations. Finally, in Sect. 8 we explore the commutative algebra consequences of Theorem A .

\section{Diagrammatic algebras}

\subsection{Combinatorics}

Fix a charge $\left(e ; \kappa_{0}, \ldots, \kappa_{\ell-1}\right) \in \mathbb{N} \times \mathbb{Z}^{\ell}$. We now recall the diagrammatic Cherednik/weighted Hecke algebras of [59] and results concerning their representation theory from [6-8]. We define a partition, $\lambda$, of $n$ to be a finite weakly decreasing sequence of non-negative integers $\left(\lambda_{1}, \lambda_{2}, \ldots\right)$ whose sum, $|\lambda|=\lambda_{1}+\lambda_{2}+\ldots$, equals $n$. An $\ell$-partition $\lambda=\left(\lambda^{(0)}, \ldots, \lambda^{(\ell-1)}\right)$ of $n$ is an $\ell$-tuple of partitions such that $\left|\lambda^{(0)}\right|+\cdots+\left|\lambda^{(\ell-1)}\right|=n$. We will denote the set of $\ell$-partitions of $n$ by $\mathscr{P}_{n}^{\ell}$. Given $\lambda=\left(\lambda^{(0)}, \lambda^{(1)}, \ldots, \lambda^{(\ell-1)}\right) \in \mathscr{P}_{n}^{\ell}$, the Young diagram of $\lambda$ is the set of nodes,

$$
\left\{(r, c, m) \mid 1 \leq c \leq \lambda_{r}^{(m)}\right\}
$$


We refer to a node $(r, c, m)$ as being in the $r$ th row and $c$ th column of the $m$ th component of $\lambda$. Given a box, $(r, c, m)$, we define the content of this box to be $\operatorname{ct}(r, c, m)=\kappa_{m}+c-r$ and we define its residue to be $\operatorname{res}(r, c, m) \equiv \operatorname{ct}(r, c, m)$ $(\bmod e)$. We refer to a box of residue $i \in \mathbb{Z} / e \mathbb{Z}$ as an $i$-box. We set $\operatorname{res}(\lambda):=$ $\cup_{(r, c, m) \in \lambda} \operatorname{res}(r, c, m)$.

Remark 1.1 We wish to emphasise here that the notion of content is heavily dependent on the choice of charge. Two distinct charges will often result in the same residues and will give isomorphic Hecke algebras; however the resulting Cherednik algebras need not be isomorphic or even Morita equivalent, see [5]. However, they are derived equivalent, although we will not need this in this paper, [42,51].

Given $\lambda \in \mathscr{P}_{n}^{\ell}$, we let $\operatorname{Rem}(\lambda)$ (respectively $\operatorname{Add}(\lambda)$ ) denote the set of all removable (respectively addable) boxes of the Young diagram of $\lambda$, i.e. those boxes $(r, c, m) \in \lambda$ (resp. $(r, c, m) \notin \lambda)$ so that $\lambda \backslash\{(r, c, m)\}$ (resp. $\lambda \cup\{(r, c, m)\}$ ) forms the Young diagram of an $\ell$-partition. Given $i \in \mathbb{Z} / e \mathbb{Z}$, we let $\operatorname{Rem}_{i}(\lambda) \subseteq \operatorname{Rem}(\lambda)$ and $\operatorname{Add}_{i}(\lambda) \subseteq$ $\operatorname{Add}(\lambda)$ denote the subsets of boxes of residue $i \in \mathbb{Z} / e \mathbb{Z}$.

Each charge gives rise to a different ordering on $\mathscr{P}_{n}^{\ell}$, and hence a different Fock space, a different Cherednik algebra, and a different weighted lens through which to study the quiver Hecke algebra [5]. These charged ordering on $\mathscr{P}_{n}^{\ell}$ are given as follows:

Definition 1.2 Given $\kappa \in \mathbb{Z}^{\ell}$ such that $0 \leq \kappa_{i}-\kappa_{i+1}<e$ we write $(r, c, m) \triangleleft_{\kappa}$ $\left(r^{\prime}, c^{\prime}, m^{\prime}\right)$ if $\operatorname{res}(r, c, m)=\operatorname{res}\left(r^{\prime}, c^{\prime}, m^{\prime}\right)$ and either

(i) $\operatorname{ct}(r, c, m)<\operatorname{ct}\left(r^{\prime}, c^{\prime}, m^{\prime}\right)$ or

(ii) $\operatorname{ct}(r, c, m)=\operatorname{ct}\left(r^{\prime}, c^{\prime}, m^{\prime}\right)$ and $m>m^{\prime}$

For $\lambda, \mu \in \mathscr{P}_{n}^{\ell}$, we write $\mu \leq_{\kappa} \lambda$ if there is a bijective map $A:[\lambda] \rightarrow[\mu]$ such that either $A(r, c, m) \triangleleft_{\kappa}(r, c, m)$ or $A(r, c, m)=(r, c, m)$ for all $(r, c, m) \in \lambda$.

We reiterate that the orderings $\leq_{\kappa}$ and $\triangleleft_{\kappa}$ are heavily dependent on $\kappa \in \mathbb{Z}^{\ell}$.

Definition 1.3 Given $\lambda \in \mathscr{P}_{n}^{\ell}$, we define a tableau of shape $\lambda$ to be a map $\mathrm{t}:[\lambda] \rightarrow$ $\{1, \ldots, n\}$. We define a standard tableau to be a tableau in which the entries increase along the rows and columns of each component. We let $\operatorname{Std}(\lambda)$ denote the set of all standard tableaux of shape $\lambda \in \mathscr{P}_{n}^{\ell}$.

Definition 1.4 Given $\lambda \in \mathscr{P}_{n}^{\ell}$ we let $\mathrm{t}^{\lambda} \in \operatorname{Std}(\lambda)$ be the tableau obtained by placing the entry $n$ in the unique minimal removable box $(r, c, m) \in \lambda$ (under the $\leq_{\kappa}$ ordering) and then placing the entry $n-1$ in the unique minimal removable box of $\lambda \backslash\{(r, c, m)\}$ and continuing in this fashion.

We again emphasise that the definition of $\mathrm{t}^{\lambda}$ is highly dependent on the fixed choice of charge.

Definition 1.5 For $h \geq 0$ we say that $\kappa \in \mathbb{Z}^{\ell}$ is $h$-admissible if $h<\left|\kappa_{i}-\kappa_{j}\right|<e-h$ for $0 \leq i \neq j<\ell$. 
Remark 1.6 For $h \geq 0$ and $\kappa \in \mathbb{Z}^{\ell} h$-admissible the ordering $\triangleleft_{\kappa}$ in Definition 1.2 is a coarsening of the usual $c$-function ordering on the Fock spaces of Foda-LeclercOkado-Thibon-Welsh [5,59]. This is the only ordering for which we have a closed form for a labelling of the simple modules for the quiver Hecke algebra [25] (in other words, a labelling of the component of the $\widehat{\mathfrak{s l}}_{e}$ crystal containing the empty $\ell$-partition). Notice that this combinatorial ordering and the algebraic structures it underlies are completely different from the classical dominance order and the (graded) cyclotomic $q$-Schur algebra [17,34,56]; see [5,59] for more details.

Given $h \in \mathbb{N}$, we let $\mathscr{P}_{n}^{\ell}(h)$ denote the subset of $\mathscr{P}_{n}^{\ell}$ consisting of those $\ell$-partitions which have at most $h$ columns in each component, that is

$$
\mathscr{P}_{n}^{\ell}(h)=\left\{\lambda=\left(\lambda^{(0)}, \lambda^{(1)}, \ldots, \lambda^{(\ell-1)}\right) \mid \lambda_{1}^{(m)} \leq h \text { for } 0 \leq m<\ell\right\} .
$$

Given $\lambda \in \mathscr{P}_{n}^{\ell}$, we define its residue sequence, $\operatorname{res}(\lambda)$ to be the sequence obtained by recording the residues of the boxes of $\lambda$ according to the ordering $\leq_{\kappa}$ on boxes.

\subsection{The quiver Hecke and Cherednik algebras}

Definition 1.7 Let $\epsilon \ll \frac{1}{n \ell}$. To $(r, c, m) \in \lambda$ we associate $\mathbf{I}_{(r, c, m)}^{\kappa}=\operatorname{ct}(r, c, m)-$ $m / \ell-(r+c) \epsilon$. Given $\lambda \in \mathscr{P}_{n}^{\ell}$, we let $\mathbf{I}_{\lambda}^{\kappa}$ denote the disjoint union over the $\mathbf{I}_{(r, c, m)}^{\kappa}$ for $(r, c, m) \in \lambda$. We define a $\kappa$-diagram of type $G(\ell, 1, n)$ to be a frame $\mathbb{R} \times[0,1]$ with distinguished solid points on the northern and southern boundaries given by $\mathbf{I}_{\mu}^{\kappa}$ and $\mathbf{I}_{\lambda}^{\kappa}$ for some $\lambda, \mu \in \mathscr{P}_{n}^{\ell}$ and a collection of solid strands each of which starts at a northern point and ends at a southern point. Each solid strand carries a residue, $i \in \mathbb{Z} / e \mathbb{Z}$, say (and we refer to this as a solid $i$-strand). We further require that each solid strand has a mapping diffeomorphically to $[0,1]$ via the projection to the $y$-axis. Each solid strand is allowed to carry any number of dots.

We draw

(i) a "ghost $i$-strand" 1 unit to the right of each solid $i$-strand and a a "ghost dot" 1 unit to the right of each solid dot;

(ii) vertical red lines with $x$-coordinate $\kappa_{m}-m / \ell \in \mathbb{Q}$ each of which carries the residue $\kappa_{m}$ in $\mathbb{Z} / e \mathbb{Z}$ for $1 \leq m \leq \ell$ which we call a red $\kappa_{m}$-strand.

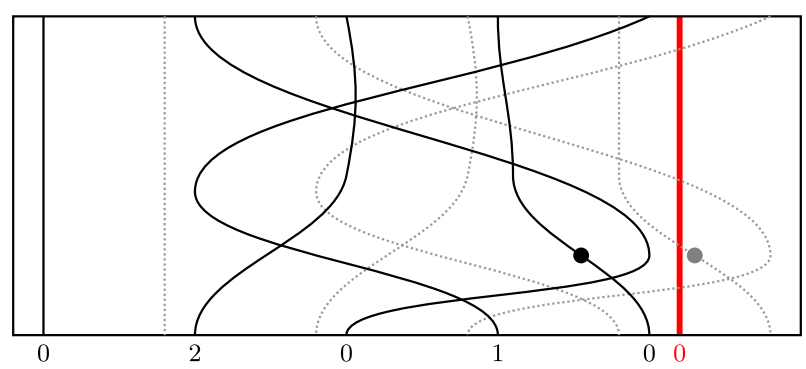

Fig. 1 A $\kappa$-diagram for $\ell=1$ and $\kappa \in \mathbb{Z}$ with northern and southern loading $\mathbf{I}_{\omega}^{\kappa}$ for $\omega=\left(1^{5}\right)$ 
Finally, we require that there are no triple points or tangencies involving any combination of strands, ghosts or red lines and no dots lie on crossings. We consider these diagrams equivalent if they are related by an isotopy that avoids these tangencies, double points and dots on crossings. See Fig. 1 for an example of a $\kappa$-diagram.

Remark 1.8 We emphasise that we have chosen $\epsilon \ll \frac{1}{n \ell}$ in order to guarantee that for any two boxes $(r, c, m),\left(r^{\prime}, c^{\prime}, m^{\prime}\right)$ for $1 \leq r, r^{\prime}, c, c^{\prime} \leq n$ and $0 \leq m<\ell$ we have that $\mathbf{I}_{(r, c, m)}^{\kappa} \neq \mathbf{I}_{\left(r^{\prime}, c^{\prime}, m^{\prime}\right)}^{\kappa}$.

Definition 1.9 We define the degree of a $\kappa$-diagram to be the integer obtained by summing over the degrees of all the local neighbourhoods of the diagram with each neighbourhood contributing either zero to the degree, or providing a non-zero degree contribution to the degree as follows:

$$
\operatorname{deg} \phi_{i}^{\dagger}=2 \quad \operatorname{deg} \chi_{i}=-2 \delta_{i, j} \quad \operatorname{deg} \underbrace{}_{i}=\delta_{j, i+1} \quad \operatorname{deg} \chi_{i}=\delta_{i, j}
$$

and their mirror images.

Definition 1.10 (Definition 4.1 [59]) We let $\mathbf{A}_{n}(\kappa)$ denote the $\mathbb{k}$-algebra spanned by all $\kappa$-diagrams modulo the following local relations (here a local relation means one that can be applied on a small region of the diagram). The product $d_{1} d_{2}$ of two diagrams $d_{1}, d_{2} \in A_{n}(\kappa)$ is given by putting $d_{1}$ on top of $d_{2}$. This product is defined to be 0 unless the southern border of $d_{1}$ matches the northern border of $d_{2}$, in which case we obtain a new diagram in the obvious fashion.

(A1) Any diagram may be deformed isotopically; that is, by a continuous deformation of the diagram which avoids tangencies, double points and dots on crossings.

(A2) Any solid dot can pass through a crossing of solid $i$ - and $j$-strands for $i \neq j$ or an arbitrary crossing involving a ghost strand. Namely:
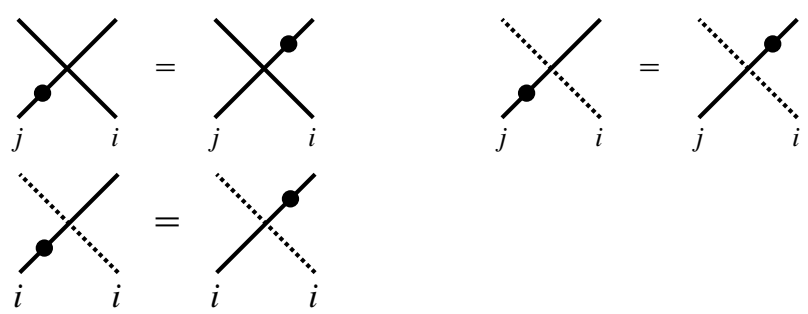

and their mirror images through reflection in the vertical axis hold.

(A3) We can pass a solid dot through a crossing of two like-labelled solid or ghost strands at the expense of an error term:
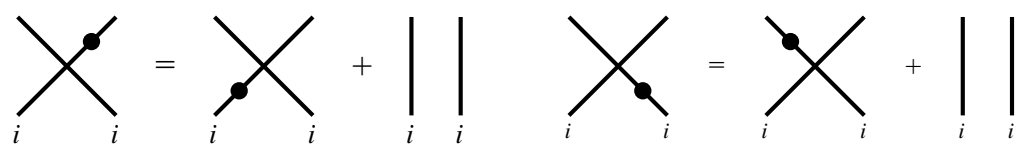
Ghost dots can pass through any crossing of strands (regardless of their residue) freely.

(A4) For double-crossings of solid strands with $i \neq j$, we have the following local relations:
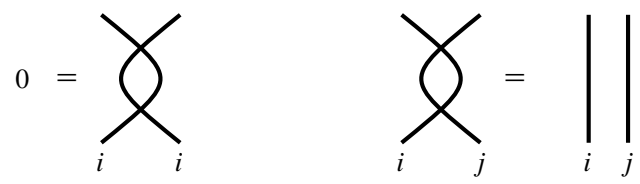

Performing relation (A4) implicitly involves undoing the corresponding doublecrossing of ghost strands at the same time (which we do not picture) and vice versa.

(A5) If $j \neq i-1$, then we can freely pass ghosts through solid strands. That is, we have the following local relations:

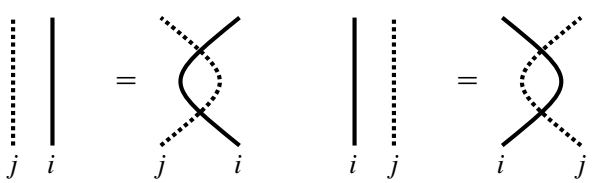

(A6) On the other hand, in the case where $j=i-1$, we have the following local relations:

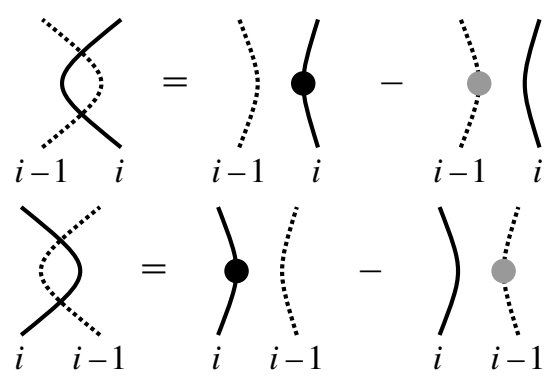

(A7) We can pull a solid $i$-strand through a $(i-1)$-ghost-crossing (or a ghost $(i-1)$ strand through a $i$-solid-crossing) at the expense of an error term.

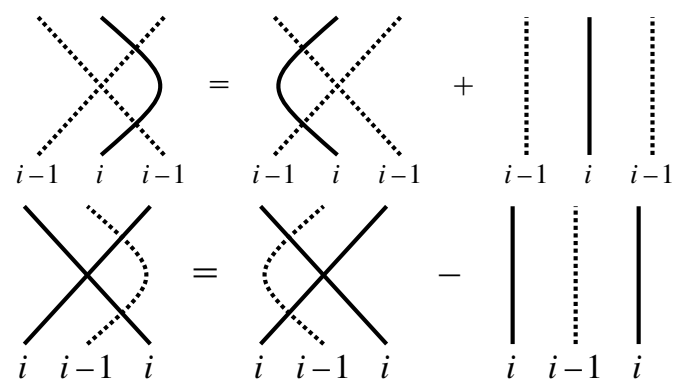


(A8) All other triples of solid and ghost strands satisfy the naive braid relation. Diagrammatically, we have that
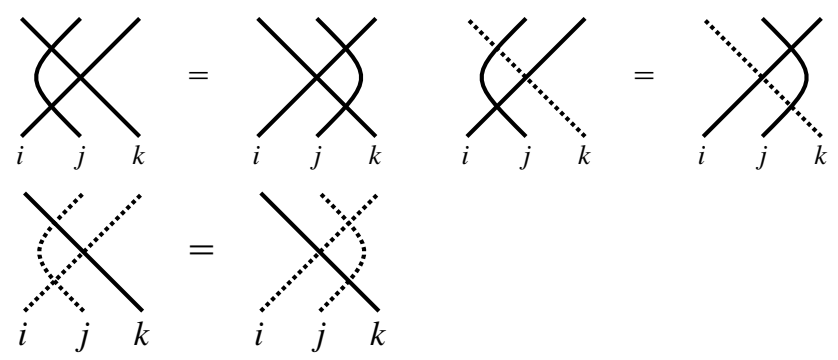

for any $i, j, k \in \mathbb{Z} / e \mathbb{Z}$ and their mirror images through reflection in the vertical axis hold. Performing the leftmost relation (A8) implicitly involves manipulating a braid of three ghost strands at the same time (which we do not picture) and vice versa. Furthermore,
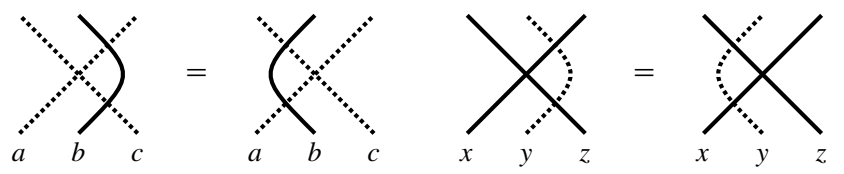

and $a, b, c, x, y, z \in \mathbb{Z} / e \mathbb{Z}$ such that $\delta_{a, b-1, c}=0, \delta_{x, y+1, z}=0$.

(A9) Ghost strands and ghost dots may pass through red strands freely. For $i \neq j$, the solid $i$-strands may pass through red $j$-strands freely. If the red and solid strands have the same label, a dot is added to the solid strand when straightening. Diagrammatically, we have that
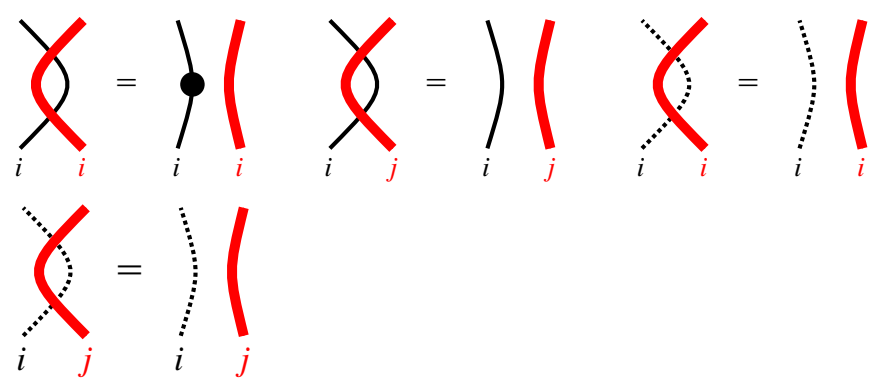

for $i \neq j$ and their mirror images through reflection through the vertical axis hold.

(A10) Solid crossings and dots can pass through red strands, with a correction term

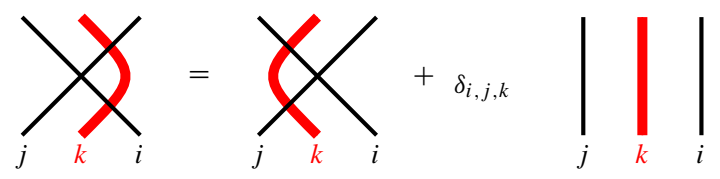


(A11) Any braid involving a red strand and not of the form in (A10) can be undone without cost. Diagrammatically, we have that
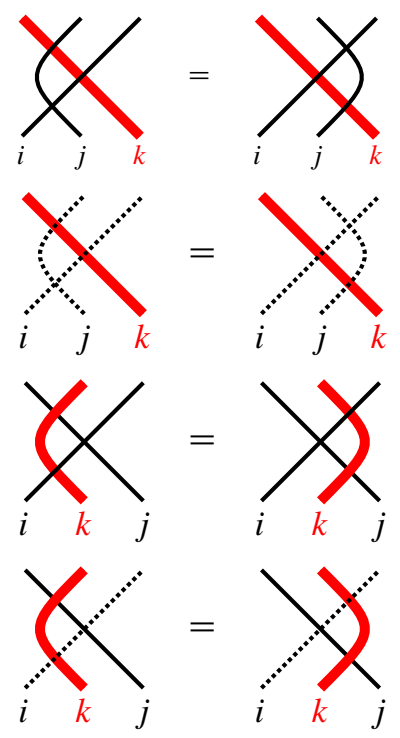
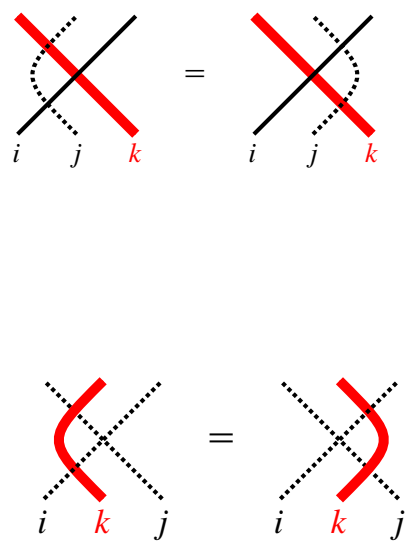

for any $i, j, k$ and their mirror images through reflection in the vertical axis hold.

(A12) Finally, any solid or ghost dot can be pulled through a red strand without cost. Diagrammatically, we have that
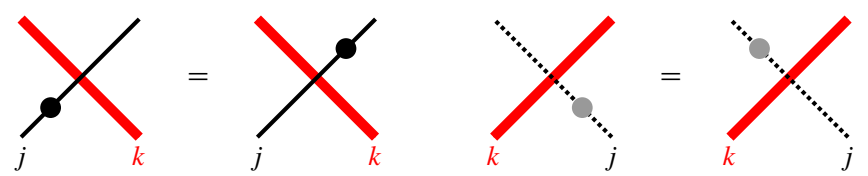

for any $j, k$ and their mirror images through reflection in the vertical axis hold. (We have not added the residues as they play no role here.)

Finally, we have the following non-local idempotent relation.

(A13) Any idempotent in which a solid strand is $n$ units to the right of the rightmost red-strand is referred to as unsteady and set to be equal to zero.

Given $\lambda \in \mathscr{P}_{n}^{\ell}$ and $\underline{i} \in(\mathbb{Z} / e \mathbb{Z})^{n}$, we have an associated weight-idempotent $1 \frac{i}{\lambda}$ given by the diagram with northern and southern points $\check{\mathbf{I}}$, no crossing strands, and northern/southern residue sequence of the diagram given by $\underline{i} \in(\mathbb{Z} / e \mathbb{Z})^{n}$. If the residue sequence is equal to that of the partition, $\operatorname{res}(\lambda)$, then we let $1_{\lambda}:=1_{\lambda}^{\operatorname{res}(\lambda)}$. We define the diagrammatic Cherednik algebra or weighted KLR algebra, $A_{n}(\kappa)$ to be the algebra

$$
A_{n}(\kappa):=\mathrm{E}^{+} \mathbf{A}_{n}(\kappa) \mathrm{E}^{+} \quad \text { where } \mathrm{E}^{+}=\sum_{\lambda \in \mathscr{P}_{n}^{\ell}} 1_{\lambda} .
$$


Definition 1.11 Let $\lambda, \mu \in \mathscr{P}_{n}^{\ell}$. A $\lambda$-tableau of weight $\mu$ is a bijective map $\mathrm{T}: \lambda \rightarrow \mathbf{I}_{\mu}^{\kappa}$. We let $\mathcal{T}(\lambda, \mu)$ denote the set of all tableaux of shape $\lambda$ and weight $\mu$.

We say that a tableau $\mathrm{T}$ is semistandard if it satisfies the following additional properties

(i) $\mathrm{T}(1,1, m)<\kappa_{m}$,

(ii) $\mathrm{T}(r, c, m)<\mathrm{T}(r-1, c, m)-1$,

(iii) $\mathrm{T}(r, c, m)<\mathrm{T}(r, c-1, m)+1$.

We denote the set of all semistandard tableaux of shape $\lambda$ and weight $\mu$ by $\operatorname{SStd}(\lambda, \mu)$. Given $\mathrm{T} \in \operatorname{SStd}(\lambda, \mu)$, we write $\operatorname{Shape}(\mathrm{T})=\lambda$. We let $\operatorname{SStd}_{n}^{+}(\lambda, \mu) \subseteq \operatorname{SStd}_{n}(\lambda, \mu)$ denote the subset of tableaux which respect residues. In other words, if $\mathrm{T}(r, c, m)=$ $\left(r^{\prime}, c^{\prime}, m^{\prime}\right)$ for $(r, c, m) \in \lambda$ and $\left(r^{\prime}, c^{\prime}, m^{\prime}\right) \in \mu$, then $\kappa_{m}+c-r=\kappa_{m^{\prime}}+c^{\prime}-r^{\prime}$ $(\bmod e)$.

Definition 1.12 Given $S$ a tableau of shape $\lambda$ and weight $\mu$, we let $C_{S}$ denote any diagram tracing out the bijection $S:[\lambda] \rightarrow \mathbf{I}_{\mu}^{\kappa}$ using the minimal number of crossings. Given S, T a pair of tableau of shape $\lambda$ (and possibly distinct weights) we set $C_{\mathrm{ST}}=$ $C_{\mathrm{S}} C_{\mathrm{T}}^{*}$ where $C_{\mathrm{T}}^{*}$ is the diagram obtained from $C_{\mathrm{T}}$ by flipping it through the horizontal axis.

Theorem 1.13 The R-algebra $\mathbf{A}_{n}(\kappa)$ is a quasi-hereditary graded cellular algebra with basis

$$
\left\{C_{\mathrm{ST}} \mid \mathrm{S} \in \operatorname{SStd}(\lambda, \mu), \mathrm{T} \in \operatorname{SStd}(\lambda, v), \lambda, \mu, v \in \mathscr{P}_{n}^{\ell}\right\}
$$

and the subalgebra $A_{n}(\kappa)$ is a quasi-hereditary graded cellular algebra with basis

$$
\left\{C_{\mathrm{ST}} \mid \mathrm{S} \in \mathrm{SStd}^{+}(\lambda, \mu), \mathrm{T} \in \mathrm{SStd}^{+}(\lambda, v), \lambda, \mu, v \in \mathscr{P}_{n}^{\ell}\right\} .
$$

For both algebras, the involution is given by $*$ and the ordering on $\mathscr{P}_{n}^{\ell}(h)$ is that of Definition 1.2. We denote the corresponding left $\mathbf{A}_{n}(\kappa)$ - and $A_{n}(\kappa)$-cell-modules are

$$
\boldsymbol{\Delta}_{\mathbf{A}_{n}(\kappa)}(\lambda), \quad \Delta_{A_{n}(\kappa)}(\lambda)
$$

respectively. These modules have simple heads, denoted by

$$
\mathbf{L}_{\mathbf{A}_{n}(\kappa)}(\lambda), \quad L_{A_{n}(\kappa)}(\lambda)
$$

respectively. When the context is clear, we drop the subscripts on these modules.

We let $A_{n}^{\triangleright \lambda}(\kappa)$ denote the 2 -sided cell-ideal $\sum_{\lambda \triangleright \lambda} A_{n}(\kappa) 1_{\lambda} A_{n}(\kappa)$.

The standard tableaux $\operatorname{Std}(\lambda)$ form the predictable subset of semistandard tableaux of weight $\omega=\left(1^{n}\right)$ as follows. Let $\lambda \in \mathscr{P}_{n}^{\ell}$. If $\mathrm{s} \in \operatorname{Std}(\lambda)$ is such that $\mathrm{s}\left(r_{k}, c_{k}, m_{k}\right)=k$ for $1 \leq k \leq n$, then we let $S \in \mathcal{T}(\lambda, \omega)$ denote the tableau $S: \lambda \rightarrow \omega$ determined by $\mathrm{S}\left(r_{k}, c_{k}, m_{k}\right)=\mathbf{I}_{(k, 1, \ell)}^{\kappa}$. We have a bijective $\operatorname{map} \varphi: \operatorname{Std}(\lambda) \rightarrow \mathcal{T}(\lambda, \omega)$. given by $\varphi(\mathrm{s})=\mathrm{S}$. 
Definition 1.14 We define the Schur idempotent, $\mathrm{E}_{\omega}$, and quiver Hecke algebra, $H_{n}(\kappa)$, as follows

$$
\mathrm{E}_{\omega}=\sum_{\underline{i} \in(\mathbb{Z} / e \mathbb{Z})^{n}} 1 \frac{i}{\omega} \quad \text { and } \quad H_{n}(\kappa):=\mathrm{E}_{\omega} \mathbf{A}_{n}(\kappa) \mathrm{E}_{\omega} .
$$

Theorem 1.15 ([5]) The algebra $H_{n}(\kappa)$ admits a graded cellular structure with respect to the poset $\left(\mathscr{P}_{n}^{\ell}, \triangleright\right)$, the basis

$$
\left\{c_{\mathrm{st}}:=C_{\varphi(\mathrm{s}), \varphi(\mathrm{t})} \mid \lambda \in \mathscr{P}_{n}^{\ell}, \mathrm{s}, \mathrm{t} \in \operatorname{Std}(\lambda)\right\}
$$

and the involution $*$. We denote the left cell-module by $S_{n}(\lambda):=\mathrm{E}_{\omega} \Delta(\lambda)$ with basis $\left\{c_{\mathrm{s}} \mid \mathrm{s} \in \operatorname{Std}(\lambda)\right\}$ and let $D_{n}(\lambda)$ denote the simple head of $S_{n}(\lambda)$.

When proving results on homomorphisms, the algebra $A_{n}(\kappa)$ is smaller than $\mathbf{A}_{n}(\kappa)$ and much easier for computation. We shall then (trivially) inflate these results to $\mathbf{A}_{n}(\kappa)$ and apply the Schur functor to obtain the corresponding result for $H_{n}(\kappa)$.

Remark 1.16 We let $t$ be an indeterminate over $\mathbb{Z}_{\geq 0}$. If $M=\oplus_{k \in \mathbb{Z}} M_{k}$ is a graded $\mathbb{k}$-module, we write $\operatorname{dim}_{t}(M)=\sum_{k \in \mathbb{Z}}\left(\operatorname{dim}_{\mathbb{k}}\left(M_{k}\right)\right) t^{k}$.

Finally, we briefly recall from [6, Theorem 4.9] that for the above three algebras, there are graded Morita equivalences relating the subcategories of $\mathbf{A}_{n}(\kappa)$-mod, $A_{n}(\kappa)$-mod, and $H_{n}(\kappa)$-mod whose simple constituents are labelled by $\mathscr{P}_{n}^{\ell}(h)$. In particular

$$
\operatorname{Hom}_{A_{n}(\kappa)}(\Delta(\mu), \Delta(\lambda)) \cong \operatorname{Hom}_{\mathbf{A}_{n}(\kappa)}(\boldsymbol{\Delta}(\mu), \boldsymbol{\Delta}(\lambda)) \cong \operatorname{Hom}_{H_{n}(\kappa)}\left(S_{n}(\mu), S_{n}(\lambda)\right)
$$

for $\lambda, \mu \in \mathscr{P}_{n}^{\ell}(h)$. This allows us to focus on the algebras $A_{n}(\kappa)$ where we have the benefit of a highest weight theory which is intimately related to the underlying alcove geometry and $H_{n}(\kappa)$ where we have more easily constructible restriction functors, which we will recall momentarily. Both isomorphisms are simply given by idempotent truncation from $\mathbf{A}_{n}(\kappa)$. The truncation from $\mathbf{A}_{n}(\kappa)$ to $H_{n}(\kappa)$ kills every weight-idempotent $1 \frac{i}{\lambda}$ for $\underline{i} \in(\mathbb{Z} / e \mathbb{Z})^{n}$ and $\omega \neq \lambda \in \mathscr{P}_{n}^{\ell}$.

\subsection{Induction and restriction}

For $r \in \mathbb{Z} / e \mathbb{Z}$, we have an embedding $\iota_{n-1}^{r}: H_{n-1}(\kappa) \hookrightarrow H_{n}(\kappa)$. Given $D \in H_{n-1}(\kappa)$ the diagram $\iota_{n-1}^{r}$ is obtained by adding a single solid $r$-strand (connecting the northern and southern points $\left.\mathbf{I}_{(n, 1, \ell)}^{\kappa}\right)$ and its ghost to the left of all strands in $D$.

Example 1.17 For example, the diagram in Fig. 1 is of the form $\iota_{4}^{0}(D)$ for $D \in H_{4}(0)$.

We let $\iota_{n-1}=\sum_{r \in \mathbb{Z} / e \mathbb{Z}} \iota_{n-1}^{r}$. We have that $H_{n}(\kappa)$ is free as a $H_{n-1}(\kappa)$-module under the identification $H_{n-1}(\kappa) \cong \iota_{n-1}\left(H_{n-1}(\kappa)\right)$. We let res ${ }_{n-1}^{n}: H_{n}(\kappa)$-mod $\rightarrow$ 
$H_{n-1}(\kappa)$-mod and $\operatorname{ind}_{n-1}^{n}: H_{n-1}(\kappa)$-mod $\rightarrow H_{n}(\kappa)$-mod denote the obvious restriction and induction functors. We post-compose these functors with the projection onto a block in the standard fashion. This amounts to multiplying by an idempotent

$$
\mathrm{E}_{\omega}^{r}=\sum_{\underline{i}=\left(i_{1}, \ldots, i_{n-1}, r\right)} 1 \frac{i}{\omega}
$$

for $r \in \mathbb{Z} / e \mathbb{Z}$. We hence decompose these restriction/induction functors into

$$
r-\operatorname{res}_{n-1}^{n}=\mathrm{E}_{\omega}^{r} \circ \operatorname{res}_{n-1}^{n} \quad r-\text { ind }_{n-1}^{n}=\mathrm{E}_{\omega}^{r} \circ \operatorname{ind}_{n-1}^{n}
$$

Finally we recall a simple case of [5, Theorem 12.1]. If $\lambda$ has precisely one removable $r$-box, $\square \in \operatorname{Rem}_{r}(\lambda)$, then we set $\mathrm{E}_{\omega}^{r}(\lambda)=\lambda-\square$ and we have that

$$
S_{n-1}(\lambda-\square) \cong r \text {-res }_{n-1}^{n}\left(S_{n}(\lambda)\right)
$$

This isomorphism is given by "ignoring the leftmost strand" as follows

$$
C_{\mathrm{S}} \mapsto \iota_{n-1}^{r}\left(C_{\mathrm{S}}\right) \times C_{\mathrm{T}_{\square}}
$$

for $S \in \operatorname{SStd}\left(\lambda-\square,\left(1^{n-1}\right)\right)$. For more details we refer to [5, Section 12].

Remark 1.18 We note that our embeddings are "adding strands to the left" whereas many readers are used to "adding strands to the right". This is because our algebras are build from the natural ordering Definition1.2. By building diagrams from the opposite order on contents, one can obtain embeddings which "add strands to the right".

Remark 1.19 The subalgebra $H_{n}(\kappa)=\mathrm{E}_{\omega} \mathbf{A}_{n}(\kappa) E_{\omega}$ is usually defined via generators $e(\underline{i}), \psi_{r}$, and $y_{r}$ subject to certain relations (see [5, Definition 3.1] for more details). We will make use of this classical notation by identifying the generator $\sigma(e(i)$ with $1 \frac{i}{w}$ while $\sigma\left(y_{r} e(i)\right)$ gets identified with

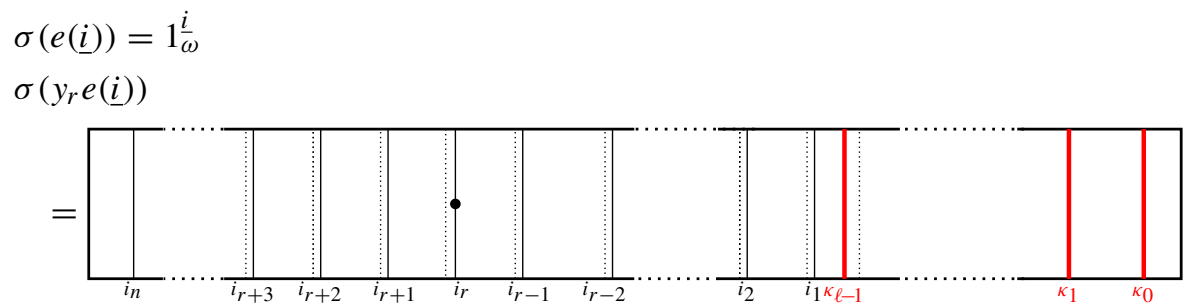

$\sigma\left(\psi_{r} e(\underline{i})\right)$

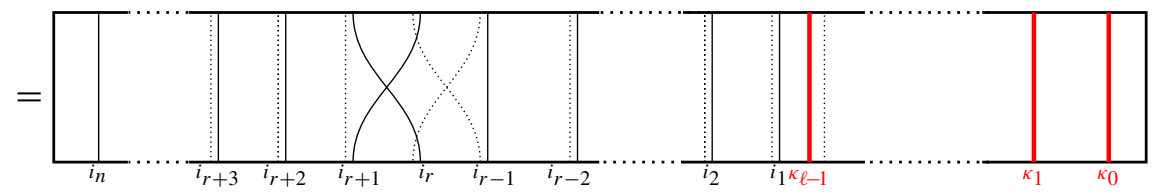




\section{Alcove geometries and path-bases of diagrammatic algebras}

In Subsection 2.1, we recall the alcove geometry controlling the subcategories of representations for quiver Hecke and Cherednik algebras of interest in this paper. In Subsection 2.2 we cast the semistandard tableaux for diagrammatic Cherednik algebras in this geometry; this path-combinatorial framework will be essential for our proofs. In Subsection 2.3 we cast the standard tableaux combinatorics of the quiver Hecke algebra in this geometry - this allows us to define the $e$-restricted tableaux which we will prove provide bases of simple $H_{n}(\kappa)$-modules in Sect. 4.

\subsection{The alcove geometry}

Fix integers $h, \ell \in \mathbb{Z}_{>0}$ and $e \geq h \ell$. For each $1 \leq i \leq h$ and $0 \leq m<\ell$ we let $\varepsilon_{h m+i}$ denote a formal symbol, and define an $\ell h$-dimensional real vector space,

$$
\mathbb{E}_{h, \ell}=\bigoplus_{\substack{1 \leq i \leq h \\ 0 \leq m<\ell}} \mathbb{R} \varepsilon_{h m+i}
$$

We have an inner product $\langle$,$\rangle given by extending linearly the relations$

$$
\left\langle\varepsilon_{h m+i}, \varepsilon_{h t+j}\right\rangle=\delta_{i, j} \delta_{t, m}
$$

for all $1 \leq i, j \leq h$ and $0 \leq m, t<\ell$, where $\delta_{i, j}$ is the Kronecker delta. We let $\Phi$ and $\Phi_{0}$ denote the root systems of type $A_{\ell h-1}$ and $A_{h-1} \times A_{h-1} \times \ldots A_{h-1}$ respectively which consist of the roots

$$
\begin{aligned}
& \left\{\varepsilon_{h m+i}-\varepsilon_{h t+j} \mid 1 \leq i, j \leq h \text { and } 0 \leq m, t<\ell \text { with }(i, m) \neq(j, t)\right\} \text { and } \\
& \left\{\varepsilon_{h m+i}-\varepsilon_{h m+j} \mid 1 \leq i, j \leq h \text { with } i \neq j \text { and } 0 \leq m<\ell\right\}
\end{aligned}
$$

respectively. We identify $\lambda \in \mathscr{P}_{n}^{\ell}(h)$ with a point in $\mathbb{E}_{h, \ell}$ via the transpose map

$$
\left(\lambda^{(0)}, \ldots, \lambda^{(\ell-1)}\right) \mapsto \sum_{\substack{1 \leq i \leq \ell \\ 1 \leq j \leq h}}\left(\lambda^{(m)}\right)_{i}^{T} \varepsilon_{h m+i},
$$

(where the $T$ denotes the transpose partition). Given $r \in \mathbb{Z}$ and $\alpha \in \Phi$ we let $s_{\alpha, r e}$ denote the reflection which acts on $\mathbb{E}_{h, \ell}$ by

$$
s_{\alpha, r e} x=x-(\langle x, \alpha\rangle-r e) \alpha .
$$

and we let $W_{e}$ and $W_{0}$ denote the Coxeter groups generated by the reflections

$$
\mathcal{S}=\left\{s_{\alpha, 0} \mid \alpha \in \Phi\right\} \cup\left\{s_{\varepsilon_{h}-\varepsilon_{1},-e}\right\} \quad \mathcal{S}_{0}=\left\{s_{\alpha, 0} \mid \alpha \in \Phi_{0}\right\}
$$


respectively. We let $\ell: W_{e} \rightarrow \mathbb{N}$ denote the length function and we let $\leq$ denote the Bruhat ordering for the Coxeter group $W_{e}$. For $e \in \mathbb{Z}_{>0}$ we assume that $\kappa \in \mathbb{Z}^{\ell}$ is $h$-admissible. We shall consider a shifted action of the Weyl group $W^{e}$ on $\mathbb{E}_{h, \ell}$ by the element

$\rho:=\left(\rho_{1}, \rho_{2}, \ldots, \rho_{\ell}\right) \in \mathbb{Z}_{\geq 0}^{h \ell}, \quad \rho_{i}:=\left(e-\kappa_{i}, e-\kappa_{i}-1, \ldots, e-\kappa_{i}-h+1\right) \in \mathbb{Z}_{\geq 0}^{h}$,

that is, given an an element $w \in W^{e}$, we set $w \cdot \rho x=w(x+\rho)-\rho$. We let $\mathbb{E}(\alpha, r e)$ denote the affine hyperplane consisting of the points

$$
\mathbb{E}(\alpha, r e)=\left\{x \in \mathbb{E}_{h, \ell} \mid s_{\alpha, r e} \cdot x=x\right\} .
$$

The connected components of $\mathbb{E}_{h, \ell} \backslash\left(\cup_{\alpha \in \Phi, r \in \mathbb{Z}} \mathbb{E}(\alpha, r e)\right)$ are called alcoves. Note that our assumption that $\kappa \in \mathbb{Z}_{\geq 0}^{\ell}$ is $h$-admissible implies that the origin lies in an alcove. Given a hyperplane $\mathbb{E}(\alpha, r e)$ we remove the hyperplane from $\mathbb{E}_{h, \ell}$ to obtain two distinct subsets $\mathbb{E}^{>}(\alpha, r e)$ and $\mathbb{E}^{<}(\alpha, r e)$ where the origin $\odot \in \mathbb{E}^{<}(\alpha, r e)$. The dominant Weyl chamber, denoted $\mathbb{E}_{h, \ell}^{\odot}$, is set to be

$$
\mathbb{E}_{h, \ell}^{\ominus}=\bigcap_{\alpha \in \Phi_{0}} \mathbb{E}^{<}(\alpha, 0) .
$$

Definition 2.1 Let $\lambda \in \mathbb{E}_{h, \ell}$. There are only finitely many hyperplanes lying between the point $\lambda \in \mathbb{E}_{h, \ell}$ and the point $v \in \mathbb{E}_{h, \ell}$. For a root $\varepsilon_{i}-\varepsilon_{j} \in \Phi$, we let $\ell_{\varepsilon_{i}-\varepsilon_{j}}(\lambda, \nu)$ denote the total number of these hyperplanes which are perpendicular to $\varepsilon_{i}-\varepsilon_{j} \in \Phi$ (excluding any hyperplanes upon which $\lambda$ or $v$ lies). We let $\ell(\lambda, v)=\sum_{1 \leq i<j \leq h \ell} \ell_{\varepsilon_{i}-\varepsilon_{j}}(\lambda, \nu)$. We let $\ell(\lambda):=\ell(\lambda, \odot)$ for $\odot$ the origin and refer to $\ell(\lambda)$ simply as the length of the point $\lambda \in \mathbb{E}_{h, \ell}$.

Definition 2.2 Given $n \in \mathbb{N}$, we define the fundamental alcove to be

$$
\mathcal{F}_{n}^{\ell}(h)=\left\{\lambda \in \mathscr{P}_{n}^{\ell} \mid \ell(\lambda)=0\right\}
$$

We have that $W_{e}$ acts on the set of all alcoves. We can uniquely write any alcove, $A$, in the form $A=w \mathcal{F}_{n}^{\ell}(h)$ for some coset representative $w$ in $W_{0} \backslash W_{e}$. This provides an important labelling for alcoves. For $\lambda \in w \mathcal{F}_{n}^{\ell}(h)$, we have that $\ell(\lambda)=\ell(w)$.

Proposition 2.3 Fix integers $h, \ell \in \mathbb{Z}_{>0}$ and $e \geq h \ell$ and suppose $\kappa \in \mathbb{Z}^{\ell}$ is $h$ admissible. A necessary condition for $L(\lambda)$ and $L(\mu)$ to belong to the same block is that they belong to the same $W_{e}$-linkage class, that is $\lambda \in W_{e} \cdot \mu$. Given $\lambda \in w \mathcal{F}_{n}^{\ell}(h)$ and $\mu \in w^{\prime} \mathcal{F}_{n}^{\ell}(h)$, we have that $\mu \unlhd_{\kappa} \lambda$ if and only if $w \leq w^{\prime}$.

Proof A necessary condition for $L(\lambda)$ and $L(\mu)$ to belong to the same block is that $\operatorname{res}(\lambda)=\operatorname{res}(\mu)$; to see this simply note that the basis elements in equation (1.2) respect residues. Reflection through a hyperplane $\mathbb{E}\left(\varepsilon_{h m+i}-\varepsilon_{h t+j}, r e\right)$ corresponds to a (residue preserving) swap of boxes between the $i$ th column of the $m$ th component and the $j$ th column of the $t$ th component. The result follows. 
Definition 2.4 Given a map $s:\{1, \ldots, n\} \rightarrow\{1, \ldots, \ell h\}$ we define points $S(k) \in \mathbb{E}_{h, \ell}$ by $S(k)=\sum_{1 \leq i \leq k} \varepsilon_{S(i)}$ for $1 \leq k \leq n$. We define the associated path of length $n$ in $\mathbb{E}_{h, \ell}$ by

$$
\mathrm{S}=(\mathrm{S}(0), \mathrm{S}(1), \mathrm{S}(2), \ldots, \mathrm{S}(n))
$$

where we fix all paths to begin at the origin, so that $\mathrm{S}(0)=\odot \in \mathbb{E}_{h, \ell}$. We let $\mathrm{S}_{\leq k}$ denote the subpath of $S$ of length $k$ corresponding to the restriction of the map $s$ to the domain $\{1, \ldots, k\} \subseteq\{1, \ldots, n\}$. We let Shape(S) denote the point in $\mathbb{E}_{h, \ell}$ at which $\mathrm{S}$ terminates.

Remark 2.5 Let $S$ be a path which passes through a hyperplane $\mathbb{E}_{\alpha, \text { re }}$ at point $S(k)$ (note that $k$ is not necessarily unique). Let $\mathrm{T}$ be the path obtained from $S$ by applying the reflection $s_{\alpha, r e}$ to all the steps in $\mathrm{S}$ after the point $\mathrm{S}(k)$. In other words, $\mathrm{T}(i)=\mathrm{S}(i)$ for all $1 \leq i \leq k$ and $\mathrm{T}(i)=s_{\alpha, r e} \cdot \mathrm{S}(i)$ for $k \leq i \leq n$. We refer to the path $\mathrm{T}$ as the reflection of $\mathrm{S}$ in $\mathbb{E}_{\alpha, r e}$ at point $\mathrm{S}(k)$ and denote this by $s_{\alpha, r e}^{k} \cdot \mathrm{S}$. We write $\mathrm{S} \sim \mathrm{T}$ if the path $T$ can be obtained from $S$ by a series of such reflections.

Definition 2.6 Let T denote a fixed path from $\odot$ to $v \in \mathscr{P}_{n}^{\ell}(h)$. We let $\operatorname{Path}_{n}(\lambda, \mathrm{T})$ denote the set of paths from the origin to $\lambda$ obtainable by applying repeated reflections to $\mathrm{T}$, in other words

$$
\operatorname{Path}_{n}(\lambda, \mathrm{T})=\{\mathrm{S} \mid \mathrm{S}(n)=\lambda, \mathrm{S} \sim \mathrm{T}\}
$$

We let $\operatorname{Path}_{n}^{+}(\lambda, \mathrm{T}) \subseteq \operatorname{Path}_{n}(\lambda, \mathrm{T})$ denote the set of paths which at no point leave the dominant Weyl chamber, in other words

$$
\operatorname{Path}_{n}^{+}(\lambda, \mathrm{T})=\left\{\mathrm{S} \in \operatorname{Path}_{n}(\lambda, \mathrm{T}) \mid \mathrm{S}(k) \in \mathbb{E}_{h, \ell}^{\odot} \text { for all } 1 \leq k \leq n\right\}
$$

Definition 2.7 Given a path $\mathrm{S}=(\mathrm{S}(0), \mathrm{S}(1), \mathrm{S}(2), \ldots, \mathrm{S}(n))$, we define $d(\mathrm{~S}(k), \mathrm{S}(k-$ 1)) as follows. For $\alpha \in \Phi$ we set $d_{\alpha}(\mathrm{S}(k), \mathrm{S}(k-1))$ to be

- +1 if $\mathrm{S}(k-1) \in \mathbb{E}(\alpha, r e)$ and $\mathrm{S}(k) \in \mathbb{E}^{<}(\alpha, r e)$;

- -1 if $\mathrm{S}(k-1) \in \mathbb{E}^{>}(\alpha, r e)$ and $\mathrm{S}(k) \in \mathbb{E}(\alpha, r e)$;

- 0 otherwise.

We set $\operatorname{deg}(S(0))=0$ and define

$d(\mathrm{~S}(k-1), \mathrm{S}(k))=\sum_{\alpha \in \Phi} d_{\alpha}(\mathrm{S}(k-1), \mathrm{S}(k)) \quad$ and $\quad \operatorname{deg}(\mathrm{S})=\sum_{1 \leq k \leq n} d(\mathrm{~S}(k), \mathrm{S}(k-1))$

\subsection{Semistandard tableaux as paths}

Let $e>h \ell$. We now provide path-theoretic bases for the diagrammatic Cherednik algebra. Let $\mu \in \mathscr{P}_{n}^{\ell}(h)$. We define the component word of $\mu$ to be the series of $\ell$-partitions 


$$
\varnothing=\mu^{(0)} \stackrel{+X_{1}}{\longrightarrow} \mu^{(1)} \stackrel{+X_{2}}{\longrightarrow} \mu^{(2)} \stackrel{+X_{3}}{\longrightarrow} \cdots \stackrel{+X_{n-1}}{\longrightarrow} \mu^{(n-1)} \stackrel{+X_{n}}{\longrightarrow} \mu^{(n)}=\mu
$$

where $X_{k}=\left(r_{k}, c_{k}, m_{k}\right)$ is the minimal node, with respect to $\leq_{\kappa}$, of the partition $\mu^{(k)} \in \mathscr{P}_{k}^{\ell}(h)$. Using the component word of $\mu$, we define a distinguished path $\mathrm{T}^{\mu}$ from the origin to $\mu$ as follows

$$
\mathrm{T}^{\mu}=\left(+\varepsilon_{X_{1}},+\varepsilon_{X_{2}}, \ldots,+\varepsilon_{X_{n}}\right) .
$$

For $\lambda \in \mathscr{P}_{n}^{\ell}(h)$, we let

$$
\mathrm{S}=\left(+\varepsilon_{Y_{1}},+\varepsilon_{Y_{2}}, \ldots,+\varepsilon_{Y_{n}}\right) \in \operatorname{Path}\left(\lambda, \mathrm{T}^{\mu}\right) .
$$

From $S$, we obtain a tableau $\overline{\mathrm{S}} \in \mathcal{T}(\lambda, \mu)$ by setting $\overline{\mathrm{S}}\left(X_{k}\right)=\mathbf{I}_{Y_{k}}$. We freely identify paths and tableaux in this manner (and so we drop the overline). Under this identification, we obtain a bijection $\operatorname{SStd}^{+}(\lambda, \mu) \leftrightarrow \operatorname{Path}^{+}\left(\lambda, \mathrm{T}^{\mu}\right)$ and hence we can rewrite the basis of Theorem 1.13 in terms of paths (see [6, Theorem 5.21]) as follows. For $\lambda \in \mathscr{P}_{n}^{\ell}(h)$ we have that

$$
\Delta(\lambda)=\left\{C_{\mathrm{S}} \mid \mathrm{S} \in \operatorname{Path}^{+}\left(\lambda, \mathrm{T}^{\mu}\right), \mu \in \mathscr{P}_{n}^{\ell}(h)\right\} .
$$

Definition 2.8 Let $\lambda, \mu \in \mathscr{P}_{n}^{\ell}(h)$ and suppose that $\lambda \triangleright \mu$. Then we let $\mathrm{T}_{\lambda}^{\mu} \in$ $\operatorname{Path}\left(\lambda, \mathrm{T}^{\mu}\right)$ denote the unique path satisfying

$$
\operatorname{deg}\left(\mathrm{T}_{\lambda}^{\mu}\right)=\ell(\mu)-\ell(\lambda) .
$$

The above definition is well-defined by [6, Proposition 7.4] and these paths will be very useful later on. Examples of this path/tableau for three distinct pairs $(\lambda, \mu)$ are given in Fig. 2.

Remark 2.9 If $e=h \ell$, then all the results of this paper go through unchanged modulo minor edits to the proofs. Annoyingly, the definition of the degree and reflections of paths require some tinkering (akin to the case $e=\infty$ case covered in detail in [6, Section 6.4]). In what follows, we only discuss the case $e>h \ell$ explicitly. For Cherednik algebras of symmetric groups, we provide an explicit and independent proof of our main result in quantum characteristic $e=h$ in Subsection 6.1.

\subsection{Standard tableaux as paths}

Given $\lambda \in \mathscr{P}_{n}^{\ell}(h)$, a tableau $\mathrm{t} \in \operatorname{Std}(\lambda)$ is easily identified with the series of partitions $\mathrm{t}(k)$ for $0 \leq k \leq n$, which in turn determine a path in $\mathbb{E}_{h, \ell}^{+}$via the map in Eq. (2.1). This provides path-theoretic bases of Specht modules $S_{n}(\lambda)$ for $\lambda \in \mathscr{P}_{n}^{\ell}(h)$. We now restrict our attention to $\lambda \in \mathcal{F}_{n}^{\ell}(h) \subseteq \mathscr{P}_{n}^{\ell}(h)$ and define the subset of $e$-restricted standard $\lambda$-tableaux which will index the basis of the simple module $D_{n}(\lambda)$ for $\lambda \in \mathcal{F}_{n}^{\ell}(h)$.

Definition 2.10 Given $\lambda \in \mathcal{F}_{n}^{\ell}(h)$, we say that $\mathrm{s} \in \operatorname{Std}(\lambda)$ is $e$-restricted if $\mathrm{s}(k) \in$ $\mathcal{F}_{k}^{\ell}(h)$ for all $1 \leq k \leq n$. We let $\operatorname{Std}_{e}(\lambda)$ denote the set of all $e$-restricted tableaux of shape $\lambda$. 
Fig. 2 The black points label the 3-partitions of a block of $H_{8}(0,1,2)$ with $e=4$. The origin is labelled as $\odot$. There are three separate paths drawn on the diagram belonging to $\operatorname{Path}^{+}\left(\left(1^{4}|\varnothing| 1^{4}\right), \mathrm{T}^{\left(1^{8}|\varnothing| \varnothing\right)}\right)$, Path $^{+}\left(\left(1^{6}|1| 1\right), \mathrm{T}^{\left(\varnothing\left|1^{8}\right| \varnothing\right)}\right)$, and Path ${ }^{+}\left(\left(1^{2}|1| 1^{5}\right)\right.$, $\left.\mathrm{T}^{\left(\varnothing|\varnothing| 1^{8}\right)}\right)$. These are coloured red, blue, and violet respectively. We have labelled some of the points in the diagram for reference (colour figure online)

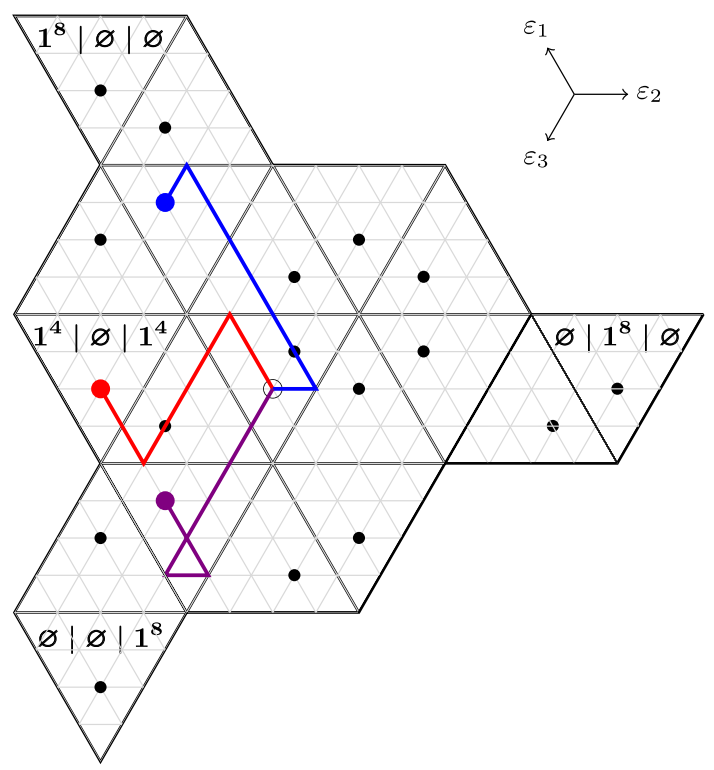

Given $\lambda \in \mathcal{F}_{n}^{\ell}(h)$, we say that a node $\square \in \operatorname{Rem}(\lambda)$ is good if $\lambda-\square \in \mathcal{F}_{n-1}^{\ell}(h)$ (we remark that this is easily seen to coincide with the classical definition of a good node). We let $\mathcal{F}_{h}(\lambda)$ denote the set of all good removable nodes of $\lambda$. The following result is obvious, but will be essential for the proof of our main theorem.

Proposition 2.11 Given $\lambda \in \mathcal{F}_{n}^{\ell}(h)$, we have that $\left\langle c_{\mathrm{s}}, c_{\mathrm{t}}\right\rangle=\delta_{\mathrm{s}, \mathrm{t}}$ for $\mathrm{s}, \mathrm{t} \in \operatorname{Std}_{e}(\lambda)$. Furthermore,

$$
\mathbb{k}\left\{c_{\mathrm{s}} \mid \mathrm{s} \in \operatorname{Std}_{e}(\lambda)\right\} \subseteq D_{n}(\lambda) \quad \text { and } \quad \operatorname{Std}_{e}(\lambda) \leftrightarrow \bigsqcup_{\square \in \mathcal{F}_{h}(\lambda)} \operatorname{Std}_{e}(\lambda-\square)
$$

Proof For $\lambda \in \mathcal{F}_{n}^{\ell}(h)$, we have that $s \in \operatorname{Std}_{e}(\lambda)$ if and only if $s \downarrow_{n-1} \in \operatorname{Std}_{e}(\nu)$ for some $v \in \mathcal{F}_{n-1}^{\ell}(h)$; the bijection follows. To see that $\left\{c_{\mathrm{s}} \mid \mathrm{s} \in \operatorname{Std}_{e}(\lambda)\right\} \subseteq D_{n}(\lambda)$ and that $\left\langle c_{\mathrm{s}}, c_{\mathrm{t}}\right\rangle=\delta_{\mathrm{s}, \mathrm{t}}$ for $\mathrm{s}, \mathrm{t} \in \operatorname{Std}_{e}(\lambda)$, it is enough to show that

$$
1_{\omega}^{\mathrm{res}(\mathrm{s})} S_{n}(v) \neq 0 \text { implies } v \triangleright \lambda \text { or } v=\lambda \text { and } 1_{\omega}^{\mathrm{res}(\mathrm{s})} S_{n}(\lambda)=c_{\mathrm{s}}
$$

for $\mathrm{s} \in \operatorname{Std}_{e}(\lambda)$. To see this, assume that $c_{\mathrm{s}}$ for $\mathrm{s} \in \operatorname{Std}_{e}(\lambda)$ belongs to some simple composition factor $L(v)$ of $S_{n}(\lambda)$ for $v \neq \lambda$; in which case $\lambda \triangleright v$ and

$$
1_{\omega}^{\mathrm{res}(\mathrm{s})} L(v) \subseteq 1_{\omega}^{\mathrm{res}(\mathrm{s})} S_{n}(v) \neq 0
$$

which gives us our required contradiction. Now we turn to the proof of Eq. (2.3). If $v \leqslant \lambda$, then $v \in \mathscr{P}_{n}^{\ell}(h)$. Given $\mathrm{t} \in \operatorname{Std}(v)$ with $\mathrm{t}\left(r_{k}, c_{k}, m_{k}\right)=k$, we identify $\mathrm{t}$ with the path

$$
\left(+\varepsilon_{h m_{1}+c_{1}},+\varepsilon_{h m_{2}+c_{2}},+\ldots,+\varepsilon_{h m_{n}+c_{n}}\right) .
$$


Given $\mathrm{t} \in \operatorname{Std}(v)$, we have that

$$
\operatorname{Path}^{+}(\lambda, t)=\{u \in \operatorname{Std}(\lambda) \mid \operatorname{res}(u)=\operatorname{res}(t)\}
$$

Given any $\mathrm{s} \in \operatorname{Std}_{e}(\lambda)$, we have that $\mathrm{s}(k) \in \mathcal{F}_{k}^{\ell}(h)$ for all $1 \leq k \leq n$ and hence $\mathrm{s}(k) \notin \mathbb{E}(\alpha, m e)$ for any $\alpha \in \Phi, m \in \mathbb{Z}$. Hence

$$
\mathrm{s} \notin \bigcup_{\substack{v \in \mathscr{P}_{n}^{\ell}(h) \\ \mathrm{t} \in \operatorname{Std}(v), \mathrm{t} \neq \mathrm{s}}} \operatorname{Path}^{+}(\lambda, \mathrm{t})
$$

and the result follows.

Example 2.12 Let $h=1$ and $\ell=3$ and $\kappa=(0,1,2) \in(\mathbb{Z} / 4 \mathbb{Z})^{3}$ as in Fig. 2. The unique $\lambda \in \mathcal{F}_{n}^{3}(h)$ is given by $\left.\lambda=\left(\left(1^{3}\right),\left(1^{3}\right),\left(1^{2}\right)\right)\right\}$. The tableau $\mathrm{t}^{\lambda}$ is the unique element of $\operatorname{Std}_{e}(\lambda)$ and hence $D_{8}(\lambda)$ is 1 -dimensional.

\section{The skeleton of our BGG resolutions}

In Subsection 3.1 we construct the homomorphisms between standard and Specht modules which will provide the backbone of our BGG complexes. We then consider how these homomorphisms compose (in terms of "diamonds" and "degenerate diamonds" or "strands") and it is these in-depth diagrammatic calculations that provide the technical crux of the paper: In Subsection 3.2 we classify the diamonds in terms of pairs of reflections in the alcove geometry; in Subsection 3.3 we localise to consider the $\mu$ weight-spaces of cell-modules $\Delta(\lambda)$ for $\mu, \lambda$ two points in a given diamond; and finally in Subsection 3.4 we use these results to prove that, within a diamond, composition of homomorphisms commutes (up to scalar multiplication by \pm 1 ) or is zero (for degenerate diamonds). We refer forward to equation (4.3) and (4.4) for the motivation for the terminology of diamonds and strands.

\subsection{One column homomorphisms}

Let $e>h \ell$.

Given $1 \leq i<j \leq h \ell$ and $\alpha, \beta \in \mathscr{P}_{n}^{\ell}(h)$, we suppose that $\ell(\alpha)=\ell(\beta)-1$ and that $\beta \triangleright \alpha$. Then there exists a unique hyperplane $\mathbb{E}\left(\varepsilon_{i}-\varepsilon_{j}, \mu_{i j} e\right)$ for $1 \leq i, j \leq h \ell$ and $\mu_{i j} \in \mathbb{Z}$ such that $s_{i-j, \mu_{i j} e}(\alpha)=\beta$. By definition, this amounts to removing a series of nodes from the $j$ th column of $\alpha$ and adding them in the $i$ th column of $\alpha$ to obtain $\beta \in \mathscr{P}_{n}^{\ell}$ or vice versa. By not assuming that $i<j$, we can use the notation

$$
s_{i-j, \mu_{i j} e}(\alpha)=\beta
$$

to always mean that $\beta$ is obtained by removing a series of nodes from the $j$ th column of $\alpha$ and adding them in the $i$ th column of $\beta$. There are two distinct cases to consider. 
The most familiar case (to many Lie theorists) is that in which $\ell_{\varepsilon_{i}-\varepsilon_{j}}(\alpha, \beta)=1$. In other words $\mu_{i j} \in \mathbb{Z}$ is the unique value such that

$$
\alpha \in \mathbb{E}^{>}\left(\varepsilon_{i}-\varepsilon_{j}, \mu_{i j} e\right) \quad \beta \in \mathbb{E}^{<}\left(\varepsilon_{i}-\varepsilon_{j}, \mu_{i j} e\right)
$$

We refer to such pairs $(\alpha, \beta)$ as maximal pairs. These pairs include those related by a reflection "through a common alcove wall". The other case (which should be familiar to those who study blob and Virasoro algebras) is that in which

$$
\ell_{\varepsilon_{i}-\varepsilon_{j}}(\alpha, \beta)=2 \ell_{\varepsilon_{i}-\varepsilon_{j}}(\alpha)-1
$$

and so $\mathbb{E}\left(\varepsilon_{i}-\varepsilon_{j}, \mu_{i j} e\right)$ is just one of many hyperplanes lying between $\alpha$ and $\beta$; these pairs $(\alpha, \beta)$ correspond to pairs which are as far away as possible in the alcove geometry. We refer to such pairs $(\alpha, \beta)$ as minimal pairs (and they only exist for $\ell>1$ ). We wish to distinguish between such pairs. Therefore, for a minimal (respectively maximal) pair we set $m_{i j}:=\mu_{i j}$ (respectively $M_{i j}:=\mu_{i j}$ ). We have that $m_{i j} \in\{0,1\}$ for any pair $\alpha, \beta \in \mathscr{P}_{n}^{\ell}(h)$.

Example 3.1 Let $h=1, \ell=3$ and $\kappa=(0,1,2)$ as in Fig. 2. The pair $\left(\left(1^{8}|\varnothing|\right.\right.$ $\left.\varnothing),\left(1^{2}|\varnothing| 1^{6}\right)\right)$ is a minimal pair. There are three hyperplanes parallel to $\mathbb{E}_{\varepsilon_{1}-\varepsilon_{3}, 0}$ separating these two points.

Remark 3.2 Note that, near the origin, it is possible that a reflection is both maximal and minimal. For example, consider the pair $\left(1^{6}|\varnothing| 1^{2}\right)$ and $\left(1^{4}|\varnothing| 1^{4}\right)$ pictured in Fig. 2.

Theorem 3.3 Let $e>h \ell$ and suppose that $\kappa \in \mathbb{Z}^{\ell}$ is h-admissible. Let $\alpha \in w_{\alpha} \mathcal{F}_{n}^{\ell}(h)$, $\beta \in w_{\beta} \mathcal{F}_{n}^{\ell}(h)$ be such that $w_{\beta} \leq w_{\alpha}$ and $\ell(\beta)=\ell(\alpha)-1$. Then there exists $1 \leq i, j \leq$ hl and $\mu_{i j} \in \mathbb{N}$ be such that $s_{i-j, \mu_{i j} e}(\alpha)=\beta$. We have that

$$
\alpha \backslash \alpha \cap \beta=\left\{X_{1}, X_{2}, \ldots, X_{k}\right\} \text { and } \beta \backslash \alpha \cap \beta=\left\{Y_{1}, Y_{2}, \ldots, Y_{k}\right\}
$$

with $X_{a} \triangleright X_{a+1}$ (respectively $Y_{a} \triangleright Y_{a+1}$ ) for $1 \leq a<k$ is a sequence of nodes belonging to the $j$ th column of $\alpha$ (respectively ith column of $\beta$ ). There is a unique $\mathrm{T}_{\beta}^{\alpha} \in \operatorname{SStd}^{+}(\beta, \alpha)$, as follows

$$
\mathrm{T}_{\beta}^{\alpha}(\square)= \begin{cases}\square & \text { if } \square \in \alpha \cap \beta \\ Y_{k} & \text { if } \square=X_{k} .\end{cases}
$$

We have $\operatorname{Hom}_{A_{n}(\kappa)}(\Delta(\alpha), \Delta(\beta))=\mathbb{k}\left\{\varphi_{\beta}^{\alpha}\right\}$ where $\varphi_{\beta}^{\alpha}$ is determined by $\varphi_{\beta}^{\alpha}\left(C_{\mathrm{T}^{\alpha}}\right)=C_{\mathrm{T}_{\beta}^{\alpha}}$. We define $\tan _{\alpha \cap \beta}^{\alpha} \in \operatorname{Std}(\alpha)$ and $\tan _{\alpha \cap \beta}^{\beta} \in \operatorname{Std}(\beta)$ to be the unique standard tableaux of given shape determined by

$$
\mathrm{t}_{\alpha \cap \beta}^{\alpha}(r, c, m)=\mathrm{t}^{\alpha \cap \beta}(r, c, m)=\mathrm{t}_{\alpha \cap \beta}^{\beta}(r, c, m)
$$


for $(r, c, m) \in \alpha \cap \beta$. We have that $\phi_{\beta}^{\alpha}\left(c_{\mathrm{t}_{\alpha \cap \beta}^{\alpha}}\right)=c_{\mathrm{t}_{\alpha \cap \beta}^{\alpha}}$ determines the corresponding unique homomorphism in $\operatorname{Hom}_{H_{n}(\kappa)}\left(S_{n}(\alpha), S_{n}(\beta)\right)$. The homomorphisms $\varphi_{\beta}^{\alpha}$ and $\phi_{\beta}^{\alpha}$ are both of degree $t^{1}$.

Proof For the statement for $A_{n}(\kappa)$ see [6, Corollary 10.12]. By the definition of $\mathrm{t}_{\alpha \cap \beta}^{\alpha}$, we have that $\operatorname{Path}\left(\lambda, \mathrm{t}_{\alpha \cap \beta}^{\alpha}\right)=\emptyset$ unless $\lambda \geqslant \alpha$. Therefore $e\left(\mathrm{t}_{\alpha \cap \beta}^{\alpha}\right) \Delta(\lambda)=0$ unless $\lambda \triangleq \alpha$. Therefore $c_{\mathrm{t}_{\alpha \cap \beta}^{\alpha}} \in L(\alpha)$ and thus it is enough to define a homomorphism, $\phi_{\beta}^{\alpha}$ say, by where it sends $c_{\mathrm{t}_{\alpha \cap \beta}^{\alpha}}$. Now, we have that

$$
C_{\varphi\left(\mathrm{t}_{\alpha \cap \beta}^{\alpha}\right)} C_{\mathrm{T}_{\beta}^{\alpha}}=C_{\varphi\left(\mathrm{t}_{\alpha \cap \beta}^{\beta}\right)} \in \mathbf{A}_{n}(\kappa)
$$

and so the result follows by applying the Schur idempotent.

We now consider the restriction of one-column homomorphisms. This will be useful in our inductive proofs. Before stating the proposition, we summarise some trivial consequences of equation (1.4) in the language of reflections. Let $\alpha \in w_{\alpha} \mathcal{F}_{n}^{\ell}(h), \beta \in$ $w_{\beta} \mathcal{F}_{n}^{\ell}(h)$ be such that $w_{\beta} \leq w_{\alpha}$ and $\ell(\beta)=\ell(\alpha)-1$. Let $1 \leq i, j \leq h \ell$ and $\mu_{i j} \in \mathbb{N}$ be such that $s_{i-j, \mu_{i j} e}(\alpha)=\beta$. There exists a unique $\square_{\alpha} \in(\alpha \backslash \beta \cap \alpha) \cap \operatorname{Rem}_{r}(\alpha)$ and $\square_{\beta} \in(\beta \backslash \alpha \cap \beta) \cap \operatorname{Rem}_{r}(\beta)$ for some $r \in \mathbb{Z} / e \mathbb{Z}$. For such a pair, we have that $s_{i-j, \mu_{i j} e}\left(\alpha-\square_{\alpha}\right)=\beta-\square_{\beta}$. Moreover, we have that $r$-res $\left(\left(S_{n}(\alpha)\right)\right) \cong S_{n-1}\left(\alpha-\square_{\alpha}\right)$ and $r$-res $\left(\left(S_{n}(\beta)\right)\right) \cong S_{n-1}\left(\beta-\square_{\beta}\right)$ and these restricted modules are isomorphic to each other if and only $\alpha-\square_{\alpha}=\beta-\square_{\beta}$ (or equivalently $\alpha-\square_{\alpha} \in \mathbb{E}\left(\varepsilon_{i}-\varepsilon_{j}, \mu_{i j} e\right)$ ).

Proposition 3.4 Let $e>$ hl and suppose that $\kappa \in \mathbb{Z}^{\ell}$ is h-admissible. Let $\alpha \in$ $w_{\alpha} \mathcal{F}_{n}^{\ell}(h), \beta \in w_{\beta} \mathcal{F}_{n}^{\ell}(h)$ be such that $w_{\beta} \leq w_{\alpha}$ and $\ell(\beta)=\ell(\alpha)-1$. We set $1 \leq i \neq j \leq h \ell$ and $\mu_{i j} \in \mathbb{N}$ to be such that $s_{i-j, \mu_{i j} e}(\alpha)=\beta$. Let $\square_{\alpha} \in(\alpha \backslash \alpha \cap \beta) \cap \operatorname{Rem}_{r}(\alpha)$ and $\square_{\beta} \in(\beta \backslash \alpha \cap \beta) \cap \operatorname{Rem}_{r}(\beta)$ for some $r \in \mathbb{Z} / e \mathbb{Z}$. If $\alpha-\square_{\alpha} \neq \beta-\square_{\beta}$, then

$$
r-\operatorname{res}\left(\phi_{\beta}^{\alpha}\right)=\phi_{\beta-\square_{\beta}^{\alpha}}^{\alpha-\square_{\alpha}} \in \operatorname{Hom}_{H_{n-1}(\kappa)}\left(S_{n-1}\left(\alpha-\square_{\alpha}\right), S_{n-1}\left(\beta-\square_{\beta}\right)\right)
$$

and if $\alpha-\square_{\alpha}=\beta-\square_{\beta}$ is equal to $\lambda$ say, then

$$
r-\operatorname{res}\left(\phi_{\beta}^{\alpha}\right)=\operatorname{id}_{\lambda}\langle 1\rangle \in \operatorname{Hom}_{H_{n-1}(\kappa)}\left(S_{n-1}(\lambda), S_{n-1}(\lambda)\langle 1\rangle\right)
$$

where $\mathrm{id}_{\lambda}$ is the trivial endomorphism of $S_{n-1}(\lambda)$.

Proof We have already seen (in Theorem 3.3) that the homomorphism $\phi_{\beta}^{\alpha}$ is simply given by right multiplication by $C_{\mathrm{T}_{\beta}^{\alpha}}$. This allows us to factorise the homomorphism in the form

$$
\phi_{\beta}^{\alpha}:\left(\iota_{n-1}^{r}\left(C_{\mathrm{S}}\right) \times C_{\mathrm{T}_{\square_{\alpha}}}\right) \mapsto\left(\iota_{n-1}^{r}\left(\phi_{\beta-\square_{\beta}}^{\alpha-\square_{\alpha}}\left(C_{\mathrm{S}}\right)\right) \times C_{\mathrm{T}_{\square_{\beta}}}\right)
$$

and, ignoring the leftmost strand (as in (1.5)), we obtain the $H_{n-1}(\kappa)$-module homomorphism $\phi_{\beta-\square_{\beta}}^{\alpha-\square_{\alpha}}$. We have that $\operatorname{deg}\left(\mathrm{T}_{\square_{\beta}}\right)=\delta_{\alpha-\square_{\alpha}, \beta-\square_{\beta}}$ and the result follows. 


\subsection{Diamonds formed by pairs of one-column morphisms}

We wish to consider all possible ways of composing a pair of such one-column homomorphisms.

We let $\alpha, \beta \in \mathbb{E}_{h, \ell}^{\odot}$ and we write $\beta \in w_{\beta} \mathcal{F}_{h}(n)$ and $\alpha \in w_{\alpha} \mathcal{F}_{h}(n)$. For the remainder of this section we will assume that $w_{\beta} \leq w_{\alpha}$ with $\ell(\beta)+2=\ell(\alpha)$. We have that $w_{\alpha}=$ $s_{i_{1}} \ldots s_{i_{\ell}}$ for some $s_{i_{k}} \in \mathcal{S}$ for $1 \leq k \leq \ell$ and $w_{\beta}=s_{i_{1}} \ldots \widehat{s}_{i_{p}} \ldots \widehat{s}_{i_{q}} \ldots s_{i_{\ell}}$ for some $1 \leq p, q \leq \ell$, where the hat denotes a generator missing from the product. Therefore there exist precisely two points $\gamma \in w_{\gamma} \mathcal{F}_{h}(n)$ and $\delta \in w_{\delta} \mathcal{F}_{h}(n)$ such that $w_{\beta} \leq$ $w_{\gamma}, w_{\delta} \leq w_{\alpha}$ and, without loss of generality, we have that $w_{\gamma}=s_{i_{1}} \ldots \widehat{s}_{i_{q}} \ldots s_{i_{\ell}}$ and $w_{\delta}=s_{i_{1}} \ldots \widehat{s}_{i_{p}} \ldots s_{i_{\ell}}$. By assumption $\alpha, \beta \in \mathbb{E}_{h, \ell}^{\odot}$, therefore at least one of $\gamma$ or $\delta$ belongs to $\mathbb{E}_{h, \ell}^{\odot}$. We say that such a quadruple $(\alpha, \beta, \gamma, \delta)$ is a diamond if $\alpha, \beta, \gamma, \delta \in \mathbb{E}_{h, \ell}^{\odot}$. If $(\alpha, \beta, \gamma, \delta)$ is not a diamond, then without loss of generality we have that $\delta \notin \mathbb{E}_{h, \ell}^{\odot}$; in which case we say that the triple $(\alpha, \beta, \gamma)$ is a degenerate diamond or a strand. When we wish to speak of both cases simultaneously, we refer to the pair $(\alpha, \beta)$ as a (degenerate or non-degenerate) diamond pair.

The above quadruples $(\alpha, \beta, \gamma, \delta)$ can be broken up into 6 families, for the sake of the upcoming proofs. The first five cases of homomorphisms should be familiar to those who have worked with Carter-Payne homomorphisms for quantum general linear groups (for which $W_{0} \leq W_{e}$ is the maximal parabolic). The sixth case will only occur when the parabolic $W_{0} \leq W_{e}$ is non-maximal.

The pair $\alpha$ and $\beta$ lie in $\mathbb{R}^{3}$. We first consider the cases in which $\alpha, \beta$ differ in precisely three columns. In other words, $\alpha, \beta$ belong to a plane $\mathbb{R}\left\{\varepsilon_{j}-\varepsilon_{i}, \varepsilon_{k}-\varepsilon_{i}\right\}$ for some $1 \leq i, j, k \leq h \ell$ and (without loss of generality) we can assume that

$$
\left\langle\alpha, \varepsilon_{i}\right\rangle>\left\langle\alpha, \varepsilon_{j}\right\rangle>\left\langle\alpha, \varepsilon_{k}\right\rangle
$$

(1) We have $\beta:=s_{k-i, \mu_{k i} e} s_{j-i, \mu_{j i} e}(\alpha)$ and $\gamma:=s_{j-i, \mu_{j i} e}(\alpha)$. Without loss of generality, there are two subcases

(a) $\delta:=s_{k-j, \mu_{k j} e}(\alpha) \in \mathbb{E}_{h, \ell}^{\ominus}$;

(b) $\delta:=s_{k-j, \mu_{k j} e}(\alpha) \notin \mathbb{E}_{h, \ell}^{\ominus}$;

(2) We have $\beta:=s_{k-i, \mu_{k i} e} s_{k-j, \mu_{k j} e}(\alpha)$ and $\gamma:=s_{k-j, \mu_{k j} e}(\alpha)$. Without loss of generality, there are two subcases

(a) $\delta:=s_{j-i, \mu_{j i} e}(\alpha) \in \mathbb{E}_{h, \ell}^{\odot}$;

(b) $\delta:=s_{j-i, \mu_{j i} e}(\alpha) \notin \mathbb{E}_{h, \ell}^{\circ}$;

(3) $\delta:=s_{j-i, \mu_{j i} e}(\alpha)$ and $\gamma:=s_{k-i, \mu_{k i} e}(\alpha) \beta:=s_{k-j, \mu_{k j} e}(\delta)=s_{j-i, \mu_{j i} e}(\gamma)$, all belong to $\mathbb{E}_{h, \ell}^{\ominus}$;

(4) $\delta:=s_{k-j, \mu_{k j} e}(\alpha)$, and $\gamma:=s_{k-i, \mu_{k i} e}(\alpha) \beta:=s_{j-i, \mu_{j i} e}(\delta)=s_{k-j, \mu_{k j}}(\gamma)$, all belong to $\mathbb{E}_{h, \ell}^{\odot}$.

These first four cases can be pictured by projecting into the plane $\mathbb{R}\left\{\varepsilon_{j}-\varepsilon_{i}, \varepsilon_{k}-\varepsilon_{i}\right\}$ as depicted in Fig. 3. 

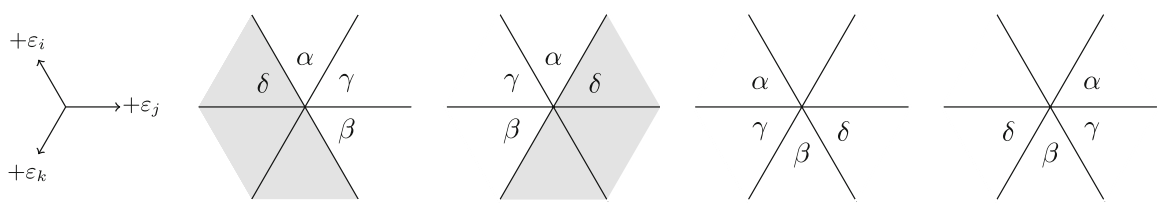

Fig. 3 The cases (1b), (2b), (3) and (4) respectively. We have shaded the non-dominant region in grey. Cases $(1 a)$ and $(2 a)$ can be pictured by removing the shading from $(1 b)$ and $(2 b)$

The pair $\alpha$ and $\beta$ lie in $\mathbb{R}^{4}$. We now assume that $\alpha$ and $\beta$ differ in four columns (so that we cannot picture them belonging to a plane). Without loss of generality, we assume that

$$
\left\langle\alpha, \varepsilon_{i}\right\rangle>\left\langle\alpha, \varepsilon_{j}\right\rangle \quad\left\langle\alpha, \varepsilon_{k}\right\rangle>\left\langle\alpha, \varepsilon_{l}\right\rangle
$$

This is the case in which

(5) $\gamma:=s_{j-i, \mu_{j i} e}(\alpha), \delta:=s_{l-k, \mu_{l k} e}(\alpha)$, and $\beta:=s_{l-k, \mu_{l k} e}(\gamma)=s_{j-i, \mu_{j i} e}(\delta)$ all belong to $\mathbb{E}_{h, \ell}^{\ominus}$.

The pair $\alpha$ and $\beta$ lie in $\mathbb{R}^{2}$. Finally, we have one additional case to consider in which $\alpha$ and $\beta$ differ only in 2 columns. In other words $\alpha$ and $\beta$ belong to a line $\mathbb{R}\left\{\varepsilon_{i}-\varepsilon_{j}\right\}$.

(6a) We have $\beta=s_{i-j,\left(1-m_{j i}\right) e} s_{j-i, m_{j i} e}(\alpha)$ and $\gamma:=s_{j-i, M_{j i}}(\alpha)$ belong to $\mathbb{E}_{h, \ell}^{\odot}$ and

(i) $\delta:=s_{j-i, m_{j i}}(\alpha)$ does not belong to $\mathbb{E}_{h, \ell}^{\odot}$;

(ii) For $\ell>1$ we have that $\delta:=s_{j-i, m_{j i} e}(\alpha)$ does belong to $\mathbb{E}_{h, \ell}^{\odot}$;

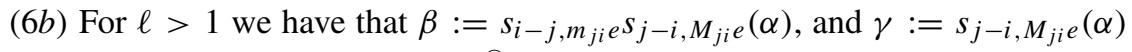
and $\delta:=s_{j-i, m_{j i} e}(\alpha)$ belong to $\mathbb{E}_{h, \ell}^{\odot}$ and cannot be written in the form specified in case $(6 a)$.

In the first two cases, the lightly coloured-in region denotes the "missing" region of $(1 b)$ and $(2 b)$. For diamonds formed entirely of maximal pairs, the pictures in Fig. 3 consists only of six $e$-alcoves and their walls; thus the hyperplanes pictured are the only hyperplanes between $\alpha$ and $\beta$. See Fig. 2 and Eq. (3.6) for such an example. For (degenerate) diamonds involving one or two minimal pairs, there can be many other hyperplanes between $\alpha$ and $\beta$ which are not pictured. See Fig. 2 and Eq. (3.5) for such an example. The fifth case arises from a pair of orthogonal reflections and cannot be pictured in 2-dimensional space, however it is also the easiest case and so we do not lose much by being unable to picture it. The subcases of (6) for which $\ell>1$ are easily pictured and should be familiar to those who work with Virasoro and blob algebras. See Fig. 2 and Eq. (3.4) for such an example (many further examples can be found in [7]). 
Example 3.5 Let $h=1$ and $\ell=3$ and $\kappa=(0,1,2)$ as in Fig. 2. The diamond consisting of

$$
\alpha=\left(1^{8}|\varnothing| \varnothing\right) \quad \beta=\left(1^{2}|\varnothing| 1^{6}\right) \quad \gamma=\left(1^{6}|\varnothing| 1^{2}\right) \quad \delta=\left(1^{2}|\varnothing| 1^{6}\right) .
$$

is as in case $(6 a)$. The diamond consisting of the 3-partitions,

$$
\alpha=\left(\varnothing\left|1^{8}\right| \varnothing\right) \quad \beta=\left(1^{6}|1| 1\right) \quad \gamma=\left(1^{7}|\varnothing| 1\right) \quad \delta=\left(\varnothing\left|1^{7}\right| 1\right) .
$$

is as in case (4) and is a mixture of minimal and maximal pairs. Let

$$
\alpha=\left(\varnothing|\varnothing| 1^{8}\right) \quad \beta=\left(1^{2}|1| 1^{5}\right) \quad \gamma=\left(\varnothing\left|1^{3}\right| 1^{5}\right) \quad \delta=\left(1^{2}|\varnothing| 1^{6}\right) .
$$

The diamond $(\alpha, \beta, \gamma, \delta)$ is as in case (4) and consists solely of maximal pairs.

Remark 3.6 We added in (6b) the condition that it "cannot be written in the form $(6 a)$ " in order to ensure that these cases are mutually exclusive. Without that clause, these cases would have a non-trivial intersection for points near the origin (see Remark 3.2). We have added this clause as these two subcases are genuinely different, see Proposition 3.9 below.

Definition 3.7 Let $(\alpha, \beta, \gamma, \delta)$ be a diamond. We define the $(\alpha, \beta)$-vertex to be

$$
\xi=(\alpha \cap \beta \cap \gamma \cap \delta) \in \mathscr{P}_{n}^{\ell}(h)
$$

In case (6), there exists $0<y<e$ and $x \geq 0$ such that $\left\langle\alpha-\xi, \varepsilon_{i}\right\rangle=x e+y$. In cases (1), (2) and (4), we let $W_{\xi}$ denote the copy of $\mathfrak{S}_{3}$ generated by the reflections through the hyperplanes $\mathbb{E}\left(j-i, \mu_{j i} e\right), \mathbb{E}\left(k-j, \mu_{k j} e\right)$, and $\mathbb{E}\left(k-i, \mu_{k i} e\right)$. Given $s \in W_{\xi}$ we let

$$
x=\left\langle\alpha-\xi, \varepsilon_{i}\right\rangle=\left\langle s(\alpha)-\xi, \varepsilon_{s(i)}\right\rangle \quad y=\left\langle\alpha-\xi, \varepsilon_{j}\right\rangle=\left\langle s(\alpha)-\xi, \varepsilon_{s(j)}\right\rangle
$$

We let $\left\{X_{1}^{s(\alpha)}, X_{2}^{s(\alpha)}, \ldots, X_{x}^{s(\alpha)}\right\}$ denote the final $x$ nodes of the $s(i)$ th column of $s(\alpha)$ and let $\left\{Y_{1}^{s(\alpha)}, Y_{2}^{s(\alpha)}, \ldots, Y_{y}^{s(\alpha)}\right\}$ denote the final $y$ nodes of the $s(j)$ th column of $s(\alpha)$.

Remark 3.8 In cases (1), (2) and (4) we have that $0<y<e$ and $\operatorname{res}\left(X_{k}\right)=\operatorname{res}\left(Y_{k}\right)$ for $1 \leq k \leq \min \{x, y\}$.

\subsection{Paths in diamonds}

We shall now consider reflections of the corresponding paths in the hyperplanes described in our 6 cases above. We remark that each of these paths passes through each hyperplane at most once. Therefore, we simplify our notation of Remark 2.5 by 
dropping the superscript on the reflection. We now consider the (dominant) paths in $\operatorname{Path}(\beta, \alpha)$. In case $(1 a)$ there at two paths

$$
\mathrm{S}_{\beta}^{\alpha}:=s_{j-k, \mu_{k j} e} s_{k-i, \mu_{k i} e}\left(\mathrm{~T}^{\alpha}\right) \text { and } \mathrm{T}_{\beta}^{\alpha}:=s_{j-i, \mu_{j i} e} s_{k-j, \mu_{k j} e}\left(\mathrm{~T}^{\alpha}\right)
$$

of degrees 0 and 2 respectively, which are both dominant. Generic examples of such paths (drawn from the point at which they meet the hyperplane $\mathbb{E}\left(k-j, \mu_{k j} e\right)$ onwards) are pictured below
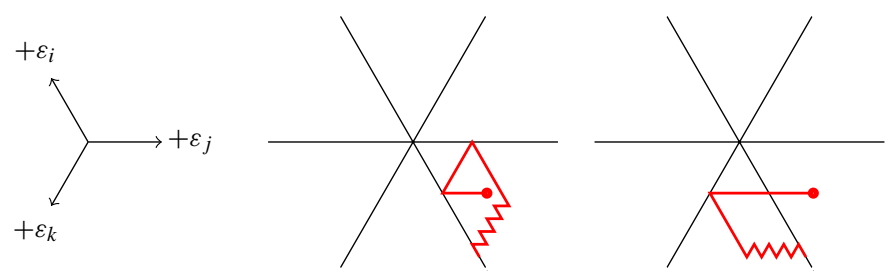

and are of degree 0 and 2 respectively.

In case $(2 a)$ there is a unique path

$$
\mathrm{T}_{\beta}^{\alpha}:=s_{k-i, \mu_{k i}} s_{k-j, \mu_{k j}}\left(\mathrm{~T}^{\alpha}\right)
$$

which is of degree 2 and dominant. A generic example of such a path is pictured below.
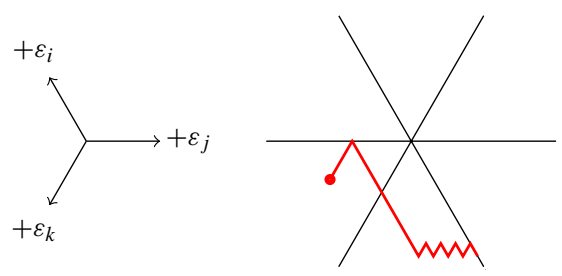

In each of cases $(1 b)$ and $(2 b)$ there is a single path

$$
s_{j-k, \mu_{j k} e} s_{k-i, \mu_{k i} e}\left(\mathrm{~T}^{\alpha}\right) \text { and } s_{j-i, \mu_{j i} e} s_{k-i, \mu_{k i} e}\left(\mathrm{~T}^{\alpha}\right)
$$

of degree 2, neither of which is dominant. These are pictured below
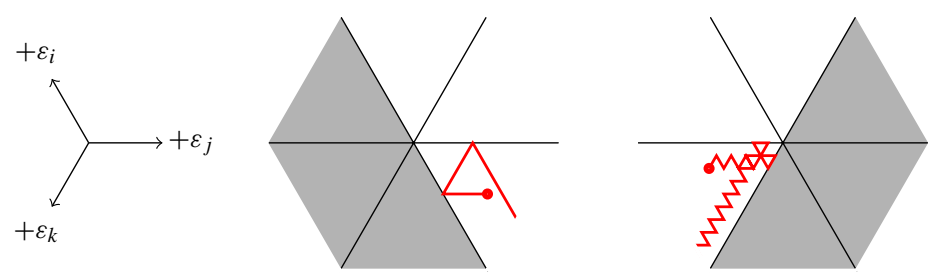
In each of cases (3) and (4) there is a unique path

$$
\mathrm{T}_{\beta}^{\alpha}:=s_{k-j, \mu_{k j} e} s_{j-i, \mu_{j i} e}\left(\mathrm{~T}^{\alpha}\right) \quad \mathrm{T}_{\beta}^{\alpha}:=s_{j-i, \mu_{i j} e} s_{k-j, \mu_{k j} e}\left(\mathrm{~T}^{\alpha}\right)
$$

respectively, of degree 2. Generic examples of such paths are pictured below
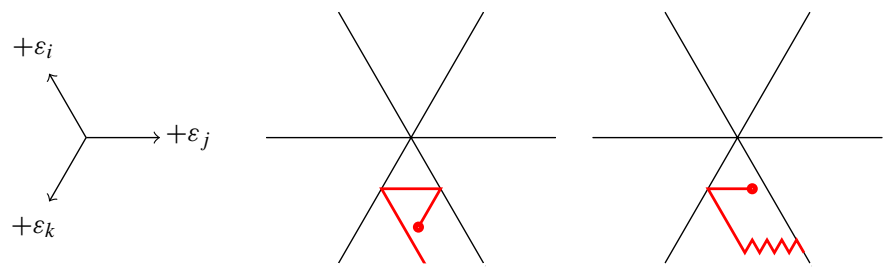

In case (5), the reflections are orthogonal and there is a unique (dominant) path and if we assume (without loss of generality) that $\left\langle\alpha, \varepsilon_{i}\right\rangle>\left\langle\alpha, \varepsilon_{k}\right\rangle$, then this path is given by

$$
\mathrm{T}_{\beta}^{\alpha}:=s_{l-k, \mu_{l k} e} s_{j-i, \mu_{j i} e}\left(\mathrm{~T}^{\alpha}\right)
$$

and is of degree 2 . In case $(6 a)$ we have $(x-1)$ distinct dominant paths of degree 0 given as follows,

$$
\mathrm{S}_{\chi}= \begin{cases}s_{i-j,\left(M_{i j}-\chi-1\right) e} s_{i-j,\left(M_{i j}-\chi\right) e}\left(\mathrm{~T}^{\alpha}\right) & \text { for } m_{i j}=1 \\ s_{i-j,\left(M_{i j}+\chi+1\right) e} s_{i-j,\left(M_{i j}+\chi\right) e}\left(\mathrm{~T}^{\alpha}\right) & \text { for } m_{i j}=0\end{cases}
$$

for $1 \leq \chi<x$ (for $x$ as in Definition 3.7); we also have a unique path of degree 2 given by

$$
\mathrm{T}_{\beta}^{\alpha}=s_{j-i,\left(1-m_{j i}\right) e} s_{i-j, m_{i j} e}\left(\mathrm{~T}^{\alpha}\right) \in \operatorname{Path}\left(\beta, \mathrm{T}^{\alpha}\right)
$$

which is dominant if and only if we are in case $(6 a)(i i)$. In case $(6 b)$ we have a unique (dominant) path

$$
\mathrm{T}_{\beta}^{\alpha}=s_{i-j,\left(2 m_{j i}-M_{j i}\right) e s_{j-i, m_{j i}}\left(\mathrm{~T}^{\alpha}\right)}
$$

of degree 2. Using Eq. (2.2), we now summarise the above as follows.

Proposition 3.9 Let $(\alpha, \beta)$ be a diamond pair. We have that

$$
\operatorname{dim}_{t}\left(1_{\alpha} \Delta(\beta)\right)= \begin{cases}0 & \text { in cases }(1 b) \text { and }(2 b) \\ t^{2}+1 & \text { in case }(1 a) \\ t^{2} & \text { in cases }(2 a),(3),(4),(5) \text { and }(6 b) \\ x-1 & \text { in case }(6 a)(i) \\ t^{2}+x-1 & \text { in case }(6 a)(i i)\end{cases}
$$


where $x \in \mathbb{Z}_{>0}$ is defined in Definition 3.7 and $t$ is the grading indeterminate over $\mathbb{Z}_{\geq 0}$ from Remark 1.16.

Example 3.10 In Fig. 2, we have that the tableaux

$$
\begin{aligned}
& \mathrm{T}_{\left(1^{4}|\varnothing| 1^{4}\right)}^{\left(1^{8}|\varnothing| \varnothing\right)}=s_{1-3, M_{13}}^{(6)} \circ s_{3-1, M_{31}}^{(2)}\left(\mathrm{T}^{\left(1^{8}|\varnothing| \varnothing\right)}\right) \quad \mathrm{T}_{\left(1^{4}|\varnothing| 1^{4}\right)}^{\left(1^{8}|\varnothing| \varnothing\right)}=s_{3-1, m_{31}}^{(7)} \circ s_{1-2, M_{12}}^{(1)}\left(\mathrm{T}^{\left(1^{8}|\varnothing| \varnothing\right)}\right) \\
& \text { and } \quad \mathrm{T}_{\left(1^{4}|\varnothing| 1^{4}\right)}^{\left(1^{8}|\varnothing| \varnothing\right)}=s_{1-2, m_{12}}^{(6)} \circ s_{3-2, m_{32}}^{(5)}\left(\mathrm{T}^{\left(1^{8}|\varnothing| \varnothing\right)}\right)
\end{aligned}
$$

are as in cases $(6 a),(4)$, and (4) respectively and are all of degree $t^{2}$.

\subsection{Compositions of one-column homomorphisms in diamonds}

We now consider the composition of the one-column homomorphisms in terms of the path basis constructed in Proposition 3.9. Let $\mathrm{T} \in \mathcal{T}(\lambda, \mu)$ and $\mathrm{T}(X)=\mathbf{I}_{Y}^{\kappa} \in \mathbb{Z}[\epsilon]$ for $X \in \lambda, Y \in \mu$; we abuse notation by writing either $\mathrm{T}(X)=Y$ or $\mathrm{T}(X)=\mathbf{I}_{Y}^{\kappa}$. From Proposition 3.9, we deduce the immediate corollary.

Corollary 3.11 Let $(\alpha, \beta, \gamma)$ be a strand (in other words, as in cases (1b), (2b) and $(6 a)(i))$. We have that $\varphi_{\gamma}^{\alpha} \circ \varphi_{\beta}^{\gamma}=0 \in \operatorname{Hom}_{A_{n}(\kappa)}(\Delta(\alpha), \Delta(\beta))$.

Proof Cases (1b) and (2b) are clear. Case (6a)(i) follows because the composition of two homomorphism of degree $t^{1}$ must be a vector of degree $t^{2}$ and no such vector exists (by Proposition 3.9).

Proposition 3.12 Let $(\alpha, \beta, \gamma, \delta)$ be a diamond. We have that

- $C_{\mathrm{T}_{\beta}^{\alpha}}=C_{\mathrm{T}_{\delta}^{\alpha}} C_{\mathrm{T}_{\beta}^{\delta}}$ in all cases, namely (1a), (2a), (3), (4), (5), (6a)(i) and (6b);

- $C_{\mathrm{T}_{\beta}^{\alpha}}=C_{\mathrm{T}_{\gamma}^{\alpha}} C_{\mathrm{T}_{\beta}^{\gamma}}$ in the cases (2a), (3), (5).

Proof For $\gamma \in \mathscr{P}_{n}^{\ell}$ (similarly for $\delta \in \mathscr{P}_{n}^{\ell}$ ) it is clearly enough to show that

$$
\mathrm{T}_{\gamma}^{\alpha} \mathrm{T}_{\beta}^{\gamma}=\mathrm{T}_{\beta}^{\alpha} \in \mathcal{T}(\beta, \alpha)
$$

on the level of bijective maps : $\beta \rightarrow \alpha$, and furthermore that if

$$
\begin{aligned}
& (r, c, m) \triangleright\left(r^{\prime}, c^{\prime}, m^{\prime}\right) \text { and } \mathrm{T}_{\beta}^{\gamma}(r, c, m) \triangleleft \mathrm{T}_{\beta}^{\gamma}\left(r^{\prime}, c^{\prime}, m^{\prime}\right) \text { implies } \\
& \quad \mathrm{T}_{\gamma}^{\alpha} \mathrm{T}_{\beta}^{\gamma}(r, c, m) \triangleleft \mathrm{T}_{\gamma}^{\alpha} \mathrm{T}_{\beta}^{\gamma}\left(r^{\prime}, c^{\prime}, m^{\prime}\right)
\end{aligned}
$$

for any two nodes $(r, c, m),\left(r^{\prime}, c^{\prime}, m^{\prime}\right) \in \beta \backslash \xi$ of the same or adjacent residue. This is simply by the definition of the bases elements corresponding to these tableaux (and the fact that double-crossings between strands of non-adjacent distinct residues can be removed by relation (A5)). The cases listed in the above proposition are precisely those for which Eqs. (3.7) and (3.8) are both true (in other words, 3.7 and 3.8 both hold in all cases except in cases (1a), (4), and (6) for the product $\mathrm{T}_{\gamma}^{\alpha} \mathrm{T}_{\beta}^{\gamma}$ - which will be discussed separately). 
We shall consider case (2), as the other cases are identical. It is clear that $\mathrm{S}(r, c, m)=(r, c, m)$ if $(r, c, m) \in \xi$ for $\mathrm{T}_{\mu}^{\lambda}$ for $\lambda, \mu \in\{\alpha, \beta, \gamma, \delta\}$. Thus it remains to consider the restriction of these bijections to : $\beta \backslash \xi \rightarrow \alpha \backslash \xi$ (via both $\gamma \backslash \xi$ and $\delta \backslash \xi)$. We have that

$$
\begin{aligned}
& \mathrm{T}_{\beta}^{\gamma}\left(X_{p}^{\beta}\right)=Y_{p}^{\gamma} \quad \mathrm{T}_{\beta}^{\gamma}\left(X_{y+q}^{\beta}\right)=X_{y+q}^{\gamma} \quad \mathrm{T}_{\beta}^{\gamma}\left(Y_{p}^{\beta}\right)=X_{p}^{\gamma} \\
& \mathrm{T}_{\gamma}^{\alpha}\left(Y_{p}^{\gamma}\right)=Y_{p}^{\alpha} \quad \mathrm{T}_{\gamma}^{\alpha}\left(X_{y+q}^{\gamma}\right)=X_{y+q}^{\alpha} \quad \mathrm{T}_{\gamma}^{\alpha}\left(X_{p}^{\gamma}\right)=X_{p}^{\alpha}
\end{aligned}
$$

and

$$
\begin{aligned}
& \mathrm{T}_{\beta}^{\delta}\left(X_{p}^{\beta}\right)=X_{p}^{\delta} \quad \mathrm{T}_{\beta}^{\delta}\left(X_{y+q}^{\beta}\right)=X_{y+q}^{\delta} \quad \mathrm{T}_{\beta}^{\delta}\left(Y_{p}^{\beta}\right)=Y_{p}^{\delta} \\
& \mathrm{T}_{\delta}^{\alpha}\left(X_{p}^{\delta}\right)=Y_{p}^{\alpha} \quad \mathrm{T}_{\delta}^{\alpha}\left(X_{y+q}^{\delta}\right)=X_{y+q}^{\alpha} \quad \mathrm{T}_{\delta}^{\alpha}\left(Y_{p}^{\beta}\right)=X_{p}^{\alpha}
\end{aligned}
$$

and

$$
\mathrm{T}_{\beta}^{\alpha}\left(X_{p}^{\beta}\right)=Y_{p}^{\alpha} \quad \mathrm{T}_{\beta}^{\alpha}\left(X_{y+q}^{\beta}\right)=X_{y+q}^{\alpha} \quad \mathrm{T}_{\beta}^{\alpha}\left(Y_{p}^{\beta}\right)=X_{p}^{\alpha}
$$

for $1 \leq p \leq y$ and $1 \leq q \leq x-y$. Therefore equation (3.7) holds. To see that equation (3.8) holds, one requires the following observation

$$
X_{j}^{\beta} \triangleright Y_{j}^{\beta} \quad Y_{j}^{\gamma} \triangleright X_{j}^{\gamma} \quad X_{j}^{\delta} \triangleright Y_{j}^{\delta} \quad Y_{j}^{\alpha} \triangleright X_{j}^{\alpha}
$$

for all $1 \leq j \leq y$; one can apply this observation to each of the above tableaux in turn. Thus equation (3.8) holds, as required.

It remains to consider the $\gamma$ subcases of $(1 a)$, (4), and (6) not considered above. In all these cases, we shall see that equation (3.7) and (3.8) fail. Thus, we must apply some relations in order to rewrite each product-diagram in the required form. Given $(r, c, m) \in \alpha$, we let $y(r, c, m) 1_{\alpha}$ denote the diagram $1_{\alpha}$ with a dot added on the vertical solid strand with $x$-coordinate given by $\mathbf{I}_{(r, c, m)}^{\kappa}$. Following [10], we set $y_{k}=\mathrm{y}(k, 1, \ell)$.

Proposition 3.13 Let $(\alpha, \beta, \gamma, \delta)$ be a diamond as in case (1a). Then

$$
C_{\mathrm{T}_{\gamma}^{\alpha}} C_{\mathrm{T}_{\beta}^{\gamma}}=-y\left(X_{y}^{\alpha}\right) 1_{\alpha} C_{\mathrm{S}_{\beta}^{\alpha}}=(-1)^{y+1} C_{\mathrm{T}_{\beta}^{\alpha}}+A_{n}^{\triangleright \beta}(\kappa) .
$$

Proof In case (1a), we have $\mathrm{T}_{\gamma}^{\alpha} \circ \mathrm{T}_{\beta}^{\gamma}=\mathrm{S}_{\beta}^{\alpha} \in \mathcal{T}(\beta, \alpha)$ as bijective maps. However, the corresponding product of diagrams has a single double-crossing of non-zero degree; this is between the strand from $X_{y+1}^{\beta}$ on the southern edge to $X_{y+1}^{\alpha}$ on the northern edge and the strand from $Y_{y}^{\beta}$ on the southern edge to $X_{y}^{\alpha}$ on the northern edge. In particular, $\operatorname{res}\left(X_{y+1}^{\beta}\right)=\operatorname{res}\left(Y_{y}^{\beta}\right)-1$, and $X_{y+1}^{\beta} \triangleleft Y_{y}^{\beta} \quad \mathrm{T}_{\beta}^{\gamma}\left(X_{y+1}^{\beta}\right)=X_{y+1}^{\gamma} \triangleright Y_{y}^{\gamma}=\mathrm{T}_{\beta}^{\gamma}\left(Y_{y}^{\beta}\right) \quad \mathrm{T}_{\gamma}^{\alpha}\left(Y_{y}^{\gamma}\right)=X_{y}^{\alpha} \triangleright X_{y+1}^{\alpha}=\mathrm{T}_{\gamma}^{\alpha}\left(X_{y+1}^{\gamma}\right)$. 
For $1 \leq p, p^{\prime} \leq y$ the strand from $X_{y+p}^{\beta}$ on the southern edge to $X_{y+p}^{\alpha}$ on the northern edge double-crosses with the strand from $Y_{p^{\prime}}^{\beta}$ on the southern edge to $X_{p^{\prime}}^{\alpha}$ on the northern edge; since $y<e$, we can remove all of the double-crossings for $(p, q) \neq(1,1)$ using relation (A5). We now resolve the final double crossing (for $(p, q)=(1,1))$ using relation the leftmost equality of (A6) and hence obtain

$$
C_{\mathrm{T}_{\gamma}^{\alpha}} C_{\mathrm{T}_{\beta}^{\gamma}}=y\left(X_{y+1}^{\alpha}\right) 1_{\alpha} C_{\mathrm{S}_{\beta}^{\alpha}}-y\left(X_{y}^{\alpha}\right) 1_{\alpha} C_{\mathrm{S}_{\beta}^{\alpha}}
$$

Concerning the former diagram: we pull the dot down the strand and encounter no like-crossings on the way; hence this term is equal to zero. It remains to prove the second equality in Eq. (3.9). We let $\mathrm{U}_{y} \in \mathcal{T}(\alpha, \beta)$ denote the map

$$
\mathrm{U}_{y}(r, c, m)=\left\{\begin{array}{ll}
X_{y}^{\alpha} & \text { for }(r, c, m)=X_{y}^{\beta} \\
Y_{y}^{\alpha} & \text { for }(r, c, m)=Y_{y}^{\beta} \\
\mathrm{S}_{\beta}^{\alpha}(r, c, m) & \text { otherwise. }
\end{array} .\right.
$$

We claim that

$$
\mathrm{y}\left(X_{y}^{\alpha}\right) 1_{\alpha} C_{\mathrm{S}_{\beta}^{\alpha}}=-C_{\mathrm{U}_{y}}+A_{n}^{\triangleright \beta}(\kappa) .
$$

To see this, pull the dot at the top of the diagram $y\left(X_{y}^{\alpha}\right) 1_{\alpha} C_{\mathrm{S}_{\beta}^{\alpha}}$ down the strand on which it lies (from $X_{y}^{\alpha}$ on northern edge to $Y_{y}^{\beta}$ on the southern edge) towards the bottom of the diagram. By Definition 1.10, we can do this freely until we encounter a likecrossing of the form in relation (A3). Such a crossing involves the aforementioned strand (between points $X_{y}^{\alpha}$ and $Y_{y}^{\beta}$ on the northern and southern edges) and some vertical strand of the same residue. Such a vertical strand either $(i)$ corresponds to a step of the form $+\varepsilon_{m}$ for $m \notin\{i, j, k\}$ or $(i i)$ is the vertical strand from $X_{y}^{\beta}$ on the southern edge to $Y_{y}^{\alpha}$ on the northern edge. In the former case, the resulting error term belongs to $A_{n}^{\triangleright \beta}(\kappa)$. In the latter case, we apply relation (A3) to move the dot past the crossing at the expense of acquiring an error term, which is equal to $-C_{\cup_{y}}$. Finally (in the diagram which has a dot) we continue pulling the dot reaches the bottom of the diagram, the resulting diagram again belongs to $A_{n}^{\triangleright \beta}(\kappa)$. Thus the only non-zero term acquired in this process is $-C_{\mathrm{U}_{y}}$ and the claim holds. If $y=1$, then $\mathrm{U}_{y}=\mathrm{T}_{\beta}^{\alpha}$ and we are done. Suppose that $y>1$. Consider

(i) the solid strand from $Y_{y-1}^{\beta}$ on the southern edge to $X_{y-1}^{\alpha}$ on the northern edge

(ii) the solid strand from $X_{y-1}^{\beta}$ on the southern edge to $Y_{y-1}^{\alpha}$ on the northern edge (iii) the ghost strand from $Y_{y}^{\beta}$ on the southern edge to $Y_{y}^{\alpha}$ on the northern edge.

These three strands together form a triple-crossing as on the right-hand side of the rightmost equation in relation (A7). Applying relation (A7), we can undo the crossing (at the expense of multiplication by minus one and an error term with the same number of crossings). Consider the error term: We are free to pull the ghost strand (of the strand connecting $Y_{y}^{\beta}$ and $Y_{y}^{\alpha}$ ) to the left to obtain a diagram which belongs to $A_{n}^{\triangleright \beta}(\kappa)$. That 
leaves one remaining non-zero diagram which differs from $-C_{\cup_{y}}$ in that we have undone the aforementioned triple-crossing; to summarise

$\mathrm{y}\left(X_{y}^{\alpha}\right) 1_{\alpha} C_{\mathrm{S}_{\beta}^{\alpha}}=C_{\mathrm{U}_{y-1}}+A_{n}^{\triangleright \beta}(\kappa) \quad$ with $\quad \mathrm{U}_{y}(r, c, m)= \begin{cases}X_{y-1}^{\alpha} & \text { for }(r, c, m)=X_{y-1}^{\beta} \\ Y_{y-1}^{\alpha} & \text { for }(r, c, m)=Y_{y-1}^{\beta} \\ \mathrm{U}_{y}(r, c, m) & \text { otherwise. }\end{cases}$

Repeat this argument until all $y$ crossings have been resolved, the results follows.

Proposition 3.14 Let $(\alpha, \beta, \gamma, \delta)$ be a diamond as in case (4). Then

$$
C_{\mathrm{T}_{\gamma}^{\alpha}} C_{\mathrm{T}_{\beta}^{\gamma}}=(-1)^{x} C_{\mathrm{T}_{\beta}^{\alpha}}+A_{n}^{\triangleright \beta}(\kappa) .
$$

Proof We have that

$$
\mathrm{T}_{\gamma}^{\alpha} \circ \mathrm{T}_{\beta}^{\gamma}(r, c, m)= \begin{cases}X_{p}^{\alpha}=\mathrm{T}_{\beta}^{\alpha}\left(Y_{p}^{\beta}\right) & \text { if }(r, c, m)=X_{p}^{\beta} \text { for } 1 \leq p \leq x \\ Y_{p}^{\alpha}=\mathrm{T}_{\beta}^{\alpha}\left(X_{p}^{\beta}\right) & \text { if }(r, c, m)=Y_{p}^{\beta} \text { for } 1 \leq p \leq x \\ Y_{q}^{\alpha}=\mathrm{T}_{\beta}^{\alpha}\left(Y_{q}^{\beta}\right) & \text { if }(r, c, m)=Y_{q}^{\beta} \text { for } x+1 \leq q \leq y \\ \mathrm{~T}_{\beta}^{\alpha}(r, c, m) & \text { for }(r, c, m) \in \xi\end{cases}
$$

Consider

(i) the solid strand from $Y_{x}^{\beta}$ on the southern edge to $X_{x}^{\alpha}$ on the northern edge;

(ii) the solid strand from $X_{x}^{\beta}$ on the southern edge to $Y_{x}^{\alpha}$ on the northern edge;

(iii) the ghost strand of the strand from $Y_{x+1}^{\beta}$ on the southern edge to $Y_{x+1}^{\alpha}$ on the northern edge.

These strands together form a crossing as on the right hand side of the rightmost equation in relation (A7). Undoing this crossing we obtain an error term (corresponding to the diagram on the lefthand-side of the rightmost equality in relation (A7)) which belongs to $A_{n}^{\triangleright \beta}(\kappa)$ and another (non-zero) term. One can then repeat the above argument with the latter diagram (except replacing the subscript ' $x$ ' with ' $x-1$ '). Continuing in this fashion, we obtain the required result.

Proposition 3.15 Let $(\alpha, \beta, \gamma, \delta)$ be a diamond as in case (6b). Then

$$
C_{\mathrm{T}_{\gamma}^{\alpha}} C_{\mathrm{T}_{\beta}^{\gamma}}=(-1)^{y} C_{\mathrm{T}_{\beta}^{\alpha}}+A_{n}^{\triangleright \beta}(\kappa) .
$$

Proof We let $\left\{X_{1}^{\alpha}, X_{2}^{\alpha}, \ldots, X_{e x}^{\alpha}, Y_{1}^{\alpha}, \ldots, Y_{y}^{\alpha}\right\}$ denote the final $x e+y$ nodes of the $i$ th column of $\alpha$. We let $\left\{X_{1}^{\beta}, X_{2}^{\beta}, \ldots, X_{e x}^{\beta}\right\}$ denote the final $x e$ nodes of the $j$ th column of $\beta$ and $\left\{Y_{1}^{\beta}, \ldots, Y_{y}^{\beta}\right\}$ denote the final $y$ nodes of the $i$ th column of $\beta$. We have that

$$
\mathrm{T}_{\gamma}^{\alpha} \circ \mathrm{T}_{\beta}^{\gamma}(r, c, m)= \begin{cases}\mathrm{T}_{\beta}^{\alpha}\left(Y_{p}^{\beta}\right)=X_{p}^{\alpha} & \text { for }(r, c, m)=X_{p}^{\beta} \text { and } 1 \leq p \leq y \\ \mathrm{~T}_{\beta}^{\alpha}\left(X_{p}^{\beta}\right)=Y_{p}^{\alpha} & \text { for }(r, c, m)=Y_{p}^{\beta} \text { and } 1 \leq p \leq y \\ \mathrm{~T}_{\beta}^{\alpha}\left(X_{q}^{\beta}\right)=X_{q}^{\alpha} & \text { for }(r, c, m)=X_{q}^{\beta} \text { and } y<q \leq e x \\ \mathrm{~T}_{\beta}^{\alpha}(r, c, m) & \text { for }(r, c, m) \in \xi .\end{cases}
$$




\section{Consider}

(i) the solid strand from $Y_{y}^{\beta}$ on the southern edge to $X_{y}^{\alpha}$ on the northern edge;

(ii) the solid strand from $X_{y}^{\beta}$ on the southern edge to $Y_{y}^{\alpha}$ on the northern edge;

(iii) the ghost of the strand from $X_{y+1}^{\beta}$ on the southern edge to $X_{y+1}^{\alpha}$ on the northern edge.

These strands together form a crossing as on the right hand side of the latter equality in relation (A7). Undoing this crossing we obtain an error term (corresponding to the diagram on the lefthand-side of the latter equality in relation (A7)) which belongs to $A_{n}^{\triangleright \beta}(\kappa)$ and another (non-zero) term. One can then repeat the above argument with the latter diagram (except replacing the subscript ' $y$ ' with ' $y-1^{\prime}$ ). Continuing in this fashion, we obtain the required result.

Proposition 3.16 Let $(\alpha, \beta)$ be a diamond pair as in case $(6 a)(i i)$. Then

$$
C_{\mathrm{T}_{\gamma}^{\alpha}} C_{\mathrm{T}_{\beta}^{\gamma}}=(-1)^{e(x+1)+y} C_{\mathrm{T}_{\beta}^{\alpha}}+A_{n}^{\triangleright \beta}(\kappa) .
$$

Proof We first fix some notation. We denote the final $e$ nodes at the end of the $j$ th column of $\beta$ by $X_{1}^{\beta}, \ldots, X_{e}^{\beta}$. We denote the final $e(x-1)+y$ nodes at the end of the $i$ th column of $\beta$ by $X_{e+1}^{\beta}, \ldots, X_{e x}^{\beta}, Y_{1}^{\beta}, \ldots, Y_{y}^{\beta}$. We let $X_{1}^{\alpha}, X_{2}^{\alpha}, \ldots, X_{e x}^{\alpha}, Y_{1}^{\alpha}, \ldots, Y_{y}^{\alpha}$ denote the final $e x+y$ nodes at the end of the $i$ th column of $\alpha$. Given $\sigma \in \mathfrak{S}_{x}$ we define $\mathrm{U}_{\sigma} \in \mathcal{T}(\beta, \alpha)$ as follows,

$$
\mathrm{U}_{\sigma}\left(X_{e p-q}^{\beta}\right)=\mathrm{U}_{\sigma}\left(X_{e \sigma(p)-q}^{\beta}\right) \quad \mathrm{U}_{\sigma}\left(Y_{t}^{\beta}\right)=Y_{t}^{\alpha}
$$

for $1 \leq p, \leq x, 0 \leq q<e, 0 \leq t<y$ and such that $\mathrm{U}_{\sigma}(r, c, m)=(r, c, m)$ for $(r, c, m) \in \xi$. We have that $\mathrm{T}_{\beta}^{\alpha}=\mathrm{U}_{\text {id }}$ for id $\in \mathfrak{S}_{x}$ and $\mathrm{S}_{\chi}=\mathrm{U}_{\sigma}$ for $\sigma=s_{1} s_{2} \ldots s_{\chi}$ for $1 \leq \chi<x$ (and so any element of $\operatorname{SStd}(\beta, \alpha)$ can be written in the form of equation (3.10)).

We now state a claim that will provide the crux of the proof. Set $\sigma=s_{1} s_{2} \ldots s_{\chi}$ for $1 \leq \chi \leq x$. Given $1 \leq r<\chi$, we refer to the strand in $C_{\mathrm{U}_{\sigma}}$ from $X_{e}^{\beta}$ on the southern edge to $X_{\chi e}^{\alpha}$ on the northern as the principal strand. Let $C_{\mathrm{U}_{\sigma}}^{r}$ denote the diagram obtained from $C_{\mathrm{U}_{\sigma}}$ by placing a dot on the principal strand at any point in the interval $\left(\mathbf{I}_{X_{e r}^{\alpha}}^{\kappa}, \mathbf{I}_{X_{e r+1}^{\alpha}}^{\kappa}\right) \times[0,1]$.

For $\sigma \neq s_{1}$, we claim that

$$
\begin{aligned}
& C_{\mathrm{U}_{\sigma}}^{r}=C_{\mathrm{U}_{\sigma}}^{r-1}+(-1)^{e+1} C_{\mathrm{U}_{\sigma^{\prime}}}^{r-1} \\
& C_{\mathrm{U}_{s_{1}}}^{r}=C_{\mathrm{U}_{s_{1}}}^{r-1}+(-1)^{e} C_{\mathrm{U}_{s_{1}}}
\end{aligned}
$$

modulo $A_{n}^{\triangleright \beta}(\kappa)$ where $\sigma^{\prime}=s_{1} s_{2} \ldots s_{r-1} s_{r+1} \ldots s_{\chi}$. Diagrammatically, we can think of our claim as simply a beefed-up version of relation (A3) in which we consider crossings involving collections of strands (each of size $e>1$ ). We let $i=\operatorname{res}\left(X_{e}^{\beta}\right)$.

We now prove the claim. First apply relation (A3) to pull the dot through the crossing $i$-strands and hence obtain $C_{\mathrm{U}_{\sigma}}^{r-1}$ plus another term with a minus sign. For this 
latter diagram, the ghost of the principal $i$-strand can be pulled to the left through the crossing solid $(i+1)$-strands as in relation (A7). We hence obtain two diagrams: one with the same number of crossings, and one in which the crossing of $(i+1)$-strands has been undone. The former is zero modulo the stated ideal. The latter diagram now has a crossing of two solid $(i+2)$-strands and a ghost $(i+1)$-strand as in relation (A7). Repeating as necessary, this process terminates with a diagram (occurring with coefficient $(-1)^{e}$ ) which traces out the bijection of $U_{\sigma^{\prime}}$ but with many double-crossings.

- If $\sigma=s_{1}$, then all of these double-crossings are of degree zero;

- If $\sigma \neq s_{1}$, then precisely one of these double-crossings has non-zero degree: that between the solid strand from $X_{e}^{\beta}$ on the southern edge to $X_{r e}^{\alpha}$ on the northern edge and the ghost of the strand from $X_{r e-e+1}^{\beta}$ on the southern edge to $X_{\chi e-e+1}^{\alpha}$ on the northern edge.

In the latter case, we resolve this double-crossing as in relation (A7) and obtain two diagrams: one is of the required form and the other belongs to the stated ideal. In either case, the claim holds. Having proven our claim, we are now ready to prove the result. We have that

$$
\mathrm{T}_{\gamma}^{\alpha} \circ \mathrm{T}_{\beta}^{\gamma}(r, c, m)= \begin{cases}\mathrm{U}_{s_{1} \ldots s_{x-1}}\left(X_{t}^{\beta}\right)=X_{x e-e+t}^{\alpha} & \text { for }(r, c, m)=Y_{t}^{\beta} \text { and } 1 \leq t \leq y \\ \mathrm{U}_{s_{1} \ldots s_{x-1}}\left(Y_{t}^{\beta}\right)=Y_{t}^{\alpha} & \text { for }(r, c, m)=X_{t}^{\beta} \text { and } 1 \leq t \leq y \\ \mathrm{U}_{s_{1} \ldots s_{x-1}}(r, c, m) & \text { otherwise. }\end{cases}
$$

Therefore, using $y$ applications of (A7) we obtain a diagram which traces out the same bijection as $U_{s_{1} \ldots s_{x-1}}$ (modulo error terms). However the resulting diagram contains a single degree 2 double-crossing between the solid strand from $X_{e}^{\beta}$ to $X_{e x}^{\alpha}$ (on the southern and northern edges, respectively) with the ghost of the strand from $Y_{1}^{\beta}$ to $Y_{1}^{\alpha}$ (on the southern and northern edges, respectively). Resolving this crossing using relation (A6), we obtain that

$$
C_{\mathrm{T}_{\gamma}^{\alpha}} C_{\mathrm{T}_{\beta}^{\gamma}}=(-1)^{y+1} C_{\mathrm{U}_{s_{1} \ldots s_{x-1}}}+A_{n}^{\triangleright \beta}(\kappa) .
$$

We now successively apply equation (3.11) a total of $x-1$ times, followed by a single application of equation (3.12). The error terms all belong to $A_{n}^{\triangleright \beta}(\kappa)$ and the result follows.

Theorem 3.17 Let $(\alpha, \beta, \gamma, \delta)$ be a diamond. We have that

$$
\varphi_{\delta}^{\alpha} \circ \varphi_{\beta}^{\delta}=\varphi_{\beta}^{\alpha}=\varepsilon_{\alpha, \beta, \gamma, \delta} \varphi_{\gamma}^{\alpha} \circ \varphi_{\beta}^{\gamma} \quad \text { where } \varepsilon_{\alpha, \beta, \gamma, \delta}= \begin{cases}(-1)^{y+1} & \text { in cases }(1 a) \\ (-1)^{x} & \text { in cases }(4) \\ (-1)^{y} & \text { in cases }(6 b) \\ (-1)^{e(x+1)+y} & \text { in case }(6 a)(i i) \\ 1 & \text { otherwise } \text {. }\end{cases}
$$


Moreover the map $\varphi_{\beta}^{\alpha}$ is determined by $\varphi_{\beta}^{\alpha}\left(C_{\mathrm{T}^{\alpha}}\right)=C_{\mathrm{T}_{\beta}^{\alpha}}$ and $\operatorname{dim}_{t}\left(\operatorname{Hom}_{\mathbf{A}_{n}(\kappa)}(\boldsymbol{\Delta}(\alpha)\right.$, $\boldsymbol{\Delta}(\beta)))=t^{2}$ where $t$ is the grading indeterminate over $\mathbb{Z}_{\geq 0}$ from Remark 1.16.

Proof Equation (3.13) is simply a restatement of Propositions 3.12 to 3.16; we remark that we can ignore all mention of terms in the ideal $A_{n}^{\triangleright \beta}(\kappa)$ in Propositions 3.14 to 3.16 as all these terms are zero in $\boldsymbol{\Delta}(\beta)$ (by the definition of the cell module as a quotient by precisely this ideal). To verify that the homomorphism space is 1-dimensional, it remains to check that $C_{\mathrm{S}} \in L(\beta)$ for $\mathrm{S} \in \mathrm{SStd}^{+}(\beta, \alpha)$ for each $\mathrm{S}$ such that $\operatorname{deg}(\mathrm{S})=0$. We will not need the dimension result in what follows and so we leave this as an exercise for the reader.

\section{The BGG-resolutions for quiver Hecke algebras}

We are now ready to prove (a stronger version of) Theorem B from the introduction over $\mathbb{k}$ an arbitrary field. Given $\alpha \in \mathcal{F}_{n}^{\ell}(h)$, we define an associated $H_{n}(\kappa)$-complex and show that this complex forms a BGG resolution of $D_{n}(\alpha)$. We simultaneously construct bases and representing matrices for $D_{n}(\alpha)$ and completely determine its restriction along the tower of cyclotomic quiver Hecke algebras.

Following a construction going back to work of Bernstein-Gelfand-Gelfand and Lepowsky $[1,28]$, we are going to define a complex of graded $A_{n}(\kappa)$-modules

$$
\cdots \longrightarrow \Delta_{2} \stackrel{\delta_{2}}{\longrightarrow} \Delta_{1} \stackrel{\delta_{1}}{\longrightarrow} \Delta_{0} \stackrel{\delta_{0}}{\longrightarrow} 0
$$

where

$$
\Delta_{\ell}:=\bigoplus_{w \in \mathcal{P}_{\ell}} \Delta(w)\langle\ell(w)\rangle
$$

We will refer to this as the BGG complex. For a diamond $(\alpha, \beta, \gamma, \delta)$ we have homomorphisms of $A_{n}(\kappa)$-modules

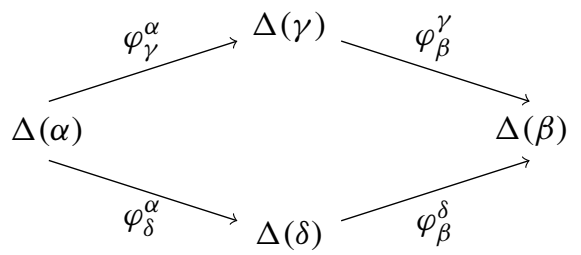

given by our one-column homomorphisms. By an easy variation on [1, Lemma 10.4] and Theorem 3.17, it is possible to assign a sign $\varepsilon(\nu, \mu)$ for each of the four homomorphisms $\varphi_{\mu}^{v}$ such that for every diamond the product of the signs associated to its four arrows is equal to $-\varepsilon_{\alpha, \beta, \gamma, \delta}$. For a strand $(\alpha, \beta, \gamma)$ we have homomorphisms 


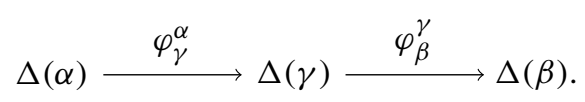

We can now define the $A_{n}(\kappa)$-differential $\delta_{\ell}: \Delta_{\ell} \rightarrow \Delta_{\ell-1}$ for $\ell \geq 1$ to be the sum of the maps

$$
\varepsilon(v, \mu) \varphi_{\mu}^{v}: \Delta(v)\langle\ell\rangle \rightarrow \Delta(\mu)\langle\ell-1\rangle
$$

over all diamond pairs $(\nu, \mu)$ with $\ell(\nu)=\ell$. For $\lambda \in \mathcal{F}_{n}^{\ell}(h)$, we set $\mathbf{C}_{\bullet}(\lambda)=$ $\bigoplus_{\ell \geq 0} \Delta_{\ell}\langle\ell\rangle$ together with the differential $\left(\delta_{\ell}\right)_{\ell \geq 0}$. We remark that each standard module occurs with a grading shift which coincides with the homological degree in which the module occurs within the complex. This grading shift/homological degree is given by the length function of the indexing $\ell$-partition.

Proposition 4.1 We have that $\operatorname{Im}\left(\boldsymbol{\delta}_{\ell+1}\right) \subseteq \operatorname{ker}\left(\boldsymbol{\delta}_{\ell}\right)$, in other words $\mathbf{C}_{\bullet}(\lambda)$ is a complex.

Proof This is a standard argument using Theorem 3.17 and the fact that if $\ell\left(w_{\alpha}\right)=$ $\ell\left(w_{\beta}\right)+2$ and $w_{\beta} \leq w_{\alpha}$, then there exists precisely two points $\gamma, \delta \in \mathbb{E}_{h, \ell}$ such that $w_{\alpha} \geq w_{\gamma}, w_{\delta} \geq w_{\beta}$.

We now apply the Schur functor to the above to obtain a complex of modules in the quiver Hecke algebra as follows,

$$
C_{\bullet}(\lambda):=\mathrm{E}_{\omega} \mathbf{C}_{\bullet}(\lambda)=\bigoplus_{\lambda \unrhd_{\mu}} S_{n}(\mu)[\ell(\mu)] \quad \text { with } \quad \mathrm{E}_{\omega} \boldsymbol{\delta}_{\ell}=\delta_{\ell} .
$$

Theorem 4.2 Let $e>h \ell$, let $\kappa \in \mathbb{Z}^{\ell}$ be $h$-admissible, let $\mathbb{k}$ be a field, and $\lambda \in \mathcal{F}_{n}^{\ell}(h)$. The $H_{n}(\kappa)$-complex $C_{\bullet}(\lambda)$ is exact except in degree zero, where

$$
H_{0}\left(C_{\bullet}(\lambda)\right)=D_{n}(\lambda)
$$

We have $D_{n}(\lambda)=\mathbb{k}\left\{c_{\mathrm{s}} \mid \mathrm{s} \in \operatorname{Std}_{e}(\lambda)\right\}$ and $\operatorname{rad}\left(S_{n}(\lambda)\right)=\mathbb{k}\left\{c_{\mathrm{s}} \mid \mathrm{s} \in \operatorname{Std}(\lambda) \backslash \operatorname{Std}_{e}(\lambda)\right\}$. Furthermore,

$$
\operatorname{res}_{n-1}^{n}\left(D_{n}(\lambda)\right)=\bigoplus_{\square \in \mathcal{F}_{h}(\lambda)} D_{n}(\lambda-\square)
$$

Proof We assume, by induction, that if $\lambda \in \mathcal{F}_{n-1}^{\ell}(h)$, then the complex $C_{\bullet}(\lambda)$ forms a BGG resolution and that $\left\{c_{\mathrm{s}} \mid \mathrm{s} \in \operatorname{Std}_{e}(\lambda)\right\}$ forms a basis of the simple module $D_{n}(\lambda)$. We now assume that $\lambda \in \mathcal{F}_{n}^{\ell}(h)$ and consider the complex $C_{\bullet}(\lambda)$. We have that

$$
\operatorname{res}_{n-1}^{n}\left(C_{\bullet}(\lambda)\right)=\bigoplus_{r \in \mathbb{Z} / e \mathbb{Z}} E_{\omega}^{r}\left(C_{\bullet}(\lambda)\right)
$$

We now consider one residue at a time. As $\lambda$ belongs to an alcove, we have that $\lambda$ (and any $\mu \triangleleft \lambda$ ) has either 0 or 1 removable $r$-boxes for each $r \in \mathbb{Z} / e \mathbb{Z}$. We let $E_{\omega}^{r}(\lambda)$ 
denote the unique $\ell$-composition (respectively $\ell$-partition) which differs from $\lambda$ by removing an $r$-node. For each residue, there are two possible cases.

- We have that $\mathrm{E}_{\omega}^{r}(\lambda)$ lies on an alcove wall or $\mathrm{E}_{\omega}^{r}(\lambda) \notin \mathscr{P}_{n}^{\ell}$. By restriction, we have that $\operatorname{Im}\left(E_{\omega}^{r} \delta_{\ell+1}\right) \subseteq \operatorname{ker}\left(E_{\omega}^{r} \delta_{\ell}\right)$ and so $E_{\omega}^{r}\left(C_{\bullet}(\lambda)\right)$ forms a complex. We have that $E_{\omega}^{r}(\lambda)$ is fixed by reflection through some hyperplane and the $\ell$-compositions of $n$ which dominate $\lambda \in \mathcal{F}_{n}^{\ell}(h)$ come in pairs $\left(v^{+}, v^{-}\right)$with $v^{-} \triangleright v^{+}$and $\ell\left(v^{+}\right)=$ $\ell\left(v^{-}\right)+1$ and furthermore such that

$$
\mathrm{E}_{\omega}^{r}\left(v^{+}\right)=\mathrm{E}_{\omega}^{r}\left(v^{-}\right)=v \in \widetilde{\mathfrak{S}}_{h \ell} \cdot\left(\mathrm{E}_{\omega}^{r}(\lambda)\right)
$$

We have that

$$
\mathrm{E}_{\omega}^{r}\left(S_{n}\left(v^{+}\right)\right)=\mathrm{E}_{\omega}^{r}\left(S_{n}\left(v^{-}\right)\right)= \begin{cases}0 & \text { if either } v^{+} \notin \mathscr{P}_{n}^{\ell} \text { or } v^{-} \notin \mathscr{P}_{n}^{\ell} \\ S_{n-1}(v) & \text { otherwise }\end{cases}
$$

Thus $\mathrm{E}_{\omega}^{r}\left(\oplus_{\lambda \unrhd_{\mu}} S_{n}(\mu)\langle\ell(\mu)\rangle\right)$ decomposes as follows,

$$
\mathrm{E}_{\omega}^{r}\left(\oplus_{\lambda \unrhd \mu} S_{n}(\mu)\langle\ell(\mu)\rangle\right)=\bigoplus_{\nu \leqslant \lambda-\square} S_{n-1}(\nu)\langle\ell(\nu)-1\rangle \bigoplus_{\nu \leqslant \lambda-\square} S_{n-1}(v)\langle\ell(\nu)\rangle
$$

Given $v \preccurlyeq \lambda-\square$, the restriction of $\phi_{v^{-}}^{v^{+}} \in \operatorname{Hom}_{H_{n}(\kappa)}\left(S_{n}\left(v^{+}\right), S_{n}\left(v^{-}\right)\right)$is equal to

$$
\operatorname{id}_{v}\langle 1\rangle \in \operatorname{Hom}_{H_{n-1}(\kappa)}\left(S_{n-1}(v)\langle\ell(v)-1\rangle, S_{n-1}(v)\langle\ell(v)\rangle\right)
$$

by equation (3.2). By restriction, we have

$$
\operatorname{Im}\left(\mathrm{E}_{\omega}^{r}\left(\delta_{\ell+1}\right)\right) \subseteq \operatorname{ker}\left(E_{\omega}^{r}\left(\delta_{\ell}\right)\right)
$$

and by equation (4.6), we have that $E_{\omega}^{r} \delta_{\ell+1}=\sum_{\ell(v)=\ell+1} 1_{v}\langle 1\rangle+\ldots$ and so the complex is exact. We conclude that $H\left(\mathrm{E}_{\omega}^{r}\left(C_{\bullet}(\lambda)\right)=0\right.$.

- We have that $\mathrm{E}_{\omega}^{r}(\lambda) \in \mathcal{F}_{n-1}^{\ell}(h)$. We have that

$$
\mathrm{E}_{\omega}^{r} S_{n}(\mu)\langle\ell(\mu)\rangle=S_{n-1}(\mu-\square)\langle\ell(\mu-\square)\rangle
$$

if $\operatorname{Rem}_{r}(\mu) \neq \emptyset$ and is zero otherwise. In the non-zero case, this is simply because $\mu-\square$ belongs to the same alcove as $\mu$ (and therefore the lengths coincide) for $\mu \leqslant \lambda$. Now, for a pair $\mu, \mu^{\prime}$ with $\square \in \operatorname{Rem}_{r}(\mu)$ and $\square^{\prime} \in \operatorname{Rem}_{r}\left(\mu^{\prime}\right)$, we have that $\mathrm{E}_{\omega}^{r} \phi_{\mu^{\prime}}^{\mu}=\phi_{\mu^{\prime}-\square}^{\mu-\square}$ by equation (3.1). Thus $\mathrm{E}_{\omega}^{r}\left(C_{\bullet}(\lambda)\right)=C_{\bullet}(\lambda-\square)$ and the right hand side is exact except $H_{0}\left(C_{\bullet}(\lambda-\square)\right)=D_{n-1}(\lambda-\square)$ by our inductive assumption. Thus $\mathrm{E}_{\omega}^{r}\left(H_{0}\left(C_{\bullet}(\lambda)\right)\right)=H_{0}\left(C_{\bullet}(\lambda-\square)\right)=D_{n-1}(\lambda-\square)$ and $E_{\omega}^{r}\left(H_{j}\left(C_{\bullet}(\lambda)\right)\right)=0$ for all $j>0$. 
Putting all of the above together, we have shown that

$$
\operatorname{res}_{n-1}^{n}\left(H_{j}\left(C_{\bullet}(\lambda)\right)\right)= \begin{cases}\bigoplus_{\square \in \mathcal{F}_{h}(\lambda)} D_{n-1}(\lambda-\square) & \text { if } j=0 \\ 0 & \text { otherwise }\end{cases}
$$

Now, since $\operatorname{Head}\left(S_{n}(\lambda)\right)=D_{n}(\lambda) \not \subset \operatorname{Im}\left(\delta_{1}\right)$, we are able to conclude that

$$
\operatorname{res}_{n-1}^{n}\left(D_{n}(\lambda)\right) \subseteq \bigoplus_{\square \in \mathcal{F}_{h}(\lambda)} D_{n}(\lambda-\square)
$$

Conversely, we have that

$$
\left|\operatorname{Std}_{e}(\lambda)\right|=\sum_{\square \in \mathcal{F}_{h}(\lambda)}\left|\operatorname{Std}_{e}(\lambda-\square)\right|
$$

by Proposition 2.11. By induction, the right hand side of Eq. (4.9) is equal to the dimension of the right hand side of equation (4.8). The lefthand-side of equation (4.9) is a lower bound for the dimension of the lefthand-side of equation (4.8). Putting these two things together, we deduce that

$$
\operatorname{res}_{n-1}^{n}\left(D_{n}(\lambda)\right)=\bigoplus_{\square \in \mathcal{F}_{h}(\lambda)} D_{n}(\lambda-\square)
$$

and furthermore, the set $\left\{c_{\mathrm{s}} \mid \mathrm{s} \in \operatorname{Std}_{e}(\lambda)\right\}$ does indeed form a basis of $D_{n}(\lambda)$; to obtain the basis of the radical, recall that $e_{\mathrm{t}} L(\mu)=0$ for $\lambda \triangleright \mu$ and $\mathrm{t} \in \operatorname{Std}_{e}(\lambda)$. Putting equation (4.7) and equation (4.10) together, we have that

$\operatorname{res}_{n-1}^{n}\left(H_{j}\left(C_{\bullet}(\lambda)\right)\right)=\left\{\begin{array}{ll}\operatorname{res}_{n-1}^{n} D_{n}(\lambda) & \text { if } j=0 \\ 0 & \text { otherwise }\end{array} H_{j}\left(C_{\bullet}(\lambda)\right)= \begin{cases}D_{n}(\lambda) & \text { if } j=0 \\ 0 & \text { otherwise }\end{cases}\right.$

where the second equality follows because $\operatorname{res}_{n-1}^{n} D_{n}(\mu) \neq 0$ for any $\lambda \unrhd \mu$ (even though $\mathrm{E}_{\omega}^{r}\left(D_{n}(\mu)\right)=0$ is possible for a given $r \in \mathbb{Z} / e \mathbb{Z}$, as seen above).

Note that the restriction rule was used as the starting point in [37], where Kleshchev obtains results concerning the dimensions of simple modules. Weirdly, our proof deduces that the homology of the complex is equal to $D_{n}(\lambda)$, that the basis $D_{n}(\lambda)$ is of the stated form, and the restriction of the simple module is of the stated form all at once!

Theorem 4.3 For $\lambda \in \mathcal{F}_{n}^{\ell}(h)$ the action of $H_{n}(\kappa)$ on $D_{n}(\lambda)=\mathbb{k}\left\{c_{\mathrm{s}} \mid \mathrm{s} \in \operatorname{Std}_{e}(\lambda)\right\}$ is as follows:

$$
y_{k}\left(c_{\mathrm{s}}\right)=0 \quad 1 \frac{i}{\omega}\left(c_{\mathrm{s}}\right)=\delta_{\underline{i}, \text {,es(s) }} \quad \psi_{r}\left(c_{\mathrm{s}}\right)= \begin{cases}c_{\mathrm{s}_{k \leftrightarrow k+1}} & \text { if }|\operatorname{res}(\mathrm{s}(r))-\operatorname{res}(\mathrm{s}(r+1))|>1 \\ 0 & \text { otherwise }\end{cases}
$$


where $\mathrm{s}_{k \leftrightarrow k+1}$ is the tableau obtained from $\mathrm{s}$ by swapping the entries $k$ and $k+1$. In particular, the subalgebra $\left\langle y_{k}, 1 \frac{i}{\omega} \mid 1 \leq k \leq r, \underline{i} \in(\mathbb{Z} / e \mathbb{Z})^{n}\right\rangle \leq H_{n}(\kappa)$ acts semisimply on $D_{n}(\lambda)$. The weight-spaces of $D_{n}(\lambda)$ are all 1-dimensional and $D_{n}(\lambda)$ is concentrated in degree zero only. Finally, the cellular bilinear form is given by $\left\langle c_{\mathrm{s}}, c_{\mathrm{t}}\right\rangle=\delta_{\mathrm{s}, \mathrm{t}}$ for $\mathrm{s}, \mathrm{t} \in \operatorname{Std}_{e}(\lambda)$.

Proof The statements not relating to the action follow from Proposition 2.11 and equation (2.4) and Theorem 4.2. The action of the idempotents is obvious. The other zero-relations all follow because the product has non-zero degree (and the module $D_{n}(\lambda)$ is concentrated in degree 0$)$. Finally, assume $|\operatorname{res}(\mathrm{s}(r))-\operatorname{res}(\mathrm{s}(r+1))|>1$. The strands terminating at $(r, 1, \ell)$ and $(r+1,1, \ell)$ on the northern edge either do or do not cross. In the former case, we can resolve the double crossing in $\psi_{r} c_{\mathrm{S}}$ without cost by our assumption on the residues and the result follows. The latter case is trivial. Finally, notice that $\mathrm{s}_{k \leftrightarrow k+1} \in \operatorname{Std}_{e}(\lambda)$ under the assumption that $|\operatorname{res}(\mathrm{s}(r))-\operatorname{res}(\mathrm{s}(r+1))|>1$.

For the sake of reference, we also note the following corollary of Theorem 4.2.

Corollary 4.4 Let $e>h \ell$, let $\kappa \in \mathbb{Z}^{\ell}$ be $h$-admissible, let $\mathbb{k}$ be a field, and $\lambda \in \mathcal{F}_{n}^{\ell}(h)$. The $\mathbf{A}_{n}(\kappa)$-complex $\mathbf{C}_{\bullet}(\lambda)$ is exact except in degree zero, where

$$
H_{0}\left(\mathbf{C}_{\bullet}(\lambda)\right)=L(\lambda)
$$

Proof Under the conditions that $e>h \ell$ and $\kappa \in \mathbb{Z}^{\ell}$ is $h$-admissible, the idempotent $\mathrm{E}_{\omega}$ does not kill any of the simple modules $\mathbf{L}(\lambda)$ for $\lambda \in \mathscr{P}_{n}^{\ell}(h)$. (See [6, Theorem 4.9] for more details.) Therefore the corresponding Serre subcategories are Morita equivalent and the result follows.

Remark 4.5 Let $p>0$. Combinatorially computing the composition series of $S_{n}(\lambda)$ for $\lambda \in \mathscr{P}_{n}^{1}(h)$ for arbitrary primes seems to be an impossible task [63]. If we assume that $p \gg h$ is suitably large then we can use Kazhdan-Lusztig theory to combinatorially calculate $\operatorname{dim}_{\mathbb{k}}\left(D_{n}(\lambda)\right)$, this requires (as a minimum) that all partitions $\mu \in \mathscr{P}_{n}^{1}(h)$ such that $\mu \triangleleft \lambda$ belong to the first $p^{2}$-alcove [50]. This is equivalent to the requirement that the $p$-weight of $\lambda$ (defined in Sect. 5) is less than $p$. For $h=3$ this combinatorics has been conjecturally extended (in terms of billiards in an alcove geometry) to the first $p^{3}$-alcove [44]. We stress that there is no restriction on the $p$-weight of $\lambda \in \mathcal{F}_{n}^{1}(h)$. Therefore understanding the composition series of unitary Specht modules is well beyond the current state of the art. Thus our two descriptions of the simple modules $D_{n}(\lambda)$ for $\lambda \in \mathcal{F}_{n}^{1}(h)$ provide the only contexts in which these modules can currently be hoped to be understood.

Example 4.6 ( $[6$, Proposition 7.6]) Let $\ell \geq 2, e=\ell+1$, and $\kappa=(0,1,2, \ldots, \ell-1) \in$ $(\mathbb{Z} / e \mathbb{Z})^{\ell}$, and $\mathbb{k}$ be arbitrary. We have that $\lambda:=((n),(n), \ldots,(n)) \in \mathcal{F}_{n \ell}^{\ell}(h)$ and that

$$
\left[S_{n \ell}((n),(n), \ldots,(n)): D_{n \ell}(v)\right]=t^{\ell(v)}+\ldots
$$

modulo terms of lower order degree. Therefore every simple module $D(v)$ for $\lambda \triangleright v \in$ $\mathscr{P}_{n \ell}^{\ell}(1)$ appears with multiplicity at least 1 . Therefore as $n \rightarrow \infty$, the number of 
composition factors of $S_{n \ell}((n),(n), \ldots,(n))$ tends to infinity and so is impossible to compute. In contrast, the module $D_{n \ell}(v)$ is 1-dimensional and easily seen to be spanned by $c_{\mathrm{t}^{\lambda}}$ for $\mathrm{t}^{\lambda}$ as in Definition 1.4.

\section{Background on the Cherednik algebras of symmetric group and the combinatorics of unitary modules}

For the remainder of the paper, we restrict our attention to the field $\mathbb{C}$ and rational Cherednik algebras of type $G(1,1, n)$. We now discuss how the combinatorial description of resolutions simplifies for (diagrammatic) Cherednik algebras of symmetric groups. In this case, we choose to emphasize the abacus presentation of partitions. We first recall this classical combinatorial approach, then flesh out the notion of homological degree introduced in [65] that is key to [65, Conjecture 4.5], and finally identify all this as the level 1 case of the alcove geometry already studied in the previous sections.

\subsection{The abacus of a partition}

Let $\lambda \in \mathscr{P}_{n}^{1}(h)$. Then $\lambda$ can be encoded by an abacus with at least $h$ beads, where each bead stands for a column of $\lambda$. This is simply a sequence of spaces and beads which records the shape of the border of $\lambda$, since knowing the border of $\lambda$ is the same as knowing $\lambda$. We form the $\mathbb{Z}$-abacus $\mathcal{A}_{\mathbb{Z}}^{h}(\lambda)$ with $h$ beads by walking along the border from the top right corner to the bottom left corner of the Young diagram of $\lambda$, writing a space every time we walk down and a bead every time we walk left.

Example 5.1 The $\mathbb{Z}$-abaci of $\left(3^{4}, 1\right),\left(3^{3}, 2,1^{2}\right) \in \mathscr{P}_{13}^{1}(3)$ with 3 beads are as follows
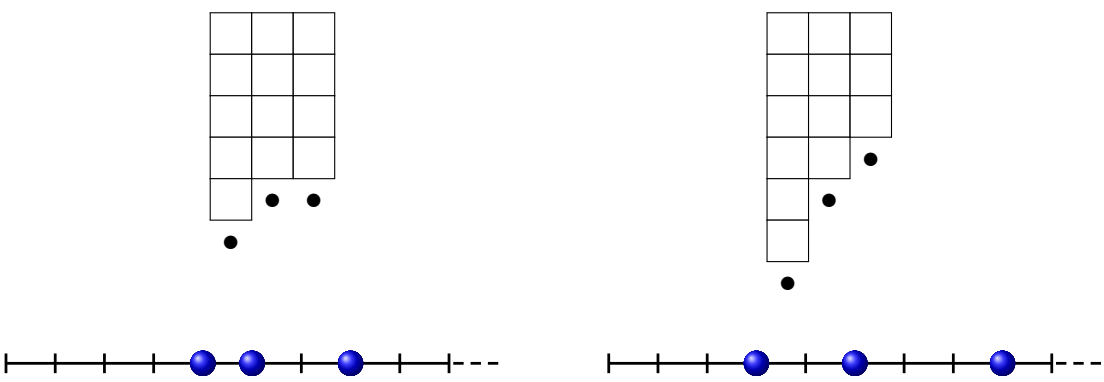

Fix $e \geq 2$. We obtain an $e$-abacus $\mathcal{A}_{e}(\lambda)$ by looping the $\mathbb{Z}$-abacus around $e$ horizontal runners. This can be described as follows: subdivide $\mathcal{A}_{\mathbb{Z}}^{h}(\lambda)$ into segments of length $e$ starting from the leftmost position, then rotate each segment counterclockwise by ninety degrees so that it is vertical. The partition is now written on $e$ horizontal runners (which we sometimes label according to residue, as in Remark 5.4). Thus the runners of our $e$-abacus resemble a musical staff, and $\mathcal{A}_{e}(\lambda)$ resembles sheet music. Like a staff, the runners of $\mathcal{A}_{e}(\lambda)$ are bounded to the left. We let them extend infinitely to the right, because we want to think of being able to move beads in that direction by adding boxes or $e$-strips at the bottom of the Young diagram of a partition. In French 
a musical score is called a partition, so we may say that our abaci are written in the French style.

Example 5.2 We picture a 5-abacus with the residues labelled.

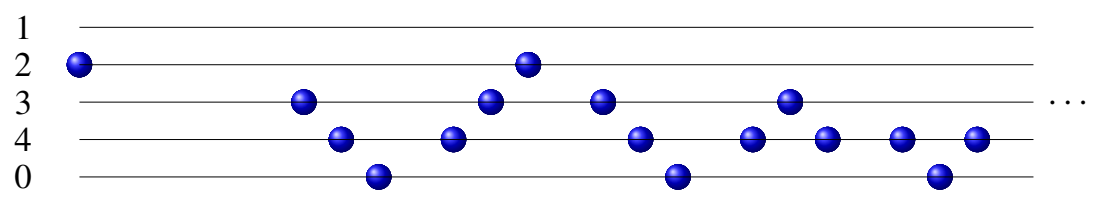

We let $w_{e}(\lambda)$ denote the total number of vacant spots which have a bead to their right and refer to this as the $e$-weight. If $w(\rho)=0$ then we say that $\rho$ is an $e$-core. Given a partition $\lambda$, we define the $e$-core of $\lambda$ to be the partition obtained by moving all beads on $\mathcal{A}_{e}(\lambda)$ as far left as possible. We let

$$
\Lambda(\rho, w):=\{\mu \vdash|\rho|+w e \mid e-\operatorname{core}(\mu)=\rho\}
$$

for $\rho$ an $e$-core.

Example 5.3 The 4 -abaci with 3 beads of $\left(3^{4}, 1\right),\left(3^{3}, 2,1^{2}\right),\left(3,2^{5}\right)$ and $\left(3^{3}, 1^{4}\right) \in$ $\mathscr{P}_{13}^{1}(3)$ are as follows
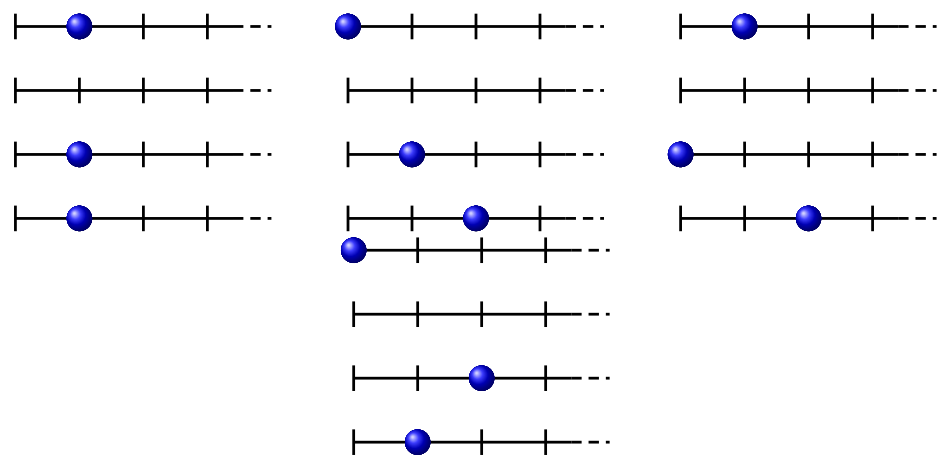

We have that $w_{4}(\lambda)=3$ and 4-core $(\lambda)=(1)$ for each of these examples.

Remark 5.4 Note that for $\mu \in \mathscr{P}_{n}^{1}(h)$, its removable box of highest content has content $h-k$, where $k$ is the position of the first bead in the $\mathbb{Z}$-abacus $\mathcal{A}_{\mathbb{Z}}^{h}(\mu)$. In particular, this bead sits in runner $k$ mod $e$ in the $e$-abacus $\mathcal{A}_{e}^{h}(\mu)$. Thus, in order to make the labels of the runners of the $e$-abaci in $\mu$ correspond in a nice way to the contents of addable and removable $i$-boxes of the partitions, one should label the runners from bottom to top by $h-1, h-2, \ldots, 1,0, e-1, e-2, \ldots, h+1, h$. With this convention, removing a box of content $i$ mod $e$ corresponds to moving a bead on runner $i-1$ down to runner $i$; and adding a box of content $i \bmod e$ corresponds to moving a bead on runner $i$ up to runner $i-1$. 


\section{2 e-unitary partitions and posets}

We recall the definition of $e$-unitary partitions from [65] and show that these are precisely the partitions in $\mathcal{F}_{n}^{1}=\cup_{h \geq 1} \mathcal{F}_{n}^{1}(h)$ studied in this paper.

Definition 5.5 [23,65] Fix $e \geq 2$. Suppose $\lambda$ has exactly $h$ columns and form $\mathcal{A}_{\mathbb{Z}}^{h}(\lambda)$ the abacus on $h$ beads. We call $\lambda$ an $e$-unitary partition if all the beads on $\mathcal{A}_{\mathbb{Z}}^{h}(\lambda)$ lie in an interval of width $e$. In particular, $\mathcal{A}_{e}(\lambda)$ has at most one bead on each runner. Given an $e$-unitary partition $\lambda$, we let $\operatorname{Po}_{e}(\lambda)$ denote the set of all the $e$-abaci obtained from $\lambda$ by successively moving a bead on some runner one step to the right so long as we also move a bead on a different runner one step to the left.

Example 5.6 When $e=4,\left(3^{4}, 1\right)$ is a 4-unitary partition, and $\left(3^{3}, 2,1^{2}\right),\left(3,2^{5}\right)$, $\left(3^{3}, 1^{4}\right) \in \mathrm{Po}_{4}(\lambda)$.

Proposition 5.7 The set $\mathcal{F}_{n}^{1}=\cup_{h \geq 1} \mathcal{F}_{n}^{1}(h)$ is precisely equal to the set of e-unitary partitions.

Proof Suppose $\lambda \in \mathcal{P}_{n}^{1}$ has exactly $h$ columns and let $\gamma_{h}, \gamma_{1}$ denote the positions of the leftmost and rightmost beads on $\mathcal{A}_{\mathbb{Z}}^{h}(\lambda)$. Now simply note that $\gamma_{1}-\gamma_{h} \leq e-1$ if and only if $\left\langle\lambda+\rho, \varepsilon_{1}-\varepsilon_{h}\right\rangle<e-1$ if and only if $\lambda \in \mathcal{F}_{n}^{1}(h)$.

Remark 5.8 If $e=h$ then $\lambda$ is $e$-unitary if and only if $\lambda=\left(e^{k}\right)$ for some $k \in \mathbb{N}$. If $\lambda$ is an $e$-unitary partition, then any $\mu \in \operatorname{Po}_{e}(\lambda)$ is always $e$-restricted unless $\lambda=\left(e^{k}\right)$ and $\mu=\lambda$.

If an $e$-abacus $\mathcal{A}_{e}(\mu)$ has at most one bead on each runner, let $b_{i}$ be the unique bead on the runner labeled $i$ if such a bead exists, and let $\beta_{i} \in \mathbb{Z}_{\geq 0}$ be the horizontal position of $b_{i}$. Sometimes by abuse of notation we might just refer to $\beta_{i}$ as a bead. We say that we shift the bead $b_{i}$ one unit to the left if we replace $\beta_{i}$ with $\beta_{i}-1$. The effect of this on the Young diagram of $\mu$ is to remove what is called an $e$-rimhook from the border of $\mu$ : a connected subset $R$ of $e$ boxes of the Young diagram of $\mu$ such that if $b \in R$ is in row $r$ and column $c$, then there is no box in the Young diagram of $\mu$ situated in row $r+1$ and column $c+1$. Similarly, shifting $b_{i}$ one unit to the right adds an $e$-rimhook to $\mu$. That means precisely that shifting $b_{i}$ one unit to the right adds $e$ boxes $x_{1}, \ldots, x_{e}$ to $\mu$ in such a way that $x_{1}, \ldots, x_{e}$ form an $e$-rimhook in the border of the partition $\mu \cup\left\{x_{1}, \ldots, x_{e}\right\}$, removing which yields back $\mu$.

Example 5.9 Let $h=3$ and $e=4$. We continue with the example of $\lambda=(3,3,3,1)$. Shifting the bead on the top runner one unit to the left removes the green 4-rimhook from $\lambda$ yielding $(3,3,3)$ as pictured below. Shifting the bead on the bottom runner one unit to the right then adds a 4-rimhook to $(3,3,3)$ yielding $(3,3,3,2,1,1)$.
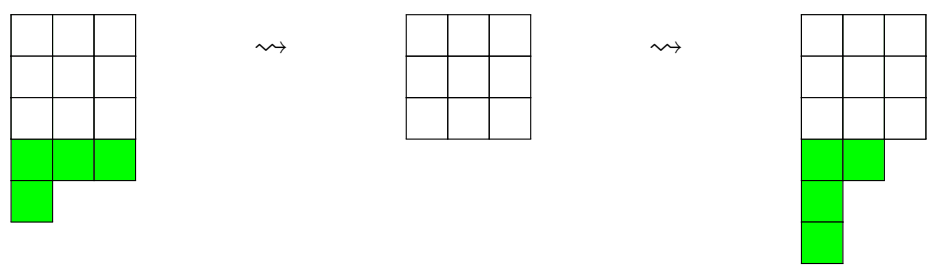

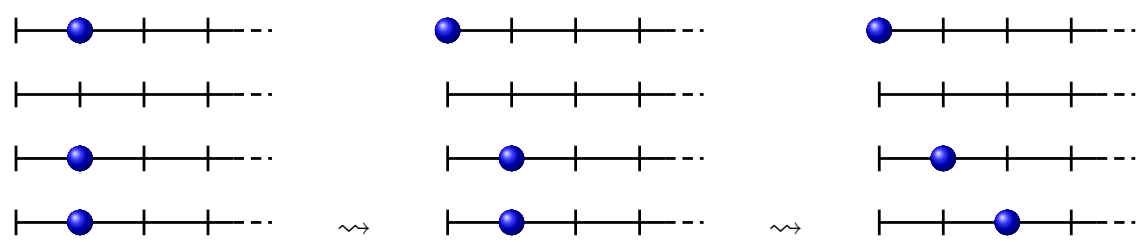

\subsection{The affine and extended affine symmetric group actions}

There is a natural action of the affine symmetric group $\widetilde{\mathfrak{S}}_{h}$ on $\mathrm{Po}_{e}(\lambda)$ when we take the presentation of $\widetilde{\mathfrak{S}}_{h}$ given by generators $s_{i}, i \in \mathbb{Z} / h \mathbb{Z}$, subject to the relations $s_{i}^{2}=1$, $s_{i} s_{j}=s_{j} s_{i}$ if $|i-j|>1$, and $s_{i} s_{i+1} s_{i}=s_{i+1} s_{i} s_{i+1}$ (where all subscripts are taken $\bmod h) . \mathfrak{S}_{h}=\left\langle s_{1}, \ldots, s_{h-1}\right\rangle$ acts by permutation of the $h$ runners containing beads, while $s_{0}$ switches the top and bottom beads in the abacus, then moves the bottom bead one step to the right and the top bead one step to the left. From the description of $\mathrm{Po}_{e}(\lambda)$ in Definition $5.5, \widetilde{\mathfrak{S}}_{h}$ acts transitively on $\operatorname{Po}_{e}(\lambda)$.

Example 5.10 Illustration of the action of $s_{0}$ :
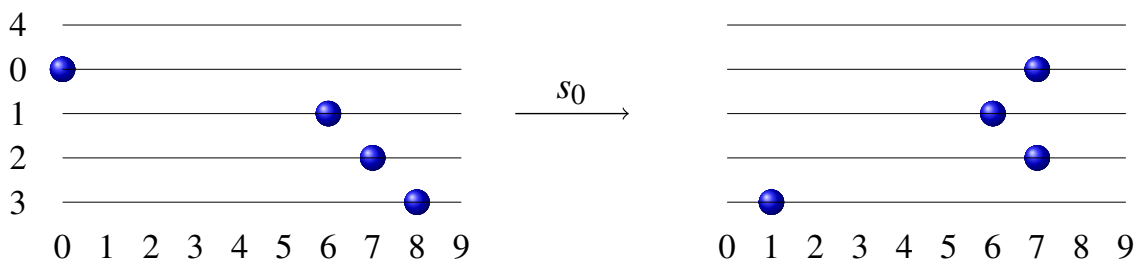

The extended affine symmetric group $\widehat{\mathfrak{S}}_{h}$ is the semidirect product $\mathbb{Z}^{h} \rtimes \mathfrak{S}_{h}$. There is a natural action of $\widehat{\mathfrak{S}}_{h}$ on the set of $e$-abaci with exactly one bead on a fixed subset of $h$ runners, and no beads on the other runners: $\mathbb{Z}^{h}$ acts as the group of horizontal translations of the beads on their runners, and $\mathfrak{S}_{h}$ as permutations of the $h$ runners containing the beads. This action is locally nilpotent for the subgroup $\mathbb{Z}_{<0}^{h}$ consisting of left translations of the beads. In terms of partitions, the meaning is as follows: let $\rho$ be an $e$-core of some unitary partition; equivalently, $\mathcal{A}_{e}(\rho)$ has its beads pushed all the way to the left and they are concentrated in the leftmost column of the $e$-abacus. Let $\mathcal{P}_{e}(\rho)_{h}$ be the union of all $\operatorname{Po}_{e}(\lambda), \lambda$ an $e$-unitary partition such that the $e$-core of $\lambda$ is $\rho$ and $\lambda$ has $h$ columns. Let $\epsilon_{i}=(0, \ldots, 1, \ldots, 0) \in \mathbb{Z}^{h}$ with the 1 in the $i$ 'th position. Then $\epsilon_{i}$ acts on $\mu \in \mathcal{P}_{e}(\rho)_{h}$ by shifting the bead on the $i$ 'th runner containing a bead one unit to the right; on the Young diagram of $\mu$ it adds an $e$-rimhook whose arm-length is at most $h-1$. Observe that $\mathcal{P}_{e}(\rho)_{h}$ is generated by $\mathcal{A}_{e}(\rho)$ under the action of $\widehat{\mathfrak{S}}_{h}$ :

$$
\widehat{\mathfrak{S}}_{h} \cdot \mathcal{A}_{e}(\rho)=\mathcal{P}_{e}(\rho)_{h}
$$

$\mathcal{P}_{e}(\rho)_{h}$ is naturally identified with the monoid $\mathbb{Z}_{\geq 0}^{h}$ as a left $\widehat{\mathfrak{S}}_{h}$-module by identifying an abacus $\mathcal{A} \in \mathcal{P}_{e}(\rho)_{h}$ with the $h$-tuple of its beads' positions $\left(\beta_{1}, \ldots, \beta_{h}\right) \in \mathbb{Z}_{\geq 0}^{h}$. Note that adding or removing empty runners from the $e$-abacus (thus changing $e$ while 
keeping $h$ fixed) does not affect the action of $\widehat{\mathfrak{S}}_{h}$ which acts only on the non-empty runners.

\subsection{The homological degree statistic}

Let $\lambda$ be an $e$-unitary partition. We recall the homological degree statistic on $\operatorname{Po}_{e}(\lambda)$ introduced by Berkesch-Griffeth-Sam.

Definition 5.11 Suppose $\mathcal{A}$ is an $e$-abacus with at most one bead on each runner. A disorder of $\mathcal{A}$ is an unordered pair $\{i, j\}$ such that runners $i$ and $j$ both contain a bead, satisfying $\beta_{i}>\beta_{j}$ and $b_{j}$ is above $b_{i}$. In other words, a pair of beads of $\mathcal{A}$ yields a disorder if one bead is above and strictly to the left of the other bead.

Definition 5.12 [65, Definition 4.3] Let $\mu \in \operatorname{Po}_{e}(\lambda)$. The homological degree of $\mu$, written $\mathrm{hd}(\mu)$, is the sum of the differences of all horizontal positions of beads in $\mathcal{A}_{e}(\mu)$ minus the number of disorders of $\mathcal{A}_{e}(\mu)$ :

$$
\operatorname{hd}(\mu)=\sum_{\substack{i, j \in \mathbb{Z} / e Z \\ b_{i}, b_{j} \neq \varnothing \\ b_{j} \text { is below } b_{i}}}\left|\beta_{i}-\beta_{j}\right|-\#\left\{\text { disorders of } \mathcal{A}_{e}(\mu)\right\}
$$

Example 5.13 In Example 5.10, let $v$ denote the partition whose abacus is on the left, and let $\mu=s_{0}(v)$ as in the picture. Then $\mathcal{A}_{5}(v)$ has 6 disorders and hd(v) $=1+2+$ $8+1+7+6-6=19 ; \mathcal{A}_{5}(\mu)$ has 1 disorder and hd $(\mu)=6+5+6+1+1-1=18$. Observe that $s_{0}$ changed the homological degree by 1 .

\subsection{Homological degree produced recursively by elements of $\widehat{\mathfrak{S}}_{e}$}

Notice that empty runners of $\mathcal{A}_{e}(\mu)$ play no role in $\mathrm{hd}(\mu)$; if the empty runners are removed from $\mathcal{A}_{e}(\mu)$, the homological degree remains the same. For simplicity of the formulas and exposition, we therefore work in the case that there are no empty runners, that is, $h=e$ columns and $\lambda=\left(e^{k}\right)$ for some $k \in \mathbb{N}$. The empty runners can be put back in at the end.

Our first characterization of the homological degree produces this statistic recursively starting from the empty partition by applying sequences of special elements $\tau_{i} \in \widehat{\mathfrak{S}}_{e}, i=e-1, e-2, \ldots, 1,0$, in a non-increasing order with respect to $i$.

Definition 5.14 Let $\tau_{i} \in \widehat{\mathfrak{S}}_{e}$ be defined as follows: $\tau_{i}$ fixes the bottom $i$ runners; on the top $e-i$ runners, it first cyclically rotates the beads in the topwards direction, then shifts one space to the right the bead on the $(e-i)$ 'th runner from the top.

Each $\tau_{i}$ is the "affine generator" of the subgroup $\widehat{\mathfrak{S}}_{e-i}$ of $\widehat{\mathfrak{S}}_{e}$ which fixes the bottom $i$ runners: $\tau_{i}$ together with $\mathfrak{S}_{e-i}$ generates $\widehat{\mathfrak{S}}_{e-i}$ [41, Section 2.1]. We are interested in applying $\tau_{i}$ to abaci whose bottom $i$ runners have their beads pushed all the way to the left. 
Example 5.15 Consider the 5-abacus of $\left(3^{11}, 2^{3}, 1^{11}\right)$. Then $\tau_{2}$ acts as follows:
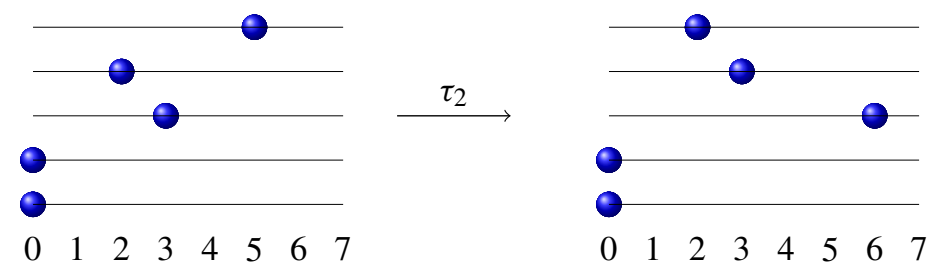

Observe that $\tau_{2}$ increased the homological degree of the abacus by 2 .

Suppose $\tau$ is a partition all of whose parts are of size at most $e-1$, and which may contain parts of size 0 , so $\tau=\left((e-1)^{a_{e-1}},(e-2)^{a_{e-2}}, \ldots, 1^{a_{1}}, 0^{a_{0}}\right)$. Thus $\tau$ fits inside an $e-1$ by $k$ box, where $k$ is the total number of parts of $\tau$. Now identify $\tau$ with the element of $\widehat{\mathfrak{S}}_{e}$ given by the composition of operators $\tau_{0}^{a_{0}} \tau_{1}^{a_{1}} \ldots \tau_{e-1}^{a_{e-1}}$. By abuse of notation we will also call this element $\tau$. The proof of the following lemma is straightforward:

Lemma 5.16 Let $\tau=\left((e-1)^{a_{e-1}},(e-2)^{a_{e-2}}, \ldots, 1^{a_{1}}, 0^{a_{0}}\right)$ with $\sum_{i=0}^{e-1} a_{i}=k \in \mathbb{Z}_{\geq 0}$. Then $\tau\left(\mathcal{A}_{e}(\emptyset)\right)=\mathcal{A}_{e}(\mu)$ with $\mu \in \operatorname{Po}_{e}\left(e^{k}\right)$. Any $\mu \in \operatorname{Po}_{e}\left(e^{k}\right)$ is produced in this way from a unique such $\tau$, and we have:

$$
\operatorname{hd}(\mu)=\sum_{i=0}^{e-1} i a_{i}=|\tau|
$$

Let $\lambda$ be an arbitrary $e$-unitary partition. By removing the empty runners from the $e$-abaci in $\operatorname{Po}_{e}(\lambda)$, there is likewise a natural bijection between the partitions $\mu$ in $\mathrm{Po}_{e}(\lambda)$ and partitions $\tau$ which fit inside an $(h-1)$ by $k$ box,

$$
\left\{\mu \in \operatorname{Po}_{e}(\lambda)\right\} \stackrel{\Phi}{\stackrel{ }{\leftrightarrows}}\left\{\tau \subset(h-1)^{k}\right\},
$$

given by $\Phi(\tau)=\tau(\emptyset)$ (where $\tau$ on the right-hand-side is the corresponding element of $\widehat{\mathfrak{S}}_{h}$ as described above). This bijection identifies $h d(\mu)$ with $|\tau|$.

Remark 5.17 Such a bijection turns up elsewhere in representation theory: notably, partitions $\tau$ which fit inside an $(h-1)$ by $k$ box also parametrize (1) the simple and standard modules of a regular block $\mathcal{B}^{\mathfrak{p}}$ of parabolic category $\mathcal{O}^{\mathfrak{p}}$ for $\mathfrak{g l}(h-1+k)$ with respect to the maximal parabolic $\mathfrak{g l}(h-1) \times \mathfrak{g l}(k)$ [55]; (2) the Schubert cells in the Grassmannian $\operatorname{Gr}(k, h-1+k)=\operatorname{Gr}(h-1, h-1+k)$. The category $\mathcal{B}^{\mathfrak{p}}$ is equivalent to perverse sheaves on the Grassmannian [9,55], explaining the coincidence of (1) and (2). Let $L_{(h-1)^{k}}$ denote the simple module in $\mathcal{B}^{\mathfrak{p}}$ labeled by $\tau=(h-1)^{k}$, the unique maximal element of the poset (the poset structure is given by inclusion of Young diagrams). The bijection following Lemma 5.16 identifies the labels of the characters of unitary $L(\lambda) \in\left[\mathcal{O}_{1 / e}\left(\mathfrak{S}_{n}\right)\right]$ and $L_{(h-1)^{k}} \in\left[\mathcal{O}^{\mathfrak{p}}\right]$. Moreover, $L_{(h-1)^{k}}$ has a BGG resolution [4] and via the bijection $\Phi$ we obtain a natural bijection between the Verma modules appearing in degree $i$ of the respective resolutions in $\mathcal{O}_{1 / e}\left(S_{n}\right)$ and 
$\mathcal{O}^{\mathfrak{p}}$. However, the categories $\mathcal{O}_{1 / e}\left(\mathfrak{S}_{n}\right)_{\leq \lambda}$ and $\mathcal{B}^{\mathfrak{p}}$ are not equivalent if $k>2$, and as a poset $\operatorname{Po}_{e}(\lambda)$ has "extra edges" coming from the $\widetilde{\mathfrak{S}}_{h}$-action if $k>2$.

\subsection{Homological degree via rimhooks of minimal leg-length}

Consider again the case that there is exactly one bead on every runner of the abacus. By the definition of $\tau_{i}$, it follows that the effect of applying $\tau=\left((e-1)^{a_{e-1}},(e-\right.$ $\left.2)^{a_{e-2}}, \ldots, 1^{a_{1}}, 0^{a_{0}}\right)$ to the empty partition is to build a Young diagram $\lambda$ by successively dropping $e$-rimhooks which meet the leftmost column (with leg-lengths $e-1$ ( $a_{e-1}$ times), $e-2$ ( $a_{e-2}$ times) and so on) Tetris-style on top of the partition constructed so far, then letting the boxes slide down the columns so that the result is a partition. This can change the shape of the previous rimhooks that were added, but not the set of their leg-lengths. Thus we obtain a second combinatorial explanation of the homological degree: if $e=h$ then $\operatorname{hd}(\lambda)$ is the sum of the leg-lengths of the $e$-rimhooks of minimal leg-length composing $\lambda$. If $e>h$ then $\mathrm{hd}(\lambda)$ is the sum of the leg-lengths of the e-rimhooks of minimal leg-length composing $\lambda$ minus $(e-h) k$, where $k=e$-weight $(\lambda)$. This can be restated in a uniform way by considering the arm-lengths instead of the leg-lengths of the rimhooks: $\operatorname{hd}(\mu)$ is equal to $(h-1) k$ minus the sum of armlengths of the (minimal leg-length) rimhooks removed.

Example 5.18 Let $e=h=5$ and $\tau=(3,3,1,0)$. Then $\tau(\emptyset)=\left(5,4,2^{5}, 1\right)=: \lambda$ and $\operatorname{hd}(\lambda)=7$. We show the process of applying $\tau$ on abaci and partitions and the four 5-rimhooks of minimal leg-lengths $3,3,1,0$ which compose $\lambda$ :

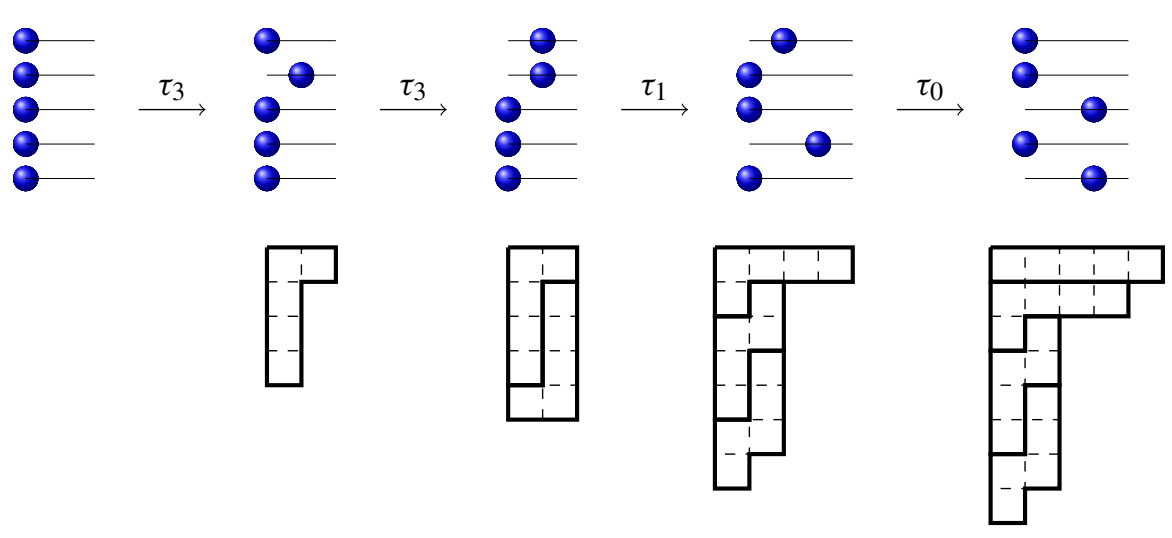

\subsection{Homological degree is the length function.}

We now give a third combinatorial description of the homological degree by identifying it with the length function on $\widetilde{\mathfrak{S}}_{h}$. This unifies the combinatorics of abaci with that of alcove geometries and allows us to describe the BGG complex in type $A$ in terms of abaci. Let $\lambda$ be a unitary partition, and suppose $\lambda$ has $h<e$ columns. 
(1)

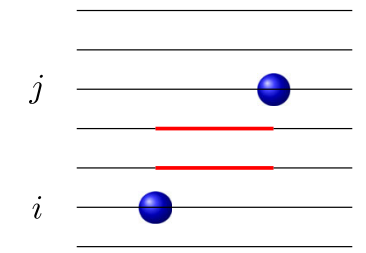

$(2)$

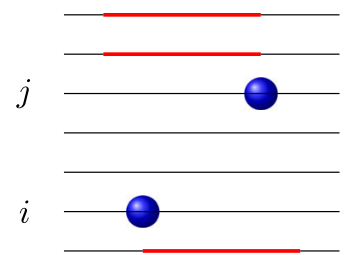

Fig. 4 The conditions (1) and (2) on abaci in Lemma 5.19 in a picture: applying $t$ will increase the homological degree by 1 if and only if no bead lies in the red regions of the runners

Lemma 5.19 The following are equivalent for $v, \mu \in \operatorname{Po}_{e}(\lambda), \mu \triangleright v$ :

- $\ell(v)=\ell(\mu)+1$ and $v$ is obtained from $\mu$ by moving a column of boxes as in Theorem 3.3;

- $t \mu=v$ for some transposition $t \in \widetilde{\mathfrak{S}}_{h}$ acting on abaci as above, subject to the following conditions on the beads $\beta_{i}$ of $\mathcal{A}_{e}(\mu)$ :

(1) ift $\in \mathfrak{S}_{h}$ and swaps runners $i$ and $j$, then for each runner $k$ between runners $i$ and $j, \beta_{k} \notin\left[\beta_{i}, \beta_{j}\right]$;

(2) if $t$ is conjugate to $s_{0}$ and acts nontrivially on runners $i$ and $j$, runner $i$ below runner $j$, then: for each runner $k$ below runner $i, \beta_{k} \notin\left[\beta_{i}, \beta_{j}+1\right]$ and for each runner $\ell$ above runner $j, \beta_{\ell} \notin\left[\beta_{i-1}, \beta_{j}\right]$.

Therefore, the homological degree statistic on $\mathrm{Po}_{e}(\lambda)$ coincides with the length function on $\mathrm{Po}_{e}(\lambda)$ coming from the $\widetilde{\mathfrak{S}}_{h}$ alcove geometry.

Proof This is a translation of 1-column moves from the language of Young diagrams into the language of abaci. A direct computation using Definition 5.12 shows that the conditions for a transposition $t$ to increase the homological degree by 1 are exactly those given by (1) and (2) (Fig. 4).

See Fig. 5 for an example of the results of Section 5.5. and this section.

\subsection{The Cherednik algebra of the symmetric group}

Let $\mathfrak{S}_{n}$ be the symmetric group on $n$ elements. The group $\mathfrak{S}_{n}$ acts on the algebra of polynomials in $2 n$ non-commuting variables $\mathbb{C}\left\langle x_{1}, \ldots, x_{n}, y_{1}, \ldots y_{n}\right\rangle$. Fix a number $c \in \mathbb{C}$. The rational Cherednik algebra $H_{c}\left(\mathfrak{S}_{n}\right)$ is the quotient of the semidirect product algebra $\mathbb{C}\left\langle x_{1}, \ldots, x_{n}, y_{1}, \ldots, y_{n}\right\rangle \rtimes \mathfrak{S}_{n}$ by the relations

$$
\left[x_{i}, x_{j}\right]=0, \quad\left[y_{i}, y_{j}\right]=0, \quad\left[y_{i}, x_{j}\right]=c(i j)(i \neq j), \quad\left[y_{i}, x_{i}\right]=1-c \sum_{j \neq i}(i j)
$$

where $(i j)$ denotes the transposition in $\mathfrak{S}_{n}$ that switches $i$ and $j$, see [21]. $H_{c}$ has three distinguished subalgebras: $\mathbb{C}[y]:=\mathbb{C}\left[y_{1}, \ldots, y_{n}\right], \mathbb{C}[\underline{x}]:=\mathbb{C}\left[x_{1}, \ldots, x_{n}\right]$, and the group algebra $\mathbb{C S}_{n}$. The PBW theorem [21, Theorem 1.3] asserts that multiplication gives a vector space isomorphism

$$
\mathbb{C}[\underline{x}] \otimes \mathbb{C S}_{n} \otimes \mathbb{C}[\underline{y}] \stackrel{\cong}{\rightarrow} H_{c}
$$



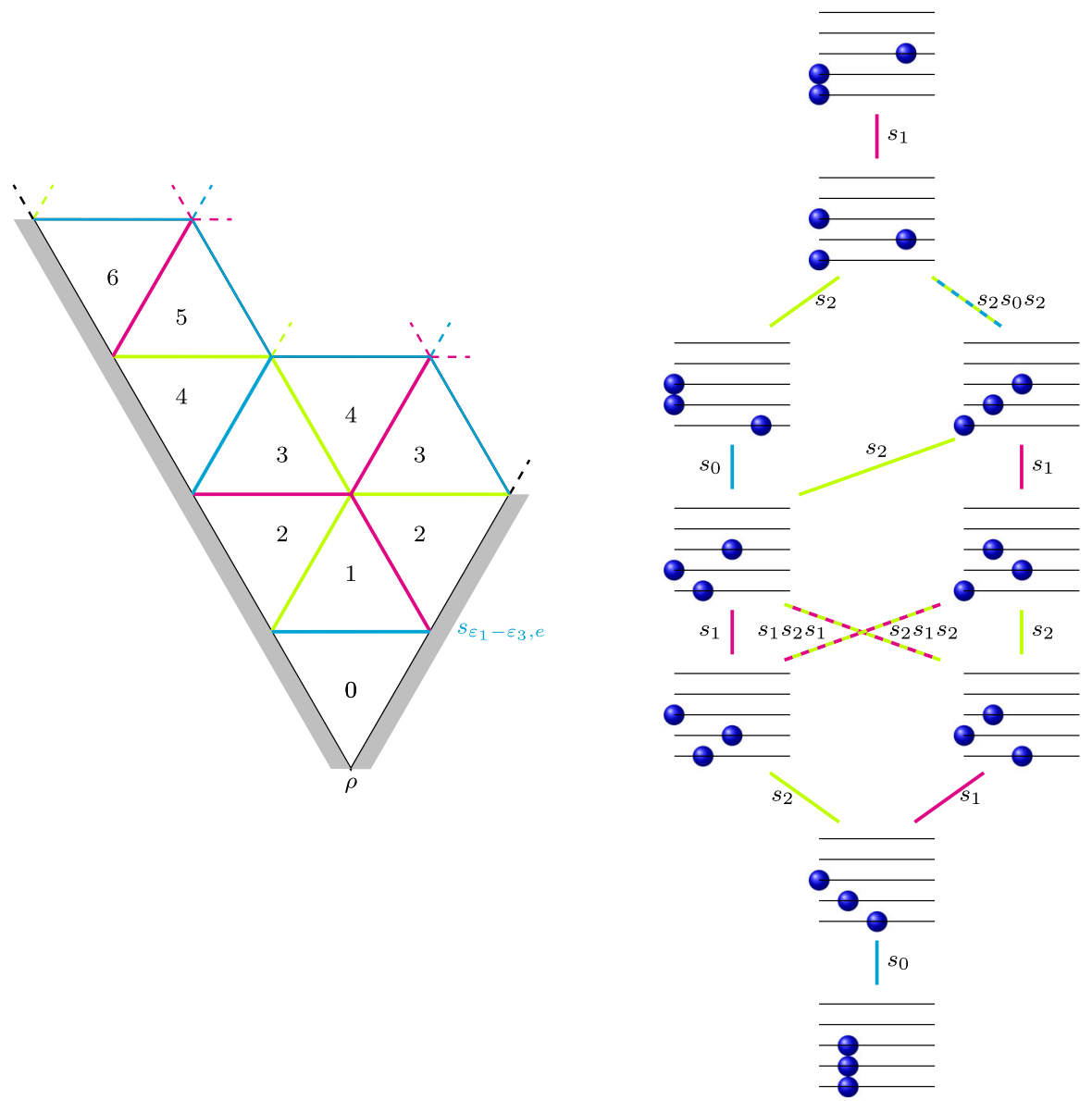

Fig. 5 On the left we have the alcoves corresponding to partitions in $\operatorname{Po}_{e}(\lambda)$ when $h=3$ and $e=5$. The fundamental alcove is at the bottom and contains $\left(3^{5}\right) \in \mathcal{F}_{15}(3)$. Each alcove contains a number indicating the length/homological degree for a point in that alcove. The grey region denotes the non-dominant region. The dotted lines indicate that we tile one sixth of $\mathbb{R}^{2}$ when we let $n \rightarrow \infty$. Crossing a wall of color $i$ corresponds to applying $s_{i}$ to the partition in that alcove, with $i: 0,1,2$. On the right-hand side, we have extracted the poset $\operatorname{Po}_{e}(\lambda)$. The homological degree increases from the bottom (where it is zero) to the top (where it is 6). The edges of the poset are coloured and decorated so as to facilitate comparison between the two pictures (colour figure online)

called the triangular decomposition of $H_{c}$, by analogy with the triangular decomposition of the universal enveloping algebra of a semisimple Lie algebra.

We define the category $\mathcal{O}_{c}\left(\mathfrak{S}_{n}\right)$ to be the full subcategory consisting of all finitely generated $H_{c}$-modules on which $y_{1}, \ldots, y_{n}$ act locally nilpotently. Category $\mathcal{O}_{c}$ is not always very interesting. By [18], see also [3, Section 3.9], $\mathcal{O}_{c}$ is semisimple (and equivalent to the category of representations of $\left.\mathfrak{S}_{n}\right)$ unless $c=r / e$, with $\operatorname{gcd}(r ; e)=1$ and $1<e \leq n$. Equivalences of categories reduce the study of $\mathcal{O}_{r / e}\left(\mathfrak{S}_{n}\right)$ to $\mathcal{O}_{1 / e}\left(\mathfrak{S}_{n}\right)$, for $1<e \leq n$ [51]. For the rest of the paper we work with $\mathcal{O}_{1 / e}\left(\mathfrak{S}_{n}\right)$, unless otherwise explicitly stated. It will be convenient to set 


$$
\mathcal{O}_{1 / e}:=\bigoplus_{n \geq 0} \mathcal{O}_{1 / e}\left(\mathfrak{S}_{n}\right)
$$

The category $\mathcal{O}_{1 / e}\left(\mathfrak{S}_{n}\right)$ is a highest weight category with respect to the ordering $\triangleright$ on $\mathscr{P}_{n}^{1}$. The standard modules are constructed as follows. For each partition $\lambda$ of $n$, let $S_{n}(\lambda)$ be the corresponding Specht module of $\mathbb{C S}_{n}$, this is an irreducible representation. Extend the action of $\mathfrak{S}_{n}$ on $S_{n}(\lambda)$ to an action of $\mathbb{C}[y] \rtimes \mathfrak{S}_{n}$ by letting $y_{1}, \ldots, y_{n}$ act by 0 . The algebra $\mathbb{C}[\underline{y}] \rtimes \mathfrak{S}_{n}$ is a subalgebra of $H_{1 / e}$ and we define

$$
\Delta_{H_{1 / e}}(\lambda):=\operatorname{Ind}_{\mathbb{C}[\underline{y}] \rtimes \mathfrak{S}_{n}}^{H_{1 / e}} S_{n}(\lambda):=H_{1 / e} \otimes \mathbb{C}[\underline{y}] \rtimes \mathfrak{S}_{n} S_{n}(\lambda)=\mathbb{C}[\underline{x}] \otimes S_{n}(\lambda)
$$

where the last equality is only as $\mathbb{C}[\underline{x}]$-modules and follows from the triangular decomposition. We let $L_{H_{1 / e}}(\lambda)$ denote the unique irreducible quotient of $\Delta_{H_{1 / e}}(\lambda)$. We often drop the subscripts on these modules when the context is clear.

Any module $M \in \mathcal{O}_{1 / e}\left(\mathbb{S}_{n}\right)$ is finitely generated over the algebra $\mathbb{C}[\underline{x}]$ and, as such, it has a well-defined support $\operatorname{supp}(M) \subseteq \mathbb{C}^{n}=\operatorname{Spec}(\mathbb{C}[\underline{x}])$. We now explain a way to compute the supports of simple modules in $\mathcal{O}_{1 / e}\left(\mathfrak{S}_{n}\right)$ that was obtained in [62]. To do this, for any $i=0, \ldots,\lfloor n / e\rfloor$, denote by $X_{i}$ the variety

$$
\begin{aligned}
X_{i}:= & \mathfrak{S}_{n}\left\{\left(z_{1}, \ldots, z_{n}\right) \in \mathbb{C}^{n}: z_{1}=z_{2}=\cdots z_{e}, z_{e+1}\right. \\
& \left.=\cdots=z_{2 e}, \cdots, z_{(i-1) e+1}=\cdots=z_{i e}\right\}
\end{aligned}
$$

By its definition, $X_{i}$ is a $\mathfrak{S}_{n}$-stable subvariety of $\mathbb{C}^{n}$. Note that $X_{0}=\mathbb{C}^{n}$, and these subvarieties form a chain $X_{0} \supsetneq X_{1} \supsetneq \cdots \supsetneq X_{\lfloor n / e\rfloor}$. Now recall that a partition $\lambda$ is said to be $e$-restricted if $\lambda_{i}-\lambda_{i+1}<e$ for every $i \geq 0$, that is, if no two consecutive parts of $\lambda$ differ by more than $e-1$ parts. By the division algorithm, for any partition $\lambda$ there exist unique partitions $\mu, v$ such that $\lambda=e \mu+v$ and $v$ is $e$-restricted. Then, according to [62, Theorem 1.6],

$$
\operatorname{supp}\left(L_{1 / e}(\lambda)\right)=X_{|\mu|}
$$

So, for example, $L_{1 / e}(\lambda)$ has full support if and only if $\lambda$ is $e$-restricted. On the other hand, if $e$ divides $n$, then $L_{1 / e}(\lambda)$ has minimal support if and only if $\lambda=e \mu$, where $\mu$ is a partition of $n / e$.

The categories $\mathcal{O}_{1 / e}\left(\mathfrak{S}_{n}\right)$ come equipped with induction and restriction functors

$$
\operatorname{Res}_{n-1}^{n}: \mathcal{O}_{1 / e}\left(\mathfrak{S}_{n}\right) \rightleftarrows \mathcal{O}_{1 / e}\left(\mathfrak{S}_{n-1}\right): \operatorname{Ind}_{n-1}^{n}
$$

that were constructed by Bezrukavnikov and Etingof in [3]. Their definition is quite technical and will not be needed. In fact, Bezrukavnikov and Etingof constructed restriction functors for any parabolic subgroup of $\mathfrak{S}_{n}$, [3]. It follows from their construction that $M$ has full support if and only if it is not killed by restriction to any parabolic subgroup. We will use this property below without further mention.

Finally, we mention the Knizhnik-Zamolodchikov functor KZ: $\mathcal{O}_{c}\left(\mathfrak{S}_{n}\right) \rightarrow$ $\mathcal{H}_{q}\left(\mathfrak{S}_{n}\right)$-mod where $q=\exp (2 \pi \sqrt{-1} c)$ and $\mathcal{H}_{q}\left(\mathfrak{S}_{n}\right)$ is the finite Hecke algebra, 
defined over $\mathbb{C}$, of the symmetric group $S_{n}$. This is an exact functor that identifies the category of $\mathcal{H}_{q}\left(\mathfrak{S}_{n}\right)$-modules with the quotient of $\mathcal{O}_{c}\left(\mathfrak{S}_{n}\right)$ by the Serre subcategory generated by those simple modules $L(\lambda)$ with proper support, see [29]. The KZ functor is fully faithful on projective objects and, when $c \notin 1 / 2+\mathbb{Z}$, it is also fully faithful on standard objects.

\subsection{Connection to diagrammatic algebra}

Crucial to our arguments is the fact that the highest weight category $\mathcal{O}$ for the Cherednik algebra of $\mathfrak{S}_{n}$ is Morita equivalent to one of the finite-dimensional diagrammatic algebra from Subsection 1.2. Let $e \geq 2$, so that we have the highest weight category $\mathcal{O}_{1 / e}\left(\mathfrak{S}_{n}\right)$. Recall from Subsection 1.2 that we can form the algebra $\mathbf{A}_{n}(\kappa)$ for $\kappa=$ $0 \in \mathbb{Z}$ and $e \geq 2$ which is a quasi-hereditary cellular algebra. We remark that the choice of the integer $\kappa \in \mathbb{Z}$ is immaterial in level 1 , however we have chosen $\kappa=0$ in order to match-up with the classical residue combinatorics we have been using thus far.

The following theorem is originally due, in a slightly different form, to Rouquier, [51]. In the version we need it, it is due to Webster [59] where the proof is a uniqueness of faithful quasi-hereditary covers argument. A constructive proof is given by Webster in [60, Theorem 3.15].

Theorem 5.20 The categories $\mathcal{O}_{1 / e}\left(\mathfrak{S}_{n}\right)$ and $\mathbf{A}_{n}(0)$-mod are highest-weight equivalent. More precisely, for every $n \geq 0$ there exist an equivalence $\Psi_{n}: \mathcal{O}_{1 / e}\left(\mathfrak{S}_{n}\right) \rightarrow$ $\mathbf{A}_{n}(0)$-mod satisfying the following properties.

(a) For every partition $\lambda \vdash n, \Psi_{n}\left(\Delta_{H_{1 / e}}(\lambda)\right)=\Delta_{\mathbf{A}_{n}(0)}(\lambda)$ and $\Psi_{n}\left(L_{H_{1 / e}}(\lambda)\right)=$ $\mathbf{L}_{\mathbf{A}_{n}(0)}(\lambda)$.

(b) The equivalences $\Psi_{n}$ intertwine the restriction functors.

(c) Up to applying the Brundan-Kleshchev isomorphism $\mathcal{H}_{q}\left(\mathfrak{S}_{n}\right) \cong H_{n}(0)$, see [10], the functor $\Psi_{n}$ intertwines the $\mathrm{KZ}$ functor and the Schur functor $\mathrm{E}_{\omega}$.

Remark 5.21 Webster's theorem [59, Theorem 4.8] (see also [60, Theorem 3.15]) is much more general and connects the algebra $\mathbf{A}_{n}(\kappa)$ for $\ell>1$ with the category $\mathcal{O}$ for the rational Cherednik algebra of the wreath product group $S_{n} \imath \mathbb{Z} / \ell \mathbb{Z}$. We will not need this more general version of the theorem.

Thanks to Theorem 5.20 we can apply our results on the algebra $\mathbf{A}_{n}(0)$ to category $\mathcal{O}_{1 / e}\left(\mathfrak{S}_{n}\right)$. We will exploit this in the next section to prove almost all cases of the Berkesch-Griffeth-Sam conjecture. The general case of this conjecture will be proven in Sect. 6 below.

\subsection{Unitary modules and the BGS conjecture}

For $\lambda \in \mathscr{P}_{n}^{1}$, fix a positive-definite, $\mathfrak{S}_{n}$-invariant Hermitian form on the irreducible representation $S_{n}(\lambda)$. A standard argument shows that this form can be extended to a Hermitian form $(\cdot, \cdot)$ on the standard module $\Delta_{1 / e}(\lambda)$, which is $H_{1 / e}$-invariant in that $\left(y_{i} v, v^{\prime}\right)=\left(v, x_{i} v^{\prime}\right)$ for every $v, v^{\prime} \in \Delta_{1 / e}(\lambda)$ and $i=1, \ldots, n$. The simple module 
$L_{1 / e}(\lambda)$ is the quotient of $\Delta_{1 / e}(\lambda)$ by the radical of this form. In particular, $L_{1 / e}(\lambda)$ is equipped with a $H_{c}$-invariant, non-degenerate Hermitian form. We say that $L_{1 / e}(\lambda)$ is unitary if this form is positive-definite. Recall on the other hand that we have defined a $e$-unitary partition in Definition 5.5. The connection between these two notions of unitarity is given by the following result.

Theorem 5.22 [23] The Hermitian form on $L_{1 / e}(\lambda)$ is positive-definite if and only if $\lambda$ is an e-unitary partition. Thus $L_{1 / e}(\lambda)$ is unitary if and only if $\lambda \in \mathcal{F}_{n}^{1}$.

Applying the $\mathrm{KZ}$ functor to these simples, we obtain the complete set of simple unitary modules for the Hecke algebra. We emphasise that the simples labelled by $\lambda=\left(e^{k}\right)$ for some $k \geq 0$ do not survive under the $\mathrm{KZ}$ functor and so there are fewer unitary simples for the Hecke algebras. We remark that the set of simple unitary modules for the Hecke algebra coincides with that of simple calibrated representations, see e.g. [53].

Theorem 5.23 ( [54, Corollary 4.5]) For $q=\exp (2 \pi \sqrt{-1} / e)$, the simple $\mathcal{H}_{q}(n)$ module $D_{n}^{\mathbb{C}}(\lambda)$ is unitary if and only if $\lambda$ is e-restricted and $\lambda \in \mathcal{F}_{n}^{1}$.

The following result was conjectured by Berkesch-Griffeth-Sam in [65, Conjecture 4.5]. Note that it is Theorem A from the introduction.

Theorem 5.24 Let $L(\lambda)$ be a unitary, simple representation of $H_{1 / e}\left(\mathfrak{S}_{n}\right)$. Then, $L(\lambda)$ has a $B G G$ resolution of the form $C_{\bullet}(\lambda)$, where the $\ell$-th term is given by

$$
C_{\ell}(\lambda)=\bigoplus_{\substack{\mu \in \operatorname{Po}_{e}(\lambda) \\ \operatorname{hd}(\mu)=\ell}} \Delta(\mu) .
$$

At present, we are in a position to prove Theorem 5.24 under the extra assumption that $L(\lambda)$ has full support. Note that this is equivalent to saying that $\lambda \neq\left(e^{k}\right)$ for some $k \geq 0$.

Thus, assume $\lambda$ has full support. Thanks to Lemma 5.19, upon applying the equivalence $\Psi_{n}$ from Theorem 5.20, the complexes in Theorems 5.24 and 4.2 coincide. The result now follows from Corollary 4.4. The remaining case, when $\lambda$ does not have full support, will be dealt with in Sect. 6.1.

Remark 5.25 We remark that Theorem 5.22 is false when $c=a / e$ with $\operatorname{gcd}(a ; e)=1$ and $a \neq 1$. For example, if $e=n$ and $\lambda$ is the trivial partition, it follows from results of [23] that $L_{a / n}(\lambda)$ is unitary if and only if $a=1$. Nevertheless, one direction of Theorem 5.22 still holds for $a \neq 1$. Namely, it follows from [23, Theorem 5.5] that if $L_{a / e}(\lambda)$ is unitary then $\lambda \in \mathcal{F}_{n}^{1}$. Thus, Theorem 5.24 implies [65, Conjecture 4.5] in full generality.

Remark 5.26 We would like to say a few words about cyclotomic rational Cherednik algebras. Associated to the group $G(\ell, 1, n)$ there is a rational Cherednik algebra $H_{c}(G(\ell, 1, n))$, where $c=\left(c_{0}, c_{1}, \ldots, c_{\ell-1}\right)$ is now a collection of $\ell$ complex numbers. The definition of a unitary module goes through unchanged. We let $\ell=2$ and 
take the charge $c_{0}=1 / e$ and $c_{1}=0$ so that we are, essentially, working with a rational Cherednik algebra associated to the Weyl group of type $D$. We have checked using Griffeth's classification of unitary modules [30] that if $\lambda \in \mathcal{F}_{n}^{2}$ then $L(\lambda)$ is indeed a unitary module.

\section{Berkesch-Griffeth-Sam's conjecture and beyond}

In this section we use techniques from homological algebra to complete the proof of the conjecture of [65] that unitary $H_{1 / e}\left(S_{n}\right)$-modules have BGG resolutions with standard modules in a given homological degree as predicted by abacus combinatorics. The proof in earlier sections left out exactly the case that the unitary module of the Cherednik algebra does not lift a unitary module of the Hecke algebra via the KZ functor; there is at most one such unitary module for a fixed $n$. This step of the proof also highlights an interesting property of these resolutions: in a sense, they are independent of the quantum characteristic $e$. Namely, the shape of the BGG complex depends only on the weight $k$ of the block and the number $h$ of non-empty runners in the abacus of the unitary partition for $e \geq h$. Using the property of the Ringel duality that it is a perverse equivalence, we also obtain a BGG resolution of the spherical module $L$ (triv) when $e$ divides $n$.

\subsection{Changing quantum characteristics}

Having constructed a BGG resolution for any unitary module with $h<e$ columns, we proceed to relate these complexes to each other for various $e$, and to construct the complex in the special case $h=e$ for the unitary module $L\left(e^{k}\right)$. As observed in Sect. 5 , the $e$-abacus of any unitary module which is not of the form $L\left(e^{k}\right)$ will contain empty runners; removing the empty runners produces the $h$-abacus of a partition of the form $\left(h^{k}\right)$, with $h<e$ and $k$ equal to the weight of the block containing $\lambda$. So we may try using the runner removal Morita equivalences of Chuang-Miyachi which upgrade the combinatorial operation "removing runners" to an equivalence of highest weight categories [14]. Given an $e$-core partition $\rho$ and $k \in \mathbb{N}$, let $n:=|\rho|+e k$ and set

$$
\begin{aligned}
\Lambda(\rho, k) & :=\left\{\lambda \mid \lambda \in \mathscr{P}_{n}^{1}, e-\operatorname{core}(\lambda)=\rho, w(\lambda)=k\right\} \subseteq \mathscr{P}_{n}^{1}, \\
\Lambda_{h}^{+}(\rho, k) & \left.:=\left\{\lambda \in \Lambda(\rho, k) \mid \lambda \in \mathscr{P}_{n}^{1}(h)\right\} \subseteq \Lambda(\rho, k)\right\} \\
\Lambda_{h}^{-}(\rho, k) & :=\left\{\lambda \mid \lambda^{T} \in \mathscr{P}_{n}^{1}(h), e-\operatorname{core}(\lambda)=\rho^{T}, w(\lambda)=k\right\} \subseteq \Lambda\left(\rho^{T}, k\right) .
\end{aligned}
$$

Notice that the transpose map gives a bijection between the sets $\Lambda_{h}^{-}(\rho, k)$ and $\Lambda_{h}^{+}(\rho, k)$; under this map the partial ordering on the sets is reversed. Let $\mathcal{O}_{1 / e}(\rho, k)$ denote the block of category $\mathcal{O}_{1 / e}$ corresponding to $\Lambda(\rho, k)$. Note that the set $\Lambda_{h}^{-}(\rho, k)$ is co-saturated in $\Lambda\left(\rho^{T}, k\right)$ so we can consider the quotient category of $\mathcal{O}_{1 / e}\left(\rho^{T}, k\right)$ by the Serre subcategory spanned by simples whose label does not belong to $\Lambda_{h}^{-}(\rho, k)$. We denote this quotient by $\mathcal{O}_{1 / e, h}^{-}(\rho, k)$ This is a highest weight 
category, with standard objects $\Delta_{h}^{-}(v):=\pi\left(\Delta_{1 / e}(v)\right)$, where $v \in \Lambda_{h}^{-}(\rho, k)$ and $\pi: \mathcal{O}_{1 / e}\left(\rho^{T}, k\right) \rightarrow \mathcal{O}_{1 / e, h}^{-}(\rho, k)$ is the quotient functor. We remark that $\pi$ admits a left adjoint $\pi^{!}: \mathcal{O}_{1 / e, h}^{-}(\rho, k) \rightarrow \mathcal{O}_{1 / e}\left(\rho^{T}, k\right)$, and $\pi^{!}\left(\Delta^{-}(\nu)\right)=\Delta(\nu)$ for $\nu \in \Lambda_{h}^{-}(\rho, k)$.

Given $v \in \Lambda_{h}^{-}(\rho, k)$ we set $\mathcal{A}_{h}^{C M}(v):=\mathcal{A}_{h}\left(v^{T}\right)$. Let $r=\left(r_{0}, \ldots, r_{h-1}, r_{h}\right) \in$ $\mathbb{Z}_{\geq 0}^{h+1}$, and construct a partition $v^{+}$as follows. In the abacus $\mathcal{A}_{h}^{C M}(\nu)$, insert $r_{i}$ empty runners between runners $i-1$ and $i$ (so $r_{0}$ and $r_{h}$ are the number of empty runners inserted at the top and bottom of the abacus, respectively). This creates a new $e$-abacus, $\mathcal{A}$, with $e:=h+r_{0}+\cdots+r_{h}$ runners. We denote by $v^{+}$the unique partition such that $\mathcal{A}=\mathcal{A}_{e}^{C M}\left(v^{+}\right)$. We let $\rho=\varnothing^{+}$. We have a bijection

$$
\mathrm{R}: \Lambda^{-}(\varnothing, k) \rightarrow \Lambda^{-}(\rho, k)
$$

given by $\mathrm{R}: v \mapsto v^{+}$and we let $\mathrm{R}^{-1}: v \mapsto v^{-}$denote the inverse. We are now able to recall the main result of Chuang-Miyachi.

Theorem 6.1 ([14]) The categories $\mathcal{O}_{1 / h, h}^{-}(\varnothing, k)$ and $\mathcal{O}_{1 / e, h}^{-}(\rho, k)$ are equivalent as highest weight categories. Moreover, the equivalence

$$
\underline{\mathrm{R}}: \mathcal{O}_{1 / h, h}^{-}(\varnothing, k) \rightarrow \mathcal{O}_{1 / e, h}^{-}(\rho, k)
$$

sends the standard module $\Delta_{h}^{-}(v)$ to the standard module $\Delta_{h}^{-}\left(v^{\mathrm{R}}\right)$.

Note, however, that we cannot apply the above theorem directly since we are interested in the subcategories $\mathcal{O}_{1 / h, h}^{+}(\varnothing, k)$ and $\mathcal{O}_{1 / e, h}^{+}\left(\rho^{T}, k\right)$ rather than the quotient categories $\mathcal{O}_{1 / h, h}^{-}(\varnothing, k)$ and $\mathcal{O}_{1 / e, h}^{-}(\rho, k)$, where $\mathcal{O}_{1 / h, h}^{+}(\varnothing, k)$ denotes the Serre subcategory spanned by the simples whose label belongs to $\Lambda^{+}(\varnothing, k)$, and similarly for $\mathcal{O}_{1 / e, h}^{+}(\rho, k)$. Let us fix this. Following [29, Section 4], we note that the rational Cherednik algebra $H_{1 / e}:=H_{1 / e}\left(\mathfrak{S}_{n}\right)$ has finite global dimension and is isomorphic to its opposite algebra; an explicit isomorphism is given by $w \mapsto$ $w^{-1}, x \mapsto x, y \mapsto-y$. In particular, the functor $\operatorname{RHom}_{H_{1 / e}}\left(\bullet, H_{1 / e}\right)$ gives an

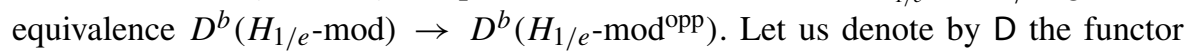
$\operatorname{RHom}_{H_{1 / e}}\left(\bullet, H_{1 / e}\right)[n]$. The following theorem summarizes various results of [29, Section 4.3.2]. We denote by $D^{b}\left(\mathcal{O}_{1 / e}\left(\mathfrak{S}_{n}\right)\right)$ the subcategory of $D^{b}\left(H_{1 / e}\right.$-mod $)$ consisting of complexes with homology in $\mathcal{O}_{1 / e}$, and by $\mathcal{O}_{1 / e}^{\Delta}$ the category of objects in $\mathcal{O}_{1 / e}$ that admit a $\Delta$-filtration.

Theorem 6.2 The functor $\mathrm{D}$ induces a derived equivalence $\mathrm{D}: D^{b}\left(\mathcal{O}_{1 / e}\left(\mathfrak{S}_{n}\right)\right) \rightarrow$ $D^{b}\left(\mathcal{O}_{1 / e}\left(\mathfrak{S}_{n}\right)^{\mathrm{opp}}\right)$ and an equivalence of exact categories $\mathrm{D}: \mathcal{O}_{1 / e}\left(\mathfrak{S}_{n}\right)^{\Delta} \rightarrow$ $\left(\mathcal{O}_{1 / e}\left(\mathfrak{S}_{n}\right)^{\Delta}\right)^{\mathrm{opp}}$. For $\lambda \vdash n, \mathrm{D}(\Delta(\lambda))=\Delta\left(\lambda^{T}\right)$ (where both sides of the equation are interpreted as complexes concentrated in degree 0$)$.

By abuse of notation, we will write D : $D^{b}\left(\mathcal{O}_{1 / e}\right) \rightarrow D^{b}\left(\mathcal{O}_{1 / e}^{\text {opp }}\right)$ for $\bigoplus_{n \geq 0}$ $\operatorname{RHom}_{H_{1 / e}}\left(\mathfrak{S}_{n}\right)\left(\bullet, H_{1 / e}\left(\mathfrak{S}_{n}\right)\right)[n]$. Let us mention a property of $\mathrm{D}$ that will be important later. The following is an immediate consequence of [42, Lemma 2.5] and the definition of a perverse equivalence [42, Section 1.4]. 
Lemma 6.3 For every $n \geq 0$, the functor $\mathrm{D}$ induces a (contravariant!) abelian autoequivalence of the category of minimally supported modules in category $\mathcal{O}_{1 / e}\left(\mathfrak{S}_{n}\right)$.

Let $\lambda \in \mathcal{F}_{n}^{1} \subseteq \mathscr{P}_{n}^{1}(h)$ be such that $\left(h^{k}\right)^{+}=\lambda$, where $k$ is the $e$-weight of $\lambda$ and $h$ the number of nonempty runners in $\mathcal{A}_{e}(\lambda)$. Define the functor $\tilde{\mathrm{R}}^{-}$via the following composition

$$
\left.\tilde{\mathrm{R}}^{-}:=\mathrm{D} \pi \underline{\mathrm{R}}^{-1} \pi \mathrm{D}: D^{b}\left(\mathcal{O}_{1 / e}(\rho, k)\right) \rightarrow D^{b}\left(\mathcal{O}_{1 / h}(\varnothing, k)\right)\right) .
$$

Each functor in the composition defining $\tilde{\mathrm{R}}^{-}$takes Vermas to Vermas, and is either an equivalence of $\Delta$-filtered categories or exact on $\Delta$-filtered categories while being an isomorphism on spaces of homomorphisms between Vermas. It follows that for $\mu \in \mathrm{Po}_{e}(\lambda), \tilde{\mathrm{R}}^{-} \Delta(\mu)=\Delta\left(\mu^{-}\right)$, and that $\tilde{\mathrm{R}}^{-}$takes a complex to a complex and sends nonzero maps to nonzero maps (however, we cannot conclude from this that $\tilde{\mathrm{R}}^{-}$takes a resolution to a resolution). Define $C_{\bullet}\left(h^{k}\right)=\tilde{\mathrm{R}}^{-}\left(C_{\bullet}(\lambda)\right)$. By construction, this is a complex whose $\ell$-th term is given by

$$
C_{\ell}\left(h^{k}\right)=\bigoplus_{\substack{\mu \in \operatorname{Po}_{e}(\lambda) \\ \operatorname{hd}(\mu)=\ell}} \Delta\left(\mu^{-}\right)=\bigoplus_{\substack{\tau \in \operatorname{Po}_{h}\left(h^{k}\right) \\ \operatorname{hd}(\tau)=\ell}} \Delta(\tau)
$$

and which has a map $\Delta(\tau) \rightarrow \Delta\left(\tau^{\prime}\right)$ whenever $\mathrm{hd}(\tau)=\ell$, hd $\left(\tau^{\prime}\right)=\ell-1$, and $\mathcal{A}_{h}(\tau)=t \mathcal{A}_{h}\left(\tau^{\prime}\right)$ for some transposition $t \in \widetilde{\mathfrak{S}}_{h} . C_{\bullet}\left(h^{k}\right)$ is a complex that looks identical to $C_{\bullet}(\lambda)$ but with the partitions $\mu$ relabeled by $\mu^{-}$, and in particular $L\left(h^{k}\right)$ is the head of $C_{0}\left(h^{k}\right)=\Delta\left(h^{k}\right)$.

We are now ready to prove Theorem 5.24, which answers [65, Conjecture 4.5] in the affirmative.

Proof of Theorem 5.24 We have already shown, in Sect. 5.10 the case when $L(\lambda)$ has full support. It remains to show the case when $\lambda=\left(e^{k}\right)$ for some $k>0$. Thus, let $n=k e$ and take $\left(e^{k}\right)$, the unique unitary partition of $n$ with $e$ columns. Choose any $e^{\prime}>e$ and any unitary partition $\lambda \in \mathcal{P}_{e^{\prime}}^{u}$ with $e$ columns and $e^{\prime}$-weight $k$. Let $C_{\bullet}(\lambda)$ be the BGG resolution of $L(\lambda)$ and apply $\tilde{\mathrm{R}}^{-}$to it. By the remarks above, $\tilde{\mathrm{R}}^{-}\left(C_{\bullet}(\lambda)\right)=C_{\bullet}\left(e^{k}\right)$ is the desired complex and $L\left(e^{k}\right)$ is the head of $C_{0}\left(e^{k}\right)$. We need to show that $C_{\bullet}\left(e^{k}\right)$ is exact except in degree 0 , where $H_{0}\left(C_{\bullet}\right)=L\left(e^{k}\right)$.

As in the proof of the $h<e$ case, if $\lambda \in \operatorname{Po}_{e}\left(e^{k}\right) \backslash\left\{\left(e^{k}\right)\right\}$, then $\lambda$ is $e$-restricted. Thus $E_{i}(L(\lambda)) \neq 0$ for some $i \in \mathbb{Z} / e \mathbb{Z}$, so if $L(\lambda)$ is a composition factor of a homology group $H_{j}\left(C_{\bullet}\right)$ then $E_{i}\left(C_{\bullet}\right)$ will fail to be exact. Similarly, it holds (by basic properties of highest weight categories) that $L\left(e^{k}\right)$ occurs exactly once in the composition series of all the $C_{j}$, when $j=0$.

Next, $E_{i}\left(L\left(e^{k}\right)\right)=0$ since $e^{k}$ has a single removable box and it is never a good removable box. Thus, it suffices to check that $E_{i}\left(C_{\bullet}\right)$ is exact for each $i \in \mathbb{Z} / e \mathbb{Z}$. This is identical to the argument used in the first case of the proof of Theorem4.2, i.e. the case where $E_{i}(L(\lambda))=0$. 
We also make the observation that resolutions of unitary modules are, in a manner of speaking, independent of $e$. Let $h$ be the number of columns of $\lambda$ and let $k$ be the $e$-weight of $\lambda$.

Corollary 6.4 Let $\lambda \in \mathcal{F}_{n}^{1}$. The shape of the BGG complex $C_{\bullet}(\lambda)$ depends only on $h, k \in \mathbb{N}$.

Proof $\tilde{\mathrm{R}}^{-}$identifies $C_{\bullet}(\lambda)$ with $C_{\bullet}\left(h^{k}\right)$, thus sends a resolution of $L(\lambda)$ to a resolution of $L\left(h^{k}\right)$.

\subsection{Ringel duality and more BGG resolutions}

We can also construct some new BGG resolutions as corollaries of Theorem 5.24 via Ringel duality. These resolutions will also be used in the study subspace arrangements in Sect. 8.1.3. The character of $L(e k)=L$ (triv) $\in \mathcal{O}_{1 / e}\left(\mathfrak{S}_{e k}\right)$ is dual to the character of $L\left(e^{k}\right)$ in the sense that its character is obtained from that of $L\left(e^{k}\right)$ by taking the transpose of each partition labelling a Verma module [22, Remark 5.1]:

$$
L(e k)=\sum_{\substack{\mu \in \operatorname{Po}_{e}\left(e^{k}\right) \\ \operatorname{hd}(\mu)=\ell}}(-1)^{\ell} \Delta\left(\mu^{T}\right) .
$$

This is every bit as much an alternating sum character formula as that of $L\left(e^{k}\right)$, so we may naturally ask whether its character formula also comes from a BGG resolution.

Let $C_{\bullet}$ be the BGG resolution of $L\left(e^{k}\right)$. We apply Ringel duality to construct a complex, $\mathrm{D}\left(C_{\bullet}\right)$, in the principal block $\mathcal{O}(\varnothing, k) \subset \mathcal{O}_{1 / e}\left(\mathfrak{S}_{e k}\right)$. The complex $\mathrm{D}\left(C_{\bullet}\right)$ is obtained from $C$ • by replacing $\Delta(\mu)$ with $\Delta\left(\mu^{T}\right)$ for all $\mu \in \operatorname{Po}_{e}\left(e^{k}\right)$ and reversing the direction of all the arrows (since $D$ is a contravariant functor which takes Vermas to Vermas). By [22], the alternating sum of the terms of $\mathrm{D}\left(C_{\bullet}\right)$ in the Grothendieck group $[\mathcal{O}(\varnothing, k)]$ coincides with the character of $L($ triv $)=L(e k)$.

Corollary $6.5 \mathrm{D}\left(C_{\bullet}\right)$ is a $B G G$ resolution of $L($ triv $)=L(e k)$.

Proof A resolution is quasi-isomorphic to the module it resolves, so in $D^{b}\left(\mathcal{O}_{1 / e}\left(\mathfrak{S}_{e k}\right)\right)$, $L\left(e^{k}\right)$ is isomorphic to its resolution $C_{\bullet}$. Since the Ringel duality $\mathrm{D}$ is a derived self-equivalence of $D^{b}\left(\mathcal{O}_{1 / e}\left(\mathfrak{S}_{e k}\right)\right)$ [29], this implies $\mathrm{D}\left(C_{\bullet}\right) \simeq \mathrm{D}\left(L\left(e^{k}\right)\right)$ in $D^{b}\left(\mathcal{O}_{1 / e}\left(\mathfrak{S}_{e k}\right)\right)$. We know that at the end of the complex we have: $\Delta(e k-1,1) \rightarrow$ $\Delta(e k) \rightarrow 0$, and so $L(e k)=\operatorname{Head}(\Delta(e k))$ must occur in the homology of $\mathrm{D}\left(C_{\bullet}\right)$. Therefore $L(e k)$ is a composition factor of $\mathrm{D}\left(L\left(e^{k}\right)\right)$.

We claim that $\mathrm{D}\left(L\left(e^{k}\right)\right)=L(e k)$. This follows from [42, Lemma 2.5] which states that $D$ is a perverse equivalence with respect to the filtration by dimensions of support: in particular, $\mathrm{D}$ is a self-equivalence of the semi-simple subcategory spanned by the minimal support modules $L(e \sigma)$. Since $\mathrm{D}^{2}=I d$, it follows that $\mathrm{D}$ must permute the minimal support simple modules $L(e \sigma), \sigma \vdash k$. We have already seen that $\mathrm{D}\left(L\left(e^{k}\right)=\mathrm{D}\left(L\left(e\left(1^{k}\right)\right)\right)\right.$ contains $L(e k)=L(e(k))$ as a composition factor; it follows that $\mathrm{D}\left(L\left(e^{k}\right)\right)=L(e k)$. 
To conclude, $\mathrm{D}\left(C_{\bullet}\right)$ is equivalent to $L(e k)$ in $D^{b}\left(\mathcal{O}_{1 / e}\left(\mathfrak{S}_{e k}\right)\right)$, where $L(e k)$ is considered as a complex concentrated in degree 0 . Hence $H_{i}\left(\mathrm{D}\left(C_{\bullet}\right)\right)=\delta_{i 0} L(e k)$, as required.

Let $\pi$ denote the quotient functor which kills the Serre subcategory generated by the simple modules $\{L(v) \mid v$ has more than $e$ rows $\}$.

Corollary $6.6 \pi \mathrm{D}\left(C_{\bullet}\right)$ is a $B G G$ resolution of $\underline{L}$ (triv) $=\underline{L}($ ek) in the quotient category $\pi\left(\mathcal{O}_{1 / e}\left(\mathfrak{S}_{k e}\right)\right)$. By adding an arbitrary configuration of $a \in \mathbb{Z}_{>0}$ empty runners to the abacus, $\mathrm{R} \pi \mathrm{D}\left(C_{\bullet}\right)$ is a $B G G$ resolution of $\mathrm{R} \underline{L}$ (triv) in $\mathrm{R} \pi\left(\mathcal{O}_{1 / e}\left(\mathfrak{S}_{k e}\right)\right)$.

Proof The quotient functor $\pi$ is exact, sends $\Delta(\mu)$ to the standard module $\underline{\Delta}(\mu)$, and sends $L(\mu)$ to the simple module $\underline{L}(\mu)$. The first claim then follows from Corollary 6.5, implying the second claim by Theorem 6.1 .

\subsection{Computation of Lie algebra and Dirac cohomology}

BGG resolutions for classical and affine Lie algebras over $\mathbb{C}$ are closely related to the computation of Lie algebra cohomology [4,28,39]. Recently, a version of Lie algebra cohomology (and homology) for rational Cherednik algebras over $\mathbb{C}$ was constructed in [36]; $\mathfrak{h}^{*}:=\bigoplus \mathbb{C} x_{i}$ plays the role of the nilradical $\mathfrak{n} \subset \mathfrak{b} \subset \mathfrak{g}$, and the complex reflection group $W$ plays the role of the Cartan subalgebra.

Theorem 6.7 Let $\lambda \in \mathcal{F}_{n}^{1} \subseteq \mathscr{P}_{n}^{1}(h)$. We have that

$$
H_{i}\left(\mathfrak{h}^{*}, L(\lambda)\right)=\bigoplus_{\substack{\mu \in \operatorname{Po}_{e}(\lambda) \\ \operatorname{hd}(\mu)=i}} S_{n}(\mu) .
$$

Proof This follows immediately from our main theorem and [36, Proposition 6.1].

Likewise, if $L(\lambda) \in \mathcal{O}_{c}(G(\ell, 1, n))$ where $c$ corresponds to the rank $e$ and charge $\underline{s}=\left(\kappa_{1}, \kappa_{2}, \ldots, \kappa_{\ell}\right) \in \mathbb{Z}^{\ell}$ for the Fock space, and $\lambda \in \mathcal{F}_{n}^{\ell}$, then

$$
H_{i}\left(\mathfrak{h}^{*}, L(\lambda)\right)=\bigoplus_{\substack{\mu \leqslant \lambda \\ \ell(\mu)=i}} S_{n}(\mu) .
$$

This also computes the Lie algebra cohomology $H^{i}\left(\mathfrak{h}^{*}, L(\lambda)\right)$. Indeed, by Poincaré duality (cf. [36, Proposition 2.7]), we get

$$
H^{i}\left(\mathfrak{h}^{*}, L(\lambda)\right)=H_{n-i}\left(\mathfrak{h}^{*}, L(\lambda)\right) \otimes \wedge^{n} \mathfrak{h},
$$

where $n:=\operatorname{dim} \mathfrak{h}$. A consequence of the computation of Lie algebra cohomology for unitary modules admitting a BGG resolution is that this immediately gives the computation of the Dirac cohomology $H_{D}(L(\lambda))$. This is defined as the usual Dirac cohomology, where the Dirac operator $D \in H_{1 / e}\left(\mathfrak{S}_{n}\right) \otimes \mathfrak{c}$ has been constructed in 
[15]. Here $\mathfrak{c}$ is the Clifford algebra associated to $\bigoplus \mathbb{C} x_{i} \oplus \bigoplus \mathbb{C} y_{j}$ with its natural nondegenerate bilinear form $\left(x_{i}, y_{j}\right)=\delta_{i j}$. For a module $M \in \mathcal{O}_{1 / e}\left(\mathfrak{S}_{n}\right)$, the algebra $H_{1 / e}\left(\mathfrak{S}_{n}\right) \otimes \mathfrak{c}$ acts on the space $M \otimes \wedge \bullet \mathfrak{h}$, and the Dirac cohomology is defined to be, as usual, $\operatorname{ker}(D) / \operatorname{ker}(D) \cap \operatorname{im}(D)$. This is a representation of $\widetilde{W}$, a certain double-cover of the group $W$. Then, by [36, Theorem 5.1], $H_{D}(L(\lambda))=\bigoplus_{\mu \leq \lambda} S_{n}(\mu) \otimes \chi$, where $\chi$ is a 1-dimensional character of the double cover $\widetilde{W}$. We refer to [36] for details.

\section{The Mullineux map on unitary simple modules}

We first recall the Mullineux involution on the quiver Hecke algebra of the symmetric group: Let $\mathrm{M}$ denote the $H_{n}$-automorphism determined by

$$
\mathrm{M}: e\left(i_{1}, i_{2}, \ldots, i_{n}\right) \mapsto e\left(-i_{1},-i_{2}, \ldots,-i_{n}\right) \quad \mathrm{M}: \psi_{r} \mapsto \psi_{r} \quad \mathrm{M}: y_{k} \mapsto y_{k}
$$

for $0 \leq k \leq n$ and $0 \leq r<n$ and $\underline{i}=\left(i_{1}, \ldots, i_{n}\right) \in(\mathbb{Z} / e \mathbb{Z})^{n}$, where $\psi_{r}$ and $y_{k}$ are generators of $H_{n}$ as given in Remark 1.19.

Given a simple module $D_{n}(\lambda)$, we let $D_{n}(\lambda)^{\mathrm{M}}$ denote the module with the same underlying vector space but with the multiplication defined by twisting the action with the involution $\mathrm{M}$. The relationship between these two simples was the subject of a conjecture of Mullineux [48]. The combinatorics of this relationship is fiendishly complicated in general and is only understood on the level of the labels of simple modules. The purpose of this section is to examine the effect of the Mullineux map on the simple modules $D_{n}(\lambda)$ for $\lambda \in \mathcal{F}_{n}^{1}$. We show that the set of these simples is preserved under the Mullineux involution. Moreover, we construct an explicit Mullineux isomorphism in terms of the bases and representing matrices of these simples given in Theorems 4.2 and 4.3 - we remark that this is the time the Mullineux isomorphism has been explicitly constructed (outside of the trivial semisimple case). Furthermore we shall see that the Mullineux combinatorics drastically simplifies on unitary $e$-regular partitions $\lambda$ and that we can easily compute $\mathrm{M}(\lambda)$ on the $e$-abacus of $\lambda$. We define the unitary branching graph, $\mathcal{Y}$, to have vertices on level $k$ given by

$$
\mathcal{Y}_{k}=\left\{\lambda \mid \lambda \text { is } e-\text { restricted and } \lambda \in \mathcal{F}_{k}^{1}\right\}
$$

and edges connecting levels $k$ and $k+1$ given by

$$
\mathcal{E}_{k, k+1}=\left\{\lambda \rightarrow \mu \mid \lambda \in \mathcal{Y}_{k}, \mu \in \mathcal{Y}_{k+1} \text { and } \lambda=\mu-\square \text { for } \square \text { a good node }\right\}
$$

We first discuss how the abaci of an $e$-core $\rho$ and its transpose $\rho^{t}$ are obtained from one another when $\rho$ has at most $e-1$ columns. Recall the basics of abaci from Sect. 5.1. First, note that if $\rho$ has at most $h<e$ columns then $\rho^{t}$ has at most $e-h$ columns. Now, let $\mathcal{A}_{e}^{h}(\rho)$ denote the $e$-abacus of $\rho$ written with $h$ beads, and perform the following procedure on it: (1) swap the empty spots and the beads in the first column (so that the resulting abacus has $e-h$ beads), then (2) flip this abacus upside down. The resulting abacus, $\mathcal{A}_{e}^{e-h}\left(\rho^{t}\right)$, is the $e$-abacus of $\rho^{t}$ written with $e-h$ beads. 
Definition 7.1 Let $\lambda \in \mathcal{F}_{n}(h)$ for some $1 \leq h<e$ and let $\rho$ be the $e$-core of $\lambda$. Write $w(\lambda)=(e-h) q+r$ for some $q \geq 0,0 \leq r<e-h$. Define $\lambda_{\mathrm{M}}$ to be the partition with abacus obtained from $\mathcal{A}_{e}^{e-h}\left(\rho^{t}\right)$ by moving the bottom $r$ beads $(q+1)$-units to the right, and the top $e-h-r$ beads $q$ units to the right.

Proposition 7.2 If $\lambda \in \mathcal{Y}_{n}$, then $\lambda_{\mathrm{M}} \in \mathcal{Y}_{n}$. Specifically: in the case $\lambda=\rho$, we have $\rho_{\mathrm{M}}=\rho^{t}$. Otherwise, we have $\lambda_{\mathrm{M}} \in \mathcal{F}_{n}(e-h)$.

Proof If $\lambda=\rho$ is an $e$-core, then $w(\lambda)=0$ and algorithm just stops after the step where we take the transpose of $\rho$. The abacus $\mathcal{A}_{e}^{e-h}\left(\rho^{t}\right)$ clearly satisfies the criterion for unitarity (Definition 5.5) since all of its beads are concentrated in the first column. If $w(\lambda)>0$, so $\lambda$ is not an $e$-core, we must move the bottom-most bead of $\mathcal{A}_{e}^{e-h}\left(\rho^{t}\right)$ at least one unit to the right to obtain $\mathcal{A}_{e}^{e-h}\left(\lambda_{\mathrm{M}}\right)$. This guarantees that $\mathcal{A}_{e}^{e-h}\left(\lambda_{\mathrm{M}}\right)$ does not start with a bead, and since $\mathcal{A}_{e}^{e-h}\left(\lambda_{\mathrm{M}}\right)$ has $e-h$ beads, we conclude that $\lambda_{\mathrm{M}}$ has precisely $e-h$ columns. Finally, by construction, $\lambda_{\mathrm{M}}$ satisfies the conditions of Definition 5.5.

Example 7.3 Let $e=5, h=2, \lambda=\left(2^{28}, 1^{3}\right), w(\lambda)=11$. We obtain $\lambda_{\mathrm{M}}=\left(3^{19}, 1^{2}\right)$ as follows:

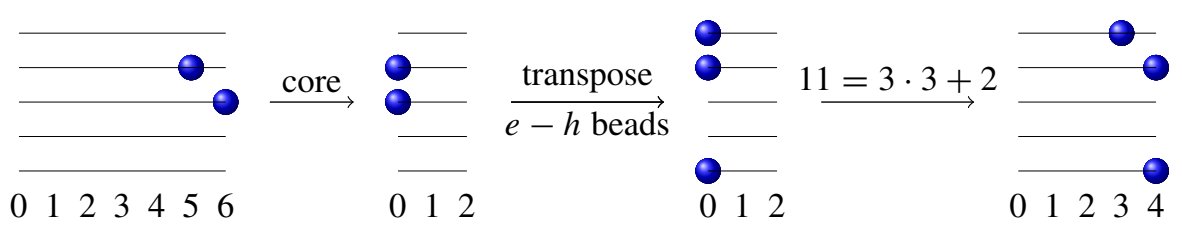

Theorem 7.4 The map $\mathrm{M}: \mathcal{Y}_{k} \rightarrow \mathcal{Y}_{k}$ for $k \geq 0$ is a well-defined graph involution. Given

$$
\mathrm{S}=\left(\lambda^{(0)} \stackrel{r_{1}}{\rightarrow} \lambda^{(1)} \stackrel{r_{2}}{\rightarrow} \ldots \stackrel{r_{n}}{\rightarrow} \lambda^{(n)}\right)
$$

we let $\mathrm{s}_{\mathrm{M}}$ denote the path

$$
\mathrm{s}_{\mathrm{M}}=\left(\lambda_{\mathrm{M}}^{(0)} \stackrel{-r_{1}}{\longrightarrow} \lambda_{\mathrm{M}}^{(1)} \stackrel{-r_{2}}{\longrightarrow} \ldots \stackrel{-r_{n}}{\longrightarrow} \lambda_{\mathrm{M}}^{(n)}\right)
$$

We have that $D_{n}\left(\lambda_{\mathrm{M}}\right) \cong\left(D_{n}(\lambda)\right)^{\mathrm{M}}$ and that the isomorphism is determined by $: c_{\mathrm{S}} \mapsto$ $c_{\mathrm{S}_{M}}$.

Proof The Mullineux involution M is characterized as the unique involution on $e$ regular partitions mapping $\emptyset$ to $\emptyset$ and such that $\mathrm{M}\left(\tilde{f}_{i}(\lambda)\right)=\tilde{f}_{-i}(\mathrm{M}(\lambda))[2,26,38]$. We want to identify $\lambda_{\mathrm{M}}$ with $\mathrm{M}(\lambda)$ for all vertices $\lambda$ of $\mathcal{Y}$. By construction we have that $\lambda_{\mathrm{M}}$ is also vertex of $\mathcal{Y}_{k}$ whenever $\lambda$ is, and that $\left(\lambda_{\mathrm{M}}\right)_{\mathrm{M}}=\lambda$. It is clear that $\emptyset_{\mathrm{M}}=\emptyset$. Thus if $i \in\{0, \ldots, e-1\}$ is such that $\tilde{f}_{i}(\lambda) \in \mathcal{F}_{n}^{1}$, we need to show that

$$
\left(\tilde{f}_{i}(\lambda)\right)_{\mathrm{M}}=\tilde{f}_{-i}\left(\lambda_{\mathrm{M}}\right)
$$


We remark that, if $\lambda \in \mathcal{F}_{n}^{1}$, then $\tilde{f}_{i}(\lambda)$ adds the leftmost addable box of content residue $i$, if any. In order to keep track of the action of $\tilde{f}_{i}$ on abaci, we follow the conventions of Remark 5.4, so we label the runners of an $e$-abacus with $h$ beads, at most one bead per runner, from bottom-to-top by $h-1, h-2, \ldots, 1,0, e-1, \ldots, h$. This is done so that the labels of the runners correspond nicely to the contents of addable/removable boxes. Note that the labeling of runners changes in the process of constructing $\lambda_{\mathrm{M}}$, when $\mathcal{A}_{e}(\rho)$ with $h$ beads is replaced by $\mathcal{A}_{e}\left(\rho^{t}\right)$ with $e-h$ beads. The abacus $\mathcal{A}_{e}(\lambda)$ has a bead (resp. empty space) on runner $i$ if and only if $\mathcal{A}_{e}\left(\lambda_{\mathrm{M}}\right)$ has an empty space (resp. bead) on runner $-i-1$. Finally, observe that if the top runner is labeled $m$ in these conventions, that $\tilde{f}_{m}$ increases the weight $w$ of a partition by at most 1 but all $\tilde{f}_{i}, i \neq m$, do not increase the weight. Now set $\hat{\rho}$ to be the core of $\tilde{f}_{i}(\lambda)$. We consider two cases.

Case 1. $w\left(\tilde{f}_{i}(\lambda)\right)=w(\lambda)$. So either $i \neq h$ or $i=h$ and $\lambda$ is a core. In the latter case, $\tilde{f}_{i}(\lambda)$ is also a core, and both $\left(\tilde{f}_{i}(\lambda)\right)_{\mathrm{M}}$ and $\tilde{f}_{-i}\left(\lambda_{\mathrm{M}}\right)$ coincide with the transpose of $\tilde{f}_{i}(\lambda)$. In the former case, the abaci $\mathcal{A}_{e}(\rho)$ and $\mathcal{A}_{e}(\hat{\rho})$ coincide on all runners except those labeled by $i$ and $i-1$. Thus, $\mathcal{A}_{e}\left(\rho^{t}\right)$ and $\mathcal{A}_{e}\left(\hat{\rho}^{t}\right)$ only differ on runners $-i-1$ and $-i: \mathcal{A}_{e}\left(\hat{\rho}^{t}\right)$ has a bead on runner $-i-1$ and an empty space on runner $-i$, while the opposite is true for $\mathcal{A}_{e}\left(\rho^{t}\right)$. Thus, $\left(\tilde{f}_{i}(\lambda)\right)_{\mathrm{M}}$ is obtained from $\lambda_{\mathrm{M}}$ by sliding the bead on runner $-i$ up runner $-i-1$. But this is exactly how we obtain $\tilde{f}_{-i}\left(\lambda_{\mathrm{M}}\right)$ from $\lambda_{\mathrm{M}}$. We are done in this case.

Case 2. $w\left(\tilde{f}_{i}(\lambda)\right)=w(\lambda)+1$. So $i=h$, and the abacus of $\tilde{f}_{i}(\lambda)$ is obtained from that of $\lambda$ by moving the bead on the top runner (labeled $h$ ) down to the bottom runner (labeled $h-1$ ) and then one unit right. Just as in the first case, the abaci $\mathcal{A}_{e}\left(\rho^{t}\right)$ and $\mathcal{A}_{e}\left(\hat{\rho}^{t}\right)$ only differ on runners $-h$ and $-h-1$. Note that these are the top and bottom runners of the abacus, respectively. Write division with remainder $w=w(\lambda)=(e-h) q+r$, so that $\lambda_{\mathrm{M}}$ is obtained from $\mathcal{A}_{e}\left(\rho^{t}\right)$ by moving the bottom $r$ beads $q+1$ units to the right, and the remainder $e-h-r$ beads $q$ units to the right. We have a subdivision into two further cases.

Case 2.1. $r<e-h-1$. So $w+1=(e-h) q+(r+1)$ is division with remainder, and $\left(\tilde{f}_{i}(\lambda)\right)_{\mathrm{M}}$ is obtained from $\mathcal{A}_{e}\left(\hat{\rho}^{t}\right)$ by moving the bottom $r+1$ beads $q+1$ units to the right, and the remaining beads $q$ units to the right. Note that the beads $2, \ldots, r+1$ of $\mathcal{A}_{e}\left(\hat{\rho}^{t}\right)$ coincide with the beads $1, \ldots, r$ of $\mathcal{A}_{e}\left(\rho^{t}\right)$. Thus, $\left(\tilde{f}_{i}(\lambda)\right)_{\mathrm{M}}$ is obtained from $\lambda_{\mathrm{M}}$ by taking the bead in the top runner, moving it down to the bottom runner and sliding one unit to the right. This is precisely $\tilde{f}_{-i}\left(\lambda_{\mathrm{M}}\right)$.

Case 2.2. $r=e-h-1$. So $w+1=(e-h)(q+1)$. Here, $\left(\tilde{f}_{i}(\lambda)\right)_{\mathrm{M}}$ is obtained from $\mathcal{A}_{e}\left(\hat{\rho}^{t}\right)$ by moving all beads $q+1$ units to the right, while $\lambda_{\mathrm{M}}$ is obtained from $\mathcal{A}_{e}\left(\rho^{t}\right)$ by moving all beads $q+1$ units to the right, except the one in the top runner, that we only move $q$ units to the right. So we see that, again, $\left(f_{i}(\lambda)\right)_{\mathrm{M}}$ is obtained from $\lambda_{\mathrm{M}}$ by taking the bead in the top runner, moving it down to the bottom runner and sliding one unit to the right. So $\left(\tilde{f}_{i}(\lambda)\right)_{\mathrm{M}}=\tilde{f}_{-i}\left(\lambda_{\mathrm{M}}\right)$.

This proves that the involution $\mathcal{Y} \rightarrow \mathcal{Y}$ given by $\lambda \mapsto \lambda_{\mathrm{M}}$ coincides with the Mullineux involution restricted to $\mathcal{Y}$. Now, the bases of $D_{n}(\lambda)$ for $\lambda \in \mathcal{F}_{n}^{1}$ are given by the paths in the unitary branching graph terminating at said vertices. By Theorem 4.3 we can match up these bases through the action of the idempotents under the twisting by the Mullineux map (see equation (7.1)). 
Fig. 6 A pair of tableaux $\mathrm{t} \in \operatorname{Std}_{7}\left(3^{10}, 2^{4}\right)$ and $\mathrm{t}_{\mathrm{M}} \in \operatorname{Std}_{7}\left(4^{8}, 1^{3}\right)$ indexing basis elements swapped under the isomorphism $D_{35}\left(3^{10}, 2^{4}\right)^{\mathrm{M}} \cong D_{35}\left(4^{8}, 1^{3}\right)$

\begin{tabular}{|c|c|}
\hline 12 & 23 \\
\hline$4 \longdiv { 8 }$ & $\begin{array}{r}89 \\
\end{array}$ \\
\hline \begin{tabular}{l|l}
5 & 11
\end{tabular} & $1 \longdiv { 1 5 }$ \\
\hline 612 & \begin{tabular}{l|l}
2 & 18
\end{tabular} \\
\hline \begin{tabular}{l|l}
7 & 13 \\
\end{tabular} & \begin{tabular}{l|l}
3 & 19
\end{tabular} \\
\hline 1014 & $4 \sqrt{20}$ \\
\hline 1617 & 721 \\
\hline 2223 & 324 \\
\hline$2 5 \longdiv { 2 9 }$ & 930 \\
\hline 2632 & \\
\hline $27 \mid 33$ & \\
\hline 2834 & \\
\hline 3135 & \\
\hline
\end{tabular}

Example 7.5 Let $e=7$. We have that $\mathrm{M}\left(3^{10}, 2^{4}\right)=\left(4^{8}, 1^{3}\right)$. We depict these partitions, and the manner in which they can constructed via adding rim 7-hooks in Figure 6. Furthermore, we provide an example of $t \in \operatorname{Std}_{7}\left(3^{10}, 2^{4}\right)$ and $t_{M} \in \operatorname{Std}_{7}\left(4^{8}, 1^{3}\right)$. Note that the map on the level of tableau preserves the rim hooks drawn in the two diagrams!!

\section{Graded free resolutions of algebraic varieties, Betti numbers, and Castelnuovo-Mumford regularity}

We now consider the consequences of our results for computing minimal resolutions of linear subspace arrangements. Easy examples of ideals whose resolutions we compute include the braid arrangements of type $A$ and type $D$. Such minimal resolutions are difficult to compute geometrically [40]. As a consequence, we prove a combinatorial formula for the Betti numbers of the ideal of the $m$-equals arrangement predicted in [65]. We also calculate the Castelnuovo-Mumford regularity for the coordinate ring of these arrangements, a notoriously difficult problem in general (see [16,57]).

It is pointed out in [20] that BGG resolutions via parabolic Verma modules for Lie algebras can be used to provide commutative algebra resolutions of determinantal ideals by viewing the coordinate ring as a unitarizable highest weight module. We employ our Cherednik algebra resolutions in an analogous fashion. The first of these commutative algebra resolutions, given in Sect. 8.1.1, was predicted in [65] and concerns the smallest ideal, $I_{e, 1, n}$, of the polynomial representation (this is the vanishing ideal of the subspace arrangement, $X_{e, 1, n}$, consisting of $e$ equal coordinates for $e \leq n$ ). We then provide a cyclotomic generalisation of this resolution in Sect. 8.1.2. The third resolution, given in Sect. 8.1.3, concerns the smallest quotient, $\mathbb{C}\left[X_{e, k, n}\right]$, of the polynomial representation (this is the coordinate ring of the subspace arrangement, $X_{e, k, n}$, consisting of $k$ clusters of $e$ equal coordinates for $k e=n$ ); the ideal vanishing on this 
space was studied in [65], however since neither this ideal nor its quotient is unitary (in general) the authors did not predict any resolution arising via Cherednik algebras.

\subsection{Commutative algebra}

Let us discuss the consequences that the existence of the BGG resolution has for the study of graded modules over $\mathbb{C}\left[x_{1}, \ldots, x_{n}\right]=\mathbb{C}[\underline{x}]$. First of all, for every $\mu \vdash n$, the standard module $\Delta_{1 / e}(\mu)$ is free as a $\mathbb{C}[\underline{x}]$-module. So the resolution $C_{\bullet}(\lambda)$ is, in fact, a free resolution of $L_{1 / e}(\lambda)$ when we view all involved modules as $\mathbb{C}[\underline{x}]$-modules.

An observation now is that every module in category $\mathcal{O}_{1 / e}\left(\mathfrak{S}_{n}\right)$ automatically acquires a grading compatible with the usual grading on $\mathbb{C}[\underline{x}]$, as follows. Consider the deformed Euler element ${ }^{1} \mathrm{eu}:=\frac{1}{2} \sum_{i=1}^{n} x_{i} y_{i}+y_{i} x_{i} \in H_{1 / e}$. This is a grading element of $H_{1 / e}$ in the sense that [eu, $x_{i}$ ] $=x_{i}$, [eu, $y_{i}$ ] $=-y_{i}$, and [eu, $\left.w\right]=0$ for $w \in \mathfrak{S}_{n}$. Any module in category $\mathcal{O}_{1 / e}\left(\mathfrak{S}_{n}\right)$ is now graded by generalized eigenspaces for eu:

$$
M=\bigoplus_{a \in \mathbb{C}} M_{a}, M_{a}:=\left\{m \in M:(\mathrm{eu}-a)^{k} m=0 \text { for } k \gg 0\right\} .
$$

Note that, since the grading on $M$ was defined using an element of $H_{1 / e}$, every morphism in category $\mathcal{O}_{1 / e}\left(\mathfrak{S}_{n}\right)$ has degree 0 . In particular, this grading is different from the grading of objects in $\mathcal{O}_{1 / e}\left(\mathfrak{S}_{n}\right)$ that has been used so far in this paper. The grading by generalized eigenspaces of eu, however, is better-suited for the purposes of commutative algebra.

A priori, $M \in \mathcal{O}_{1 / e}\left(\mathfrak{S}_{n}\right)$ is only $\mathbb{C}$-graded, but in our case we can do better. Since [eu, $w]=0$ for $w \in \mathfrak{S}_{n}$, eu may be seen as an endomorphism of the $\mathfrak{S}_{n}$-module $S_{n}(\tau) \cong 1 \otimes S_{n}(\tau) \subseteq \mathbb{C}[\underline{x}] \otimes \tau=\Delta_{1 / e}(\tau)$. Thus, eu acts by a scalar $c_{\tau}$ on $S_{n}(\tau)$, and by the definition of $\Delta_{1 / e}(\tau)$ we get that $\Delta_{1 / e}(\tau)_{a} \neq 0$ if and only if $a=c_{\tau}+k$ for some $k \in \mathbb{Z}_{\geq 0}$. Moreover,

$$
\Delta_{1 / e}(\tau)_{c_{\tau}+k}=\mathbb{C}[\underline{x}]_{k} \otimes S_{n}(\tau)
$$

where $\mathbb{C}[\underline{x}]_{k}$ denotes the subspace of homogeneous polynomials of degree $k$ in the variables $x_{1}, \ldots, x_{n}$. We will write $\mathbb{C}\left[\underline{x]} \otimes S_{n}(\lambda)\right.$ to refer to the $\mathbb{C}[\underline{x}]$-module $\Delta_{1 / e}(\lambda)\left[c_{\lambda}\right]$, where the brackets denote the usual grading shift. Thus, $\mathbb{C}[\underline{x}] \otimes S_{n}(\lambda)$ is $\mathbb{Z}_{\geq 0}$-graded, and $\left(\mathbb{C}[\underline{x}] \otimes S_{n}(\lambda)\right)_{k}=\mathbb{C}[\underline{x}]_{k} \otimes S_{n}(\lambda)$.

Now consider the resolution of the graded $\mathbb{C}[\underline{x}]$-module $L_{1 / e}(\lambda)\left[c_{\lambda}\right]$, where the $i$-th term of the complex is given by

$$
\bigoplus_{\substack{\mu \in \operatorname{Po}_{e}(\lambda) \\ \operatorname{hd}(\mu)=i}}\left(\mathbb{C}[\underline{x}] \otimes S_{n}(\mu)\right)\left[c_{\lambda}-c_{\mu}\right]
$$

We remark that, since $\lambda$ and $\mu$ belong to the same block of category $\mathcal{O}_{1 / e}\left(\mathfrak{S}_{n}\right), c_{\lambda}-c_{\mu}$ is actually an integer. Of course, this is the same as the BGG resolution $C_{\bullet}(\lambda)$, but

\footnotetext{
1 We remark that our Euler element 'eu'differs from the one used in [65] by the constant $n(e-n+1) / 2 e$.
} 
we write it in this way to emphasize that we are only interested in the $\mathbb{C}[\underline{x}]$-module structure. By abuse of notation, we also denote this complex by $C_{\bullet}(\lambda)$. Note that $\left(\mathbb{C}[\underline{x}] \otimes S_{n}(\mu)\right)\left[c_{\lambda}-c_{\mu}\right]=\Delta_{1 / e}(\mu)\left[c_{\lambda}\right]$, from where it follows that all the maps in the complex have degree 0 as maps of graded $\mathbb{C}[\underline{x}]$-modules. In particular, $C_{\bullet}(\lambda)$ is a graded-free resolution of $L_{1 / e}(\lambda)\left[c_{\lambda}\right]$.

The value of $c_{\lambda}$ can be expressed in terms of the content of the boxes of $\lambda$, namely

$$
c_{\lambda}=\frac{n}{2}-\frac{1}{e} \sum_{\square \in \lambda} \operatorname{column}(\square)-\operatorname{row}(\square)
$$

It follows from Sect. 5.6 or from Lemma 5.19 that if $\mathrm{hd}(\mu)<\operatorname{hd}(v)$ then $c_{\mu}<c_{\nu}$. In particular, when viewing the differential in the resolution $C_{\bullet}(\lambda)$ as matrices with coefficients in $\mathbb{C}[\underline{x}]$, no nonzero entry of the differential is a degree 0 element of $\mathbb{C}[\underline{x}]$. It follows immediately that:

Lemma 8.1 The complex $C_{\bullet}(\lambda)$ is a minimal graded free resolution of $L_{1 / e}(\lambda)\left[c_{\lambda}\right]$.

Lemma 8.1 implies a combinatorial formula for computing many interesting invariants of the module $L_{1 / e}(\lambda)\left[c_{\lambda}\right]$. In the rest of this section, if $L_{1 / e}(\lambda)$ is unitary we write:

$$
n:=|\lambda|, \quad k:=e \text {-weight }(\lambda), \quad h:=\# \operatorname{columns}(\lambda)
$$

Recalling the basics of abaci in Sect. 5.1 this means that the abacus $\mathcal{A}_{e}(\lambda)$ has $h$ nonempty runners and there are $k$ vacant spaces in $\mathcal{A}_{e}(\lambda)$ with some bead to their right.

Proposition 8.2 Suppose $L_{1 / e}(\lambda)$ is unitary. Then,

(1) $\beta_{i, j}=\sum_{\begin{array}{c}\mu \in \operatorname{Po}_{e}(\lambda) \\ c_{\lambda}-c_{\mu}=-j \\ \operatorname{hd}(\mu)=i\end{array}} \operatorname{dim}\left(S_{n}(\mu)\right)$,

(2) $\operatorname{pdim}\left(L_{1 / e}(\lambda)\right)=(h-1) k$,

(3) $\operatorname{depth}\left(L_{1 / e}(\lambda)\right)=n-(h-1) k$

where $\beta_{i, j}$ denotes the $(i, j)$-graded Betti number of $L_{1 / e}(\lambda)\left[c_{\lambda}\right]$, and pdim stands for the projective dimension as a graded $\mathbb{C}[\underline{x}]$-module.

Proof Statement (1) is clear from the form of the resolution $C_{\bullet}(\lambda)$. The maximal homological degree of a partition in $\operatorname{Po}_{e}(\lambda)$ is acquired by sliding all the beads to the left and then sliding the highest bead $k$ spaces to the right. (2) follows from here. Finally, by the Auslander-Buchsbaum formula, (3) is equivalent to (2).

Another consequence of Lemma 8.1 and the fact that the function $c_{\lambda}$ is strictly increasing on homological degrees, is the computation of the Castelnuovo-Mumford regularity of the module $L_{1 / e}(\lambda)\left[c_{\lambda}\right]$. Recall that, by definition, the regularity of a module $M$ is

$$
\operatorname{reg}(M):=\max \left\{j: \text { there exists } i \text { such that } \beta_{i, i+j}(M) \neq 0\right\}
$$


In other words, for a minimal graded-free resolution $C_{\bullet}$ of $M$, for each $i=$ $0, \ldots, \operatorname{pdim}(M)$, let $n_{i}$ be the maximum degree of a generator of $C_{i}$, and $m_{i}:=n_{i}-i$. Then, $\operatorname{reg}(M)=\max _{i}\left\{m_{i}\right\}$. The Castelnuovo-Mumford regularity is a measure of the computational complexity of the module $M$ and it is, in general, incredibly difficult to compute, cf. [16,57].

Proposition 8.3 Suppose $L_{1 / e}(\lambda)$ is unitary. Let $\mu_{0} \in \mathrm{Po}_{e}(\lambda)$ be obtained by, first, sliding all beads of $\mathcal{A}_{e}(\lambda)$ to the left, and then, sliding the upmost beat $k$ spaces to the right. Then,

$$
\operatorname{reg}\left(L_{1 / e}(\lambda)\left[c_{\lambda}\right]\right)=\left(c_{\mu_{0}}-c_{\lambda}\right)-(h-1) k
$$

Proof As in the paragraph above the statement of the proposition, let us denote by $n_{i}$ the maximum degree of a generator of $C(\lambda)_{i}$, and $m_{i}:=n_{i}-i$. Note that $n_{i}:=\max \left\{c_{\mu}-\right.$ $\left.c_{\lambda}: \mu \in \operatorname{Po}_{e}(\lambda), \operatorname{hd}(\mu)=i\right\}$. Since the $c$-function is increasing in homological degree, the sequence $\left(n_{i}\right)$ is increasing and therefore the sequence $\left(m_{i}\right)$ is nondecreasing. So the regularity of $L_{1 / e}(\lambda)\left[c_{\lambda}\right]$ is $m_{\operatorname{pdim}\left(L_{1 / e}(\lambda)\right)}$. Since $\operatorname{pdim}\left(L_{1 / e}(\lambda)\right)=(h-1) k$, the result follows.

Example 8.4 Consider $e=5, n=15$ and $\lambda=\left(3^{4}, 2,1\right)$. Then, $\operatorname{pdim} L_{1 / e}(\lambda)=4$, so $L_{1 / e}(\lambda)$ is not Cohen-Macaulay and a minimal graded-free resolution of $L_{1 / e}(\lambda)\left[c_{\lambda}\right]$ is

$$
\begin{aligned}
0 & \rightarrow\left(3,2,1^{10}\right)[-9] \rightarrow\left(3,2^{2}, 1^{7}\right)[-5] \rightarrow\left(3,2^{6}\right)[-3] \oplus\left(3^{3}, 1^{6}\right)[-3] \\
& \rightarrow\left(3^{3}, 2^{2}, 1^{2}\right)[-1] \rightarrow\left(3^{4}, 2,1\right) \\
& \rightarrow L_{1 / e}(\lambda)\left[c_{\lambda}\right] \rightarrow 0
\end{aligned}
$$

where for brevity, we write $\mu[d]$ in place of $\left(\mathbb{C}[\underline{x}] \otimes S_{n}(\mu)\right)[d]$. From the resolution, we see that $\operatorname{reg}\left(L_{1 / e}(\lambda)\left[c_{\lambda}\right]\right)=5$.

\subsubsection{The $e$-equals ideal}

We examine these results in the situation where the modules $L_{1 / e}(\lambda)$ have a clear geometric meaning. The representation theoretic import of $X_{e, 1, n}$ was first noticed and explained in [65]. Resolutions of the ideals vanishing on these subspace arrangements are given by BGG resolutions of the corresponding unitary module for $H_{1 / e}\left(\mathfrak{S}_{n}\right)$.

Let $n=(e-1) p+q$, with $0 \leq q<e-1$. Consider the partition $\lambda=\left((e-1)^{p}, q\right)$ of $n$. Note that the $e$-abacus of $\lambda$ has exactly one empty runner, and the module $L_{1 / e}(\lambda)$ is unitary. In fact, it follows from [62] that $L_{1 / e}(\lambda)$ is isomorphic to the socle of the polynomial representation

$$
\Delta_{1 / e}(\text { triv }) \cong \mathbb{C}\left[x_{1}, x_{2}, \ldots, x_{n}\right]
$$

which by [23, Theorem 5.10] coincides with the $e$-equals ideal $I_{e, 1, n}$ of functions vanishing on the set

$$
X_{e, 1, n}:=\mathfrak{S}_{n}\left\{\left(z_{1}, \ldots, z_{n}\right) \in \mathbb{C}^{n}: z_{1}=\cdots=z_{e}\right\} .
$$


Note that $X_{e, 1, n}$ is an arrangement of $\left(\begin{array}{l}n \\ e\end{array}\right)$ linear subspaces of $\mathbb{C}^{n}$, each of dimension $n-e+1$. When $e=2, X_{2,1, n}$ is nothing but the braid arrangement in $\mathbb{C}^{n}$, which consists of the reflection hyperplanes for the action of $\mathfrak{S}_{n}$ on $\mathbb{C}^{n}$. Let us give a set of generators for the ideal $I_{e, 1, n}$, following $[23,24]$. Consider the partition $\lambda^{T}=\left((p+1)^{q}, p^{e-1-q}\right)$, which has exactly $e-1$ parts. Now consider the polynomial

$p_{\lambda^{T}}\left(x_{1}, \ldots, x_{n}\right)=V\left(x_{1}, \ldots, x_{\lambda_{1}^{T}}\right) V\left(x_{\lambda_{1}^{T}+1}, \ldots, x_{\lambda_{1}^{T}+\lambda_{2}^{T}}\right) \cdots V\left(x_{\lambda_{1}^{T}+\cdots+\lambda_{e-2}^{T}+1}, \ldots, x_{n}\right)$

where $V\left(x_{1}, \ldots, x_{k}\right)$ is the Vandermonde determinant $\prod_{i<j}\left(x_{i}-x_{j}\right)$. Then, the ideal $I_{e, 1, n}$ is generated by the $\mathfrak{S}_{n}$ images of the polynomial $p_{\lambda^{T}}$.

Since $L_{1 / e}(\lambda)$ and $L_{1 / e}$ (triv) lie in the same block of category $\mathcal{O}_{1 / e}$, the weight of the partition $\lambda$ is $k=\lfloor n / e\rfloor$. Thus, as was observed in [65], the projective dimension of the algebra of functions $\mathbb{C}\left[X_{e, 1, n}\right]=\mathbb{C}\left[x_{1}, \ldots, x_{n}\right] / I_{e, 1, n}$ is $\operatorname{pdim}\left(\mathbb{C}\left[X_{e, 1, n}\right]\right)=$ $\operatorname{pdim}\left(L_{1 / e}(\lambda)\right)+1=(e-2)\lfloor n / e\rfloor+1$. Since $\operatorname{dim}\left(X_{e, 1, n}\right)=n-e+1$, it follows that $\mathbb{C}\left[X_{e, 1, n}\right]$ is Cohen-Macaulay if and only if $e=2$ or $\lfloor n / e\rfloor=1$. This way, we recover part of [22, Proposition 3.11].

Example 8.5 Consider $e=4, n=10$. The minimal submodule in $\Delta_{1 / e}$ (triv) is $I_{4,1,10}$, and it is isomorphic to $L_{1 / e}\left(3^{3}, 1\right)$. Note that $c_{\lambda}=23 / 4$. The resolution of $L_{1 / 4}\left(3^{3}, 1\right)[-23 / 4]$ is given by

$$
\begin{aligned}
0 & \rightarrow\left(2,1^{8}\right)[-8] \rightarrow\left(2^{2}, 1^{6}\right)[-6] \rightarrow\left(2^{5}\right)[-3] \oplus\left(3^{2}, 1^{4}\right)[-2] \\
& \rightarrow\left(3^{2}, 2,1^{2}\right)[-1] \rightarrow\left(3^{3}, 1\right) \rightarrow L_{1 / 4}\left(3^{3}, 1\right)[-23 / 4] \rightarrow 0
\end{aligned}
$$

A resolution of the coordinate ring $\mathbb{C}\left[x_{1}, \ldots, x_{10}\right] / I_{4,1,10}$ looks similar, but each term is further shifted by -12 (because $c_{\text {triv }}-c_{\lambda}=-12$ ), and the end of the sequence is $\left(3^{3}, 1\right)[-12] \rightarrow(10) \rightarrow \mathbb{C}[\underline{x}] / I_{4,1,10} \rightarrow 0$. Note that the regularity of $\mathbb{C}\left[x_{1}, \ldots, x_{10}\right] / I_{4,1,10}$ is 15 .

Let us now compute the regularity of the subspace arrangement $X_{e, 1, n}$.

Proposition 8.6 The regularity of the $\mathbb{C}[\underline{x}]$-module $\mathbb{C}\left[X_{e, 1, n}\right]$ is given by

$$
\operatorname{reg}\left(\mathbb{C}\left[X_{e, 1, n}\right]\right)= \begin{cases}\lfloor n / e\rfloor(n-e+1)-1, & \text { if } n / e \in \mathbb{Z} \\ \lfloor n / e\rfloor(n-e+2)-1, & \text { else }\end{cases}
$$

Proof Let us write $n=(e-1) p+q=e p_{1}+q_{1}$, with $0 \leq q<e-1$ and $0 \leq q_{1}<e$. As above, let $\lambda=\left((e-1)^{p}, q\right)$ be the partition such that $L_{1 / e}(\lambda)$ is isomorphic to the socle of $\Delta_{1 / e}$ (triv). Note that the $e$-core of any partition in the block of triv $=(n)$ is $\left(q_{1}\right)$ and the $e$-weight is $p_{1}$. It then follows from the rimhook description of homological degree in Sect. 5.6 that the partition $\mu_{0}$ with highest homological degree in $\operatorname{Po}_{e}(\lambda)$ is given by adding $p_{1}$ vertical strips of length $e$ in the leftmost column to the $e$-core of $\lambda$ : thus $\mu_{0}=\left(a, 1^{n-a}\right)$ where $a=q_{1}$ if $q_{1}>0$ and $a=1$ if $q_{1}=0$, and $\operatorname{hd}\left(\mu_{0}\right)=(e-2) p_{1}$. Now it follows by a direct computation that

$$
\operatorname{reg}\left(\mathbb{C}\left[X_{e, 1, n}\right]\right)=c_{\mu_{0}}-c_{\text {triv }}-(e-2) p_{1}-1=\frac{(n-a) n}{e}-(e-2) p_{1}-1
$$


which coincides with the formula in the statement of the proposition.

\subsubsection{More BGG resolutions and a generalisation of the $e$-equals ideal.}

We take $\ell$ th powers and obtain a generalisation of the $e$-equals ideal. These subspaces arrangements admit commutative algebra resolutions which can be constructed via BGG-resolutions for the Cherednik algebra of $G(\ell, 1, n)$ (which we also construct in this section). Consider the ideal $I_{e, 1, n}(\ell)$ of polynomials vanishing on the set

$$
X_{e, 1, n}(\ell):=\mathfrak{S}_{n}\left\{\left(z_{1}, \ldots, z_{n}\right) \in \mathbb{C}^{n}: z_{1}^{\ell}=z_{2}^{\ell}=\cdots=z_{e}^{\ell}\right\} .
$$

Note that $X_{e, 1, n}(\ell)$ is an arrangement of $\ell^{e}\left(\begin{array}{l}n \\ e\end{array}\right)$ linear subspaces of $\mathbb{C}^{n}$, each of dimension $n-e+1$. When $e=\ell=2, X_{2,1, n}(2)$ is the braid arrangement of type $D_{n}$, consisting of reflection hyperplanes for the reflection representation of the Weyl group of type $D_{n}$ on $\mathbb{C}^{n}$. To give a set of generators for the ideal $I_{e, 1, n}(\ell)$, recall from the previous subsection the partition $\lambda=\left((e-1)^{p}, q\right)$ and the polynomial $p_{\lambda^{T}} \in \mathbb{C}\left[x_{1}, \ldots, x_{n}\right]$. According to [24, Proposition 2.5], a set of generators of the ideal $I_{e, 1, n}(\ell)$ is given by the $\mathfrak{S}_{n}$-images of $p_{\lambda^{T}}\left(x_{1}^{\ell}, \ldots, x_{n}^{\ell}\right)$.

Our next goal is to construct a graded-free resolution of the algebra of functions $\mathbb{C}\left[x_{1}, \ldots, x_{n}\right] / I_{e, 1, n}(\ell)$. In order to do this, we will use the following well-known commutative algebra result.

Lemma 8.7 Let $F_{1}, F_{2}, F_{3}$ be free $\mathbb{C}\left[x_{1}, \ldots, x_{n}\right]$-modules of finite rank, with bases $\left\{v_{1}^{1}, \ldots, v_{i_{1}}^{1}\right\},\left\{v_{1}^{2}, \ldots, v_{i_{2}}^{2}\right\}$ and $\left\{v_{1}^{3}, \ldots, v_{i_{3}}^{3}\right\}$, respectively. Let $A: F_{1} \rightarrow F_{2}$, $B: F_{2} \rightarrow F_{3}$ be morphisms defined in the given bases by matrices $\left(f_{i j}\left(x_{1}, \ldots, x_{n}\right)\right)$, $\left(g_{j k}\left(x_{1}, \ldots, x_{n}\right)\right)$, respectively, and define new morphisms $\widetilde{A}, \widetilde{B}$ by the matrices $\left(f_{i j}\left(x_{1}^{\ell}, \ldots, x_{n}^{\ell}\right)\right),\left(g_{j k}\left(x_{1}^{\ell}, \ldots, x_{n}^{\ell}\right)\right)$, respectively. If $\operatorname{im}(A)=\operatorname{ker}(B)$, then $\operatorname{im}(\widetilde{A})=$ $\operatorname{ker}(\widetilde{B})$.

Note that, for $\mu \in \mathscr{P}_{n}^{1}$, the module $\mathbb{C}\left[x_{1}, \ldots, x_{n}\right] \otimes S_{n}(\mu)$ has a distinguished basis indexed by $\operatorname{Std}(\mu)$. Thus, if $\lambda$ is a unitary partition, we can apply Lemma 8.7 to the complex $C_{\bullet}(\lambda)$ (viewed as a complex of free $\mathbb{C}\left[x_{1}, \ldots, x_{n}\right]$-modules) to obtain a complex $\widetilde{C}_{\bullet}(\lambda)$, which is exact outside of degree 0 . By construction, thanks to [24, Proposition 2.5], when $\lambda=\left((e-1)^{p}, q\right)$, the zeroth homology of $\widetilde{C}_{\bullet}(\lambda)$ coincides with the ideal $I_{e, 1, \lambda}(\ell)$. Moreover, by multiplying the grading shifts of $C_{\bullet}(\lambda)$ by $\ell$, this obtains a minimal graded-free resolution of $I_{e, 1, \lambda}$, and extending by $\mathbb{C}\left[x_{1}, \ldots, x_{n}\right]$, of the algebra of functions $\mathbb{C}\left[X_{e, 1, n}(\ell)\right]$. We then obtain the following result.

Proposition 8.8 The projective dimension of $\mathbb{C}\left[X_{e, 1, n}(\ell)\right]$ coincides with that of $\mathbb{C}\left[X_{e, 1, n}\right]$, which is $(e-2)\lfloor n / e\rfloor+1$ so that, regardless of $\ell, \mathbb{C}\left[X_{e, 1, n}(\ell)\right]$ is CohenMacaulay if and only if $e=2$ or $\lfloor n / e\rfloor=1$. The regularity of $\mathbb{C}\left[X_{e, 1, n}(\ell)\right]$ is given by

$$
\operatorname{reg}\left(\mathbb{C}\left[X_{e, 1, n}(\ell)\right]\right)= \begin{cases}\lfloor n / e\rfloor(\ell(n-1)-e+2)-1 & \text { if } n / e \in \mathbb{Z} \\ \lfloor n / e\rfloor(\ell n-e+2)-1 & \text { else }\end{cases}
$$


We have obtained the complex $\widetilde{C}_{\bullet}(\lambda)$ by means of pure commutative algebra. As it turns out, $\widetilde{C}_{\bullet}(\lambda)$ is a complex of standard modules for the rational Cherednik algebra of the group $G(\ell, 1, n):=\mathfrak{S}_{n} \ltimes(\mathbb{Z} / \ell \mathbb{Z})^{n}$ under a special class of parameters. The group $G(\ell, 1, n)$ is a complex reflection group, acting naturally on $\mathbb{C}^{n}$, and the rational Cherednik algebra depends on a function $\widetilde{c}: S \rightarrow \mathbb{C}$, where $S \subseteq G(\ell, 1, n)$ is the set of reflections and $\widetilde{c}(s)=\widetilde{c}\left(w s w^{-1}\right)$ for every $s \in S, w \in G(\ell, 1, n)$. Here, for a complex number $c \in \mathbb{C}$, we will take any function $\widetilde{c}$ such that $\widetilde{c}(s)=c$, if $s \in G(\ell, 1, n)$ is conjugate to a reflection in $\mathfrak{S}_{n}$. Any other reflection in $G(\ell, 1, n)$ is conjugate to a nonzero element of, say, the first copy of $\mathbb{Z} / \ell \mathbb{Z}$, so we have $\ell-1$ more parameters for $H_{\widetilde{c}}(G(\ell, 1, n))$, let us call them $c_{1}, \ldots, c_{\ell-1}$.

The rational Cherednik algebra $H_{\widetilde{c}}(G(\ell, 1, n))$ admits a presentation very similar to that of the rational Cherednik algebra $H_{c}\left(\mathfrak{S}_{n}\right)$ of the symmetric group. We will not give this presentation. Instead, we remark that $H_{\widetilde{c}}(G(\ell, 1, n))$ is the subalgebra of $\operatorname{End}_{\mathbb{C}}\left(\mathbb{C}\left[x_{1}, \ldots, x_{n}\right]\right)$ generated by the functions $x_{i}$ of multiplication by $x_{i}$ $(i=1, \ldots, n)$, the elements of $G(\ell, 1, n)$ (naturally viewed as automorphisms of $\left.\mathbb{C}\left[x_{1}, \ldots, x_{n}\right]\right)$ and the Dunkl-Opdam operators:

$$
\widetilde{D}_{i}:=\partial_{i}-c \sum_{j \neq i} \sum_{t=0}^{\ell-1} \frac{1}{x_{i}-\xi^{t} x_{j}}\left(1-(i j)^{t}\right)-\sum_{k=1}^{\ell-1} \frac{2 c_{k}}{\left(1-\xi^{-k}\right) x_{i}}\left(1-\xi_{i}^{k}\right)
$$

where $\xi:=\exp (2 \pi \sqrt{-1} / \ell), \xi_{i} \in G(\ell, 1, n)$ is the element that acts by multiplication by $\xi$ on the $i$-th coordinate in $\mathbb{C}^{n}$, and $(i j)^{t} \in G(\ell, 1, n)$ is $(i j)^{t}=\xi_{i}^{t} \xi_{j}^{-t}(i j)$. Let us remark that a similar presentation exists for the algebra $H_{c}\left(\mathfrak{S}_{n}\right)$, the Dunkl operators are now given by

$$
D_{i}=\partial_{i}-c \sum_{j \neq i} \frac{1}{x_{i}-x_{j}}(1-(i j))
$$

We will need the following result, that relates the operators $D_{i}$ and $\widetilde{D}_{i}$.

Lemma 8.9 For $g \in \mathbb{C}\left[x_{1}, \ldots, x_{n}\right]$, denote by $\widetilde{g}:=g\left(x_{1}^{\ell}, \ldots, x_{n}^{\ell}\right)$. Then, for any $i=1, \ldots, n$ :

$$
\widetilde{D}_{i}(\widetilde{g})=\ell x_{i}^{\ell-1} \widetilde{D_{i}(g)}
$$

Proof First of all, note that $\widetilde{g}$ is invariant under the action of $(\mathbb{Z} / \ell \mathbb{Z})^{n}$ on $\mathbb{C}\left[x_{1}, \ldots, x_{n}\right]$, and so it follows that 


$$
\widetilde{D}_{i}(\widetilde{g})=\partial_{i}(\widetilde{g})-c \sum_{j \neq i} \sum_{t=0}^{\ell-1} \frac{\widetilde{g}-(i j) \widetilde{g}}{x_{i}-\xi^{t} x_{j}} .
$$

Now let $h\left(x_{1}, \ldots, x_{n}\right) \in \mathbb{C}\left[x_{1}, \ldots, x_{n}\right]$ be such that $g-(i j) g=\left(x_{i}-x_{j}\right) h$. Note that it follows that $\widetilde{g}-(i j) \widetilde{g}=\left(x_{i}^{\ell}-x_{j}^{\ell}\right) \widetilde{h}$, so

$$
\sum_{t=0}^{\ell-1} \frac{\widetilde{g}-(i j) \tilde{g}}{x_{i}-\xi^{t} x_{j}}=\sum_{t=0}^{\ell} \prod_{\substack{k=0 \\ k \neq t}}^{\ell-1}\left(x_{i}-\xi^{k} x_{j}\right) \tilde{h}=\ell x_{i}^{\ell-1} \widetilde{h}
$$

and the result follows.

The algebra $H_{\widetilde{c}}(G(\ell, 1, n))$ still admits a triangular decomposition $H_{\widetilde{c}}(G(\ell, 1, n))$ $=\mathbb{C}\left[x_{1}, \ldots, x_{n}\right] \otimes \mathbb{C} G(\ell, 1, n) \otimes \mathbb{C}\left[y_{1}, \ldots, y_{n}\right]$, where $y_{i}$ is the Dunkl-Opdam operator $\widetilde{D}_{i}$. In particular, one can still define standard modules. For an irreducible representation $E$ of $G(\ell, 1, n)$, we have the standard module $\Delta_{\widetilde{c}}(E)$. As a $\mathbb{C}\left[x_{1}, \ldots, x_{n}\right]$-module, $\Delta_{\widetilde{c}}(E)=\mathbb{C}\left[x_{1}, \ldots, x_{n}\right] \otimes E$.

The irreducible representations, $S_{n}(\lambda)$, of $G(\ell, 1, n)$ are indexed by the set $\mathscr{P}_{n}^{\ell}$, and each $S_{n}(\lambda)$ has a natural basis indexed by the set $\operatorname{Std}(\lambda)$. In particular, if $\lambda \in \mathscr{P}_{n}^{1}$, we can consider the $\ell$-partition $\tilde{\lambda} \in \mathscr{P}_{n}^{\ell}$ given by $\tilde{\lambda}=(\lambda, \emptyset, \ldots, \emptyset)$. The $\operatorname{sets} \operatorname{Std}(\tilde{\lambda})$ and $\operatorname{Std}(\lambda)$ are obviously identified. Moreover, $G(\ell, 1, n)$ admits a natural surjection to $\mathfrak{S}_{n}$, and the irreducible representation $S_{n}(\widetilde{\lambda})$ of $G(\ell, 1, n)$ is simply given by the $\mathfrak{S}_{n}$-irreducible $S_{n}(\lambda)$ under this surjection.

Proposition 8.10 Let $c \in \mathbb{C}$. Then, for any $\lambda, \mu \in \mathscr{P}_{n}^{1}$ and any parameter $\tilde{c}$ as above, there is a natural identification

$$
\sim: \operatorname{Hom}_{H_{c}\left(\mathfrak{S}_{n}\right)}\left(\Delta_{c}(\lambda), \Delta_{c}(\mu)\right) \stackrel{\cong}{\longrightarrow} \operatorname{Hom}_{H_{\tilde{c}}(G(\ell, 1, n))}\left(\Delta_{\tilde{c}}(\tilde{\lambda}), \Delta_{\tilde{c}}(\tilde{\mu})\right)
$$

given as follows. For a standard Young tableau $\mathrm{t} \in \operatorname{Std}(\lambda)$, if $f \in \operatorname{Hom}_{H_{c}\left(\mathfrak{S}_{n}\right)}\left(\Delta_{c}(\lambda)\right.$, $\left.\Delta_{c}(\mu)\right)$ is given by $f(1 \otimes \mathrm{t})=\sum_{\mathrm{s} \in \operatorname{Std}(\mu)} f_{\mathrm{ts}}\left(x_{1}, \ldots, x_{n}\right) \otimes \mathrm{s}$, then $\tilde{f}(1 \otimes \mathrm{t})=$ $\sum_{\mathrm{s} \in \operatorname{Std}(\mu)} f_{\mathrm{ts}}\left(x_{1}^{\ell}, \ldots, x_{n}^{\ell}\right) \otimes \mathrm{s}$.

Proof We need to show, first, that $\left.\widetilde{f}\right|_{1 \otimes S_{n}(\widetilde{\lambda})}$ is a map of $G(\ell, 1, n)$-representations. This follows from the fact that, for any polynomial $g \in \mathbb{C}\left[x_{1}, \ldots, x_{n}\right], g\left(x_{1}^{\ell}, \ldots, x_{n}^{\ell}\right)$ is invariant under the action of $(\mathbb{Z} / \ell \mathbb{Z})^{n}$. Now we need to show that, for any standard Young tableau $\mathrm{t} \in \operatorname{Std}(\lambda), \widetilde{f}(1 \otimes \mathrm{t})$ is annihilated by all Dunkl operators $\widetilde{D}_{i}$. This is a direct consequence of Lemma 8.9.

This shows that $f \mapsto \tilde{f}$ does define a morphism, which is clearly injective. To show that it is bijective, let $h: \Delta_{\widetilde{c}}(\widetilde{\lambda}) \rightarrow \Delta_{\widetilde{c}}(\tilde{\mu})$ be a morphism. In particular, $\left.h\right|_{1 \otimes S_{n}(\tilde{\lambda})}$ is a map of $G(\ell, 1, n)$-modules. This implies that, if $h(1 \otimes \mathrm{t})=$ $\sum_{\mathrm{s} \in \operatorname{Std}(\mu)} h_{\mathrm{ts}}\left(x_{1}, \ldots, x_{n}\right) \otimes \mathrm{s}$, then $h_{\mathrm{ts}}\left(x_{1}, \ldots, x_{n}\right) \in \mathbb{C}\left[x_{1}^{\ell}, \ldots, x_{n}^{\ell}\right]$ for every $\mathrm{s} \in$ $\operatorname{Std}(\mu)$. Thanks to Lemma 8.9, this implies that $h=\tilde{f}$ for some $f: \Delta_{c}(\lambda) \rightarrow \Delta_{c}(\mu)$. 
Remark 8.11 If $c \notin 1 / 2+\mathbb{Z}$, then the existence of an isomorphism between $\operatorname{Hom}_{H_{c}\left(\mathfrak{S}_{n}\right)}\left(\Delta_{c}(\lambda), \Delta_{c}(\mu)\right)$ and $\operatorname{Hom}_{H_{\tilde{c}}(G(\ell, 1, n))}\left(\Delta_{\widetilde{c}}(\tilde{\lambda}), \Delta_{\widetilde{c}}(\tilde{\mu})\right)$ follows from [29, Proposition 5.9].

By Proposition 8.10 and Lemma 8.7, we have that if $\lambda$ is a unitary partition of $n$, then the complex $\widetilde{C}_{\bullet}(\lambda)$ is actually a complex of standard modules for $H_{\widetilde{c}}(G(\ell, 1, n))$, which is exact outside of degree zero, and thus it is a BGG resolution of its zeroth homology.

Remark 8.12 The zeroth homology of $\widetilde{C}_{\bullet}(\lambda)$ is not necessarily an irreducible $H_{\widetilde{c}}(G(\ell, 1, n))$-module. For example, if $\lambda=\left((e-1)^{p}, q\right)$, we have seen that $H_{0}\left(\widetilde{C}_{\bullet}(\lambda)\right)$ is the ideal $I_{e, 1, n}(\ell)$. When $\ell=2, e<n$ is even and the parameter $\widetilde{c}$ is such that $\widetilde{c}(s)=0$ if $s$ is not conjugate to a reflection in $\mathfrak{S}_{n}$, then this is an indecomposable, but not irreducible, $H_{\widetilde{c}}(G(\ell, 1, n))$-module.

Remark 8.13 Even if the zeroth homology of $\widetilde{C}_{\bullet}(\lambda)$ is irreducible (and thus it necessarily coincides with $\left.L_{\widetilde{c}}(\tilde{\lambda})\right)$ the natural Hermitian form on $L_{\widetilde{c}}(\widetilde{\lambda})$ does not need to be positive-definite, even if that for $L_{c}(\lambda)$ is. An example of this is given by taking $\ell=2$, odd $n, e=n, \lambda=(e-1,1)$ and the parameter $\widetilde{c}$ as in Remark 8.12. In this case, $L_{\widetilde{c}}(\widetilde{\lambda})=I_{e, 1, n}(2)$, which does not admit an invariant positive-definite Hermitian form, cf. [24, Proposition 7.1]

\subsubsection{The $(k, e)$-equals ideal}

We now consider the subspace arrangements of $k$ distinct clusters of $e$ equal parameters for $n=k e$. We show that the BGG resolution of $L$ (triv) is a minimal resolution of the coordinate ring of this subspace arrangement and generalise this to type $G(\ell, 1, n)$ as before.

Let $n=k e$, as we have seen, in this case we can give a BGG resolution of $L_{1 / e}$ (triv). It follows from [23, Theorem 5.10] that $\operatorname{rad}\left(\Delta_{1 / e}(\operatorname{triv})\right)$ is the ideal $I_{e, k, n}$ of functions vanishing on

$$
\begin{aligned}
X_{e, k, n} & :=\mathfrak{S}_{n}\left\{\left(z_{1}, \ldots, z_{n}\right) \in \mathbb{C}^{n}: z_{1}=\cdots=z_{e}, z_{e+1}\right. \\
& \left.=\cdots=z_{2 e}, \ldots, z_{(k-1) e+1}=\cdots=z_{k e}\right\} .
\end{aligned}
$$

Recall that the resolution of $L_{1 / e}$ (triv) is obtained as the Ringel dual of the resolution of $L_{1 / e}\left(e^{k}\right)$. Thus, the projective dimension of the algebra of functions $\mathbb{C}\left[X_{e, k, n}\right]=$ $\mathbb{C}[\underline{x}] / I_{e, k, n} \cong L_{1 / e}\left(\right.$ triv) $\left[c_{\text {triv }}\right]$ is $(e-1) k$. By the Auslander-Buchsbaum formula, the depth of $\mathbb{C}\left[X_{e, k, n}\right]$ is $n-(e-1) k=k$. So $\mathbb{C}\left[X_{e, k, n}\right]$ is always Cohen-Macaulay, and we recover a special case of [22, Proposition 3.11].

Let us now analyze the regularity of $L_{1 / e}\left(\right.$ triv) $\left[c_{\text {triv }}\right]$. By an argument similar to the proof of Proposition 8.6, this is given by $c_{\left(k^{e}\right)}-c_{\text {triv }}-(e-1) k$. By a direct computation, this is

$$
\operatorname{reg}\left(L_{1 / e}(\operatorname{triv})\left[c_{\text {triv }}\right]\right)=\frac{k(n-e-k+1)}{2}
$$


Example 8.14 Assume $e=3, n=6$. Then we have that a resolution of $L($ triv $)\left[c_{\text {triv }}\right]=$ $\mathbb{C}\left[x_{1}, \cdots, x_{6}\right] / I_{3,2,6}$ is given by

$$
\begin{aligned}
0 & \rightarrow\left(2^{3}\right)[-6] \rightarrow(3,2,1)[-5] \rightarrow\left(3^{2}\right)[-4] \oplus\left(4,1^{2}\right)[-4] \\
& \rightarrow(5,1)[-2] \rightarrow(6) \rightarrow \mathbb{C}[\underline{x}] / I_{3,2,6} \rightarrow 0
\end{aligned}
$$

and $\operatorname{reg}\left(\mathbb{C}[\underline{x}] / I_{3,2,6}\right)=2$.

Of course, for $\ell \geq 1$ we also have the subspace arrangement

$$
X_{e, k, n}(\ell):=\mathfrak{S}_{n}\left\{\left(z_{1}, \ldots, z_{n}\right) \in \mathbb{C}^{n}: z_{1}^{\ell}=\cdots=z_{e}^{\ell}, z_{e+1}^{\ell}=\cdots=z_{2 e}^{\ell}, \ldots, z_{(k-1) e+1}^{\ell}=\cdots=z_{k e}^{\ell}\right\}
$$

And its defining ideal $I_{e, k, n}(\ell)$. Since $I_{e, k, n}$ is the unique maximal submodule in $\Delta_{1 / e}$ (triv) and the submodules of this standard module are linearly ordered, the ideal $I_{e, k, n}$ is generated in a single degree. Thus, the exact same argument as that in the proof of [24, Proposition 2.5], if $q_{1}\left(x_{1}, \ldots, x_{n}\right), \ldots, q_{t}\left(x_{1}, \ldots, x_{n}\right)$ are generators of $I_{e, k, n}$ of minimal degree, then $q_{1}\left(x_{1}^{\ell}, \ldots, x_{n}^{\ell}\right), \ldots, q_{t}\left(x_{1}^{\ell}, \ldots, x_{n}^{\ell}\right)$ are generators of $I_{e, k, n}(\ell)$. It follows that the complex $\widetilde{C}_{\bullet}$ (triv) is a minimal graded-free resolution of the algebra of functions $\mathbb{C}\left[X_{e, k, n}(\ell)\right]$, and the variety $X_{e, k, n}(\ell)$ is always CohenMacaulay. Moreover, the regularity of $\mathbb{C}\left[X_{e, k, n}(\ell)\right]$ is given by $\ell\left(c_{\left(k^{e}\right)}-c_{\text {triv }}\right)-(e-1) k$, or more explicitly,

$$
\operatorname{reg}\left(\mathbb{C}\left[X_{e, k, n}(\ell)\right]\right)=\frac{k[\ell(n+e-k-1)-2(e-1)]}{2} .
$$

We remark that in general as $H_{\widetilde{c}}(G(\ell, 1, n))$-modules, $\mathbb{C}\left[X_{e, k, n}(n)\right]$ does not coincide with $L_{\widetilde{c}}$ (triv). For example, if $\ell=2, e=n$ is even and $\widetilde{c}(s)=0$ for a reflection $s$ not conjugate to an element of $\mathfrak{S}_{n}$, then $L_{\widetilde{1} / e}($ triv) is finite-dimensional, while $\mathbb{C}\left[X_{e, 1, n}(2)\right]$ is not.

Remark 8.15 Changing the parameter of the rational Cherednik algebra to $c=a / e>0$ with $\operatorname{gcd}(a ; e)=1$ does not change the shape of the resolution $C_{\bullet}(\lambda)$, so the projective dimension and depth of $L_{a / e}(\lambda)$ are independent of $a \in \mathbb{Z}_{>0}$ when $\lambda$ is $e$-unitary. However, the value of $c_{\lambda}$ is not independent of $a \in \mathbb{Z}_{>0}$, and we get

$$
\beta_{i, j}\left(L_{a / e}(\lambda)=\beta_{i, j / a}\left(L_{1 / e}(\lambda)\right)\right.
$$

where we implicitly agree that $\beta_{i, j / a}=0$ if $j / a \notin \mathbb{Z}$. For any such $a \in \mathbb{Z}_{>0}$ the module $L_{a / e}\left((m-1)^{p}, q\right)$ can be identified with an ideal of $\mathbb{C}[\underline{x}]$ whose vanishing set coincides with $X_{e, 1, n}$. This ideal is radical if and only if $a=1$, cf. [23, Theorem 5.10]. Similar considerations apply to $L_{a / e}$ (triv).

Acknowledgements E. Norton was supported by the Max Planck Institute for Mathematics, and J. Simental thanks the Hausdorff Institute for Mathematics where he was a participant at the Junior Trimester Program on Symplectic geometry and Representation theory. C. Bowman and E. Norton thank the organizers of the conference "Representation theory of symmetric groups and related algebras" held in December 2017 at the National University of Singapore, for providing an excellent venue for the fruitful exchange of mathematical 
ideas. The first author was funded by EPSRC fellowship grant EP/V00090X/1. We thank J. Chuang, G. Dobrovolska, S. Fishel, S. Griffeth, H. Ko, Z. Lin, P. Shan, C. Stroppel, and J. Torres for stimulating conversations and to further thank $\mathrm{S}$. Griffeth for his careful reading of an earlier version of this manuscript. We also thank the anonymous referee for their careful reading and many suggestions that improved the readability of this paper. S. Fishel and S. Griffeth have an alternative approach to [65, Conjecture 4.5]; our work was independent of each other. E. Norton dedicates this paper to "the Crew:" Danny, Jesse, and Joe.

Funding Open Access funding enabled and organized by Projekt DEAL.

Open Access This article is licensed under a Creative Commons Attribution 4.0 International License, which permits use, sharing, adaptation, distribution and reproduction in any medium or format, as long as you give appropriate credit to the original author(s) and the source, provide a link to the Creative Commons licence, and indicate if changes were made. The images or other third party material in this article are included in the article's Creative Commons licence, unless indicated otherwise in a credit line to the material. If material is not included in the article's Creative Commons licence and your intended use is not permitted by statutory regulation or exceeds the permitted use, you will need to obtain permission directly from the copyright holder. To view a copy of this licence, visit http://creativecommons.org/licenses/by/4.0/.

\section{References}

1. Bernstein, I.N., Gelfand, I.M., Gelfand, S.I.: Differential operators on the base affine space and a study of $\mathfrak{g}$-modules, 21-64

2. Bessenrodt, C., Olsson, J.B.: On residue symbols and the Mullineux conjecture. J. Algebraic Combin. 7, 227-251 (1998)

3. Bezrukavnikov, R., Etingof, P.: Parabolic induction and restriction functors for rational Cherednik algebras. Selecta Math. (N.S.) 14, 397-425 (2009)

4. Boe, B., Hunziker, M.: Kostant modules in blocks of category $\mathscr{O}_{S}$. Commun. Algebra 37, 323-356 (2009)

5. Bowman, C.: The many graded cellular bases of Hecke algebras, to appear in Amer. J. Math. arXiv: 1702.06579

6. Bowman, C., Cox, A.G.: Modular decomposition numbers of cyclotomic Hecke and diagrammatic Cherednik algebras: A path theoretic approach. Forum Math. Sigma 6, (2018)

7. Bowman, C., Cox, A., Speyer, L.: A family of graded decomposition numbers for diagrammatic Cherednik algebras. Int. Math. Res. Not. IMRN (2017). https://doi.org/10.1093/imrn/rnw101

8. Bowman, C., Speyer, L.: Kleshchev's decomposition numbers for diagrammatic Cherednik algebras. Trans. Am. Math. Soc. 370, 3551-3590 (2018)

9. Braden, T.: Perverse sheaves on Grassmannians. Can. J. Math. 54, 493-532 (2002)

10. Brundan, J., Kleshchev, A.: Blocks of cyclotomic Hecke algebras and Khovanov-Lauda algebras. Invent. Math. 178, 451-484 (2009)

11. Brundan, J., Stroppel, C.: Highest weight categories arising from Khovanov's diagram algebra. II. Koszulity. Transform. Groups 15(1), 1-45 (2010)

12. Brundan, J., Stroppel, C.: Highest weight categories arising from Khovanov's diagram algebra III: category $\mathcal{O}$. Represent. Theory 15, 170-243 (2011)

13. Cherednik, I.: Affine Hecke algebras via DAHA. Arnold Math. J. 4, 69-85 (2018)

14. Chuang, J., Miyachi, H.: Runner removal Morita equivalences. In: A. Gyoja, H. Nakajima, K. Shinoda, T. Shoji, T. Tanisaki. (eds.) Representation theory of algebraic groups and quantum groups. Progr. Math., vol. 284, Birkhäuser/Springer, New York, pp.55-79 (2010)

15. Ciubotaru, D.: Dirac cohomology for symplectic reflection algebras. Selecta Math. (N.S.) 22, 111-144 (2016)

16. Derksen, H., Sidman, J.: A sharp bound for the Castelnuovo-Mumford regularity of subspace arrangements. Adv. Math. 172(2), 151-157 (2002)

17. Dipper, R., James, G., Mathas, A.: Cyclotomic $q$-Schur algebras. Math. Z. 229, 385-416 (1998)

18. Dunkl, C.F., de Jeu, M.F.E., Opdam, E.M.: Singular polynomials for finite reflection groups. Trans. Am. Math. Soc. 346, 237-256 (1994)

19. Eastwood, M.: Higher symmetries of the Laplacian. Ann. Math. 161, 1645-1665 (2005) 
20. Enright, T.J., Hunziker, M.: Resolutions and Hilbert series of determinantal varieties and unitary highest weight modules. J. Algebra 273, 608-639 (2004)

21. Etingof, P., Ginzburg, V.: Symplectic reflection algebras, Calogero-Moser space, and deformed HarishChandra homomorphism. Invent. Math. 147, 243-348 (2002)

22. Etingof, P., Gorsky, E., Losev, I.: Representations of rational Cherednik algebras with minimal support and torus knots. Adv. Math. 277, 124-180 (2015)

23. Etingof, P., Stoica, E.: Unitary representations of rational Cherednik algebras. Represent. Theory 13, 349-370 (2009). (With an appendix by Stephen Griffeth)

24. Feigin, M., Shramov, C.: On unitary submodules in the polynomial representations of rational Cherednik algebras. Int. Math. Res. Not IMRN 2012, 3375-3414 (2012)

25. Foda, O., Leclerc, B., Okado, M., Thibon, J., Welsh, T.: Branching functions of $A_{n-1}^{(1)}$ and Jantzen-Seitz problem for Ariki-Koike algebras. Adv. Math. 141, 322-365 (1999)

26. Ford, B., Kleshchev, A.: A proof of the Mullineux conjecture. Math. Z. 226(2), 267-308 (1997)

27. Gainutdinov, A., Jacobsen, J., Saleur, H., Vasseur, R.: A physical approach to the classification of indecomposable Virasoro representations from the blob algebra. Nuclear Phys. B 873, 614-681 (2013). https://doi.org/10.1016/j.nuclphysb.2013.04.017

28. Garland, H., Lepowsky, J.: Lie algebra homology and the Macdonald-Kac formulas. Invent. Math. 34, 37-76 (1976)

29. Ginzburg, V., Guay, N., Opdam, E., Rouquier, R.: On the category $\mathcal{O}$ for rational Cherednik algebras. Invent. Math. 154, 617-651 (2003). https://doi.org/10.1007/s00222-003-0313-8

30. Griffeth, S.: Unitary representations of cyclotomic rational Cherednik algebras. J. Algebra 512, 310356 (2018)

31. Harish-Chandra, et al.: Harmonic analysis on real reductive groups. I. The theory of the constant term. J. Functional Anal. 19, 104-204 (1975)

32. Harish-Chandra et al.: Harmonic analysis on real reductive groups. II. Invent. Math. 36, 1-55 (1976)

33. Harish-Chandra, et al.: Harmonic analysis on real reductive groups. III. The Maass-Selberg relations and the Plancherel formula. Ann. Math. (2) 104(1), 117-201 (1976)

34. Hu, J., Mathas, A.: Graded cellular bases for the cyclotomic Khovanov-Lauda-Rouquier algebras of type A. Adv. Math. (2010). https://doi.org/10.1016/j.aim.2010.03.002

35. Huang, J.-S., Pandžić, P., Vogan, D.: On classifying unitary modules by their Dirac cohomology. Sci. China Math. 60, 1937-1962 (2017)

36. Huang, J., Wong, K.: A Casselman-Osborne theorem for rational Cherednik algebras. Transform. Groups 23, 75-99 (2018)

37. Kleshchev, A.: Completely splittable representations of symmetric groups. J. Algebra 181, 584-592 (1996)

38. Kleshchev, A.S.: Branching rules for modular representations of symmetric groups. III. Some corollaries and a problem of Mullineux. J. London Math. Soc. (2) 54(1), 25-38 (1996)

39. Kostant, B.: Lie algebra cohomology and the generalized Borel-Weil theorem. Ann. Math. (2) 74, 329-387 (1961)

40. Lascoux, A.: Syzygies des variétés déterminantales, Adv. in Math. 30 (1978)

41. Leclerc, B., Thibon, J.-Y.: Littlewood-Richardson coefficients and Kazhdan-Lusztig polynomials, Combinatorial methods in representation theory (Kyoto, 1998). Adv. Stud. Pure Math. 28, 155-220 (2000)

42. Losev, I.: Derived equivalences for rational Cherednik algebras. Duke Math. J. 166, 27-73 (2017)

43. Losev, I., Shelley-Abrahamson, S.: On refined filtration by supports for rational Cherednik categories O. Selecta Math. (N.S.) 24(2), 1729-1804 (2018)

44. Lusztig, G., Williamson, G.: Billiards and tilting characters for $\mathrm{SL}_{3}$. SIGMA Integrability Geom. Methods Appl. 14, 015, 22 pages (2018)

45. Lusztig, G., Williamson, G.: On the character of certain tilting modules. Sci. China Math. 61, 295-298 (2018)

46. Martin, P., Saleur, H.: The blob algebra and the periodic Temperley-Lieb algebra. Lett. Math. Phys. (1994). https://doi.org/10.1007/BF00805852

47. Martin, P., Woodcock, D.: Generalized blob algebras and alcove geometry. LMS J. Comput. Math. (2003). https://doi.org/10.1112/S1461157000000450

48. Mullineux, G.: Bijections of $p$-regular partitions and $p$-modular irreducibles of the symmetric groups. J. London Math. Soc. (2) 20(1), 60-66 (1979) 
49. Ram, A.: Skew shape representations are irreducible. In: Kang, S-J., Lee, K-H. (eds.) Combinatorial and geometric representation theory (Seoul, 2001). Contemp. Math., vol. 325, pp. 161-189. Amer. Math. Soc. Providence, RI (2003)

50. Riche, S., Williamson, G.: Tilting modules and the $p$-canonical basis, arXiv:1512.08296, 2016, preprint

51. Rouquier, R.: $q$-Schur algebras and complex reflection groups, Mosc. Math. J. 8 (2008)

52. Rouquier, R., Shan, P., Varagnolo, M., Vasserot, E.: Categorifications and cyclotomic rational double affine Hecke algebras. Invent. Math. 204, 671-786 (2016). https://doi.org/10.1007/s00222-015-06237

53. Ruff, O.: Completely splittable representations of symmetric groups and affine Hecke algebras. J. Algebra 181, 584-592 (2006)

54. Stoica, E.: Unitary representations of hecke algebras of complex reflection groups, arxiv:0910.0680.pdf

55. Stroppel, C.: Parabolic category $\mathscr{O}$, perverse sheaves on Grassmannians, Springer fibres and Khovanov homology, Compos. Math. 145 (2009)

56. Stroppel, C., Webster, B.: Quiver Schur algebras and $q$-Fock space, arXiv:1110.1115, (2011), preprint

57. Teitler, Z., Torrance, D.A.: Castelnuovo-Mumford regularity and arithmetic Cohen-Macaulayness of complete bipartite subspace arrangements. J. Pure Appl. Algebra 219(6), 2134-2138 (2015)

58. Vogan, D.A. Jr.: A Langlands classification for unitary representations. In: Kobayashi, T., Kashiwara, M., Matsuki, T., Nishiyama, K., Oshima, T. (eds.) Analysis on homogeneous spaces and representation theory of Lie groups, Adv. Stud. Pure Math., vol. 26, pp. 299-324. Math. Soc. Japan, Tokyo (2000)

59. Webster, B.: Rouquier's conjecture and diagrammatic algebra. Forum Math. Sigma 5, e27, 71 (2017)

60. Webster, B.: Representation theory of the cyclotomic Cherednik algebra via the Dunkl-Opdam subalgebra New York. J. Math. 25, 1017-1047 (2019)

61. Wigner, E.: On unitary representations of the inhomogeneous Lorentz group. Ann. Math. (2) 40, 149-204 (1939)

62. Wilcox, S.: Supports of representations of the rational Cherednik algebra of type A. Adv. Math. 314, 426-492 (2017)

63. Williamson, G.: Schubert calculus and torsion explosion. J. Am. Math. Soc. 30, 1023-1046 (2017)

64. Yee, W.: Relating signed and classical Kazhdan-Lusztig polynomials. Duke Math. J. 163, 2161-2178 (2014)

65. Zamaere, C.B., Griffeth, S., Sam, S.V.: Jack polynomials as fractional quantum Hall states and the Betti numbers of the $(k+1)$-equals ideal. Commun. Math. Phys. 330, 415-434 (2014)

Publisher's Note Springer Nature remains neutral with regard to jurisdictional claims in published maps and institutional affiliations. 